Draft Version SEPTEMber 11, 2018

Preprint typeset using LATEX style emulateapj v. 06/18/13

\title{
BROAD ABSORPTION LINE VARIABILITY ON MULTI-YEAR TIMESCALES IN A LARGE QUASAR SAMPLE
}

\author{
N. Filiz Ak ${ }^{1,2,3}$, W. N. Brandt ${ }^{1,2}$, P. B. Hall ${ }^{4}$, D. P. Schneider ${ }^{1,2}$, S. F. Anderson ${ }^{5}$, F. Hamann $^{6}$, B. F. $^{2}$ \\ Lundgren $^{7,8}$, Adam D. Myers ${ }^{9}$, I. PÂris ${ }^{10}$, P. Petitjean ${ }^{11}$, Nicholas P. Ross ${ }^{12}$, Yue Shen ${ }^{13,14,15}$, Don York ${ }^{16,17}$ \\ Draft version September 11, 2018
}

\section{ABSTRACT}

We present a detailed investigation of the variability of 428 C IV and 235 Si IV Broad Absorption Line (BAL) troughs identified in multi-epoch observations of 291 quasars by the Sloan Digital Sky Survey-I/II/III. These observations primarily sample rest-frame timescales of 1-3.7 yr over which significant rearrangement of the BAL wind is expected. We derive a number of observational results on, e.g., the frequency of BAL variability, the velocity range over which BAL variability occurs, the primary observed form of BAL-trough variability, the dependence of BAL variability upon timescale, the frequency of BAL strengthening vs. weakening, correlations between BAL variability and BALtrough profiles, relations between C IV and Si IV BAL variability, coordinated multi-trough variability, and BAL variations as a function of quasar properties. We assess implications of these observational results for quasar winds. Our results support models where most BAL absorption is formed within an order-of-magnitude of the wind-launching radius, although a significant minority of BAL troughs may arise on larger scales. We estimate an average lifetime for a BAL trough along our line-of-sight of a few thousand years. BAL disappearance and emergence events appear to be extremes of general BAL variability, rather than being qualitatively distinct phenomena. We derive the parameters of a random-walk model for BAL EW variability, finding that this model can acceptably describe some key aspects of EW variability. The coordinated trough variability of BAL quasars with multiple troughs suggests that changes in "shielding gas" may play a significant role in driving general BAL variability.

\section{INTRODUCTION}

The high-velocity winds from quasars are important for several related reasons. First, these winds can significantly affect observed quasar properties via, e.g., ultraviolet (UV) line absorption, high-ionization line emission, optical/UV reddening, and X-ray ab-

nfilizak@astro.psu.edu

${ }^{1}$ Department of Astronomy \& Astrophysics, Pennsylvania State University, University Park, PA, 16802, USA

${ }_{2}$ Institute for Gravitation and the Cosmos, Pennsylvania State University, University Park, PA 16802, USA

${ }^{3}$ Faculty of Sciences, Department of Astronomy and Space Sciences, Erciyes University, 38039 Kayseri, Turkey

${ }^{4}$ Department of Physics and Astronomy, York University, 4700 Keele St., Toronto, Ontario, M3J 1P3, Canada

5 Astronomy Department, University of Washington, Seattle, WA 98195, USA

6 Department of Astronomy, University of Florida, Gainesville, FL 32611-2055, USA

7 Department of Astronomy, University of Wisconsin, Madison, WI 53706, USA

${ }^{8}$ NSF Astronomy and Astrophysics Postdoctoral Fellow

9 Department of Physics and Astronomy, University of Wyoming, Laramie, WY 82071, USA

10 Departamento de Astronomía, Universidad de Chile, Casilla 36-D, Santiago, Chile

${ }^{11}$ Universite Paris 6, Institut d'Astrophysique de Paris, 75014, Paris, France

12 Lawrence Berkeley National Laboratory, 1 Cyclotron Road, Berkeley, CA 92420, USA

13 Harvard-Smithsonian Center for Astrophysics, 60 Garden St., MS-51, Cambridge, MA 02138, USA

${ }^{14}$ Carnegie Observatories, 813 Santa Barbara Street, Pasadena, CA 91101, USA

15 Hubble Fellow

16 The University of Chicago, Department of Astronomy and Astrophysics, Chicago, IL 60637, USA

17 The University of Chicago, Enrico Fermi Institute, Chicago, IL 60637, USA sorption (e.g., Wevmann et al. 1981; Turnshek 1988; Leighly 2004; Collin et al. 2006; Gallagher et al. 2002, 2006; Gibson et al. 2009; Richards et al. 2011). Second, wind absorption lines are observed frequently, indicating that winds have a high covering factor and are a substantial part of quasar nuclear regions (e.g., Ganguly \& Brotherton 2008; Gibson et al. 2009; Allen et al. 2011). Third, winds might improve the efficiency of accretion onto the central supermassive black hole (SMBH) by removing angular momentum from the accretion disk (e.g., Emmering et al. 1992; Konigl \& Kartje 1994). Finally, winds can evacuate gas from the host galaxy, perhaps shaping SMBH growth and galaxy evolution (e.g., Di Matteo et al.|2005; Chartas et al. 2009; Rupke \& Veilleux 2011; Sturm et al. 2011; Borguet et al. 2013).

The strongest absorption lines created by quasar winds are Broad Absorption Line (BAL) troughs with velocity widths greater than $2000 \mathrm{~km} \mathrm{~s}^{-1}$ and typical outflow velocities of $1000-30000 \mathrm{~km} \mathrm{~s}^{-1}$ (e.g., Wevmann et al. 1991). Many BALs are believed to be formed in an equatorial wind that is launched from the accretion disk at 10-100 light days from the SMBH (e.g., Murray et al. 1995; Proga 2000). If the BALs are formed in the vicinity of the launching region, then the timescale for wind material to cross the region of interest is about $1-10 \mathrm{yr}$, and this is a reasonable characteristic timescale over which flow structures might be expected to change. This is also the characteristic timescale for significant angular rotation of the accretion disk at the wind-launching radius. Assessments of the transverse velocities of BAL material indicate these are often comparable to the aforementioned outflow velocities (e.g., Capellupo et al.|2011; 
Hall et al. 2011), and characteristic variability timescales of years are again deduced for material moving transversely through our line-of-sight. Thus, studies of multiyear BAL variability can provide useful insights into the nature of quasar winds.

The existence of BAL variability has been known for over two decades (e.g., Smith \& Penston 1988; Turnshek et al. 1988; Barlow et al. 1992). Early investigations of this phenomenon were generally singleobject studies with 2-4 observational epochs, although Barlow (1993) performed an early spectroscopic monitoring survey of $23 \mathrm{BAL}$ quasars. In recent years, systematic sample-based studies of BAL variability, investigating 5-30 objects, have become increasingly common (e.g., Lundgren et al. 2007; Gibson et al. 2008, 2010; Capellupo et al. 2011, 2012; Filiz Ak et al. 2012; Haggard et al. 2012; Miller et al. 2012; Vivek et al. 2012); see Table 1 for a summary of the basic properties of these samples. Sample-based studies have the advantage of allowing broadly applicable and statistically reliable conclusions about BAL variability to be drawn. BAL variability has been found to be a complex and diverse phenomenon. Generally, changes in the residual flux in portions of BAL troughs are observed, while detections of BAL acceleration/deceleration are much rarer (e.g., Vilkoviskii \& Irwin 2001; Gibson et al. 2008, 2010; Capellupo et al. 2012).

We have been using observations taken as part of the ongoing Baryon Oscillation Spectroscopic Survey (BOSS; Dawson et al. 2013) of the Sloan Digital Sky Survey-III (SDSS-III; Eisenstein et al. 2011) to perform the largest survey of multi-year BAL variability to date (see Section 2.1 and Filiz Ak et al. 2012 for further description). Our final sample will include $\approx 2100$ BAL quasars with high-quality spectra providing multi-year variability coverage in the rest frame; this size is about two orders of magnitude larger than other samples being used to investigate multi-year BAL variability (see Table 11). In Filiz Ak et al. (2012) we presented some first results from the survey focused on C IV BAL disappearance events. In this paper, we provide more general findings regarding the variability of $\mathrm{C}$ IV and Si IV BAL troughs, primarily on multi-year timescales but also extending to much shorter timescales. These findings are based upon 291 BAL quasars selected from our full sample to have particularly high-quality spectroscopic coverage of these troughs; 428 distinct C IV and 235 distinct Si IV troughs are utilized. Our overall approach is first to characterize systematically how BAL troughs vary on multi-year timescales and then to use this characterization to derive physical implications for BAL outflows. For example, our results provide insights into the radial distance where most BAL troughs are formed, the lifetimes of BAL troughs along our line of sight, the connection between BAL disappearance/emergence events and general BAL variability, and the driving mechanisms of BAL variability.

In Section 2 we describe the observations and sample selection underlying this work, and in Section 3] we describe data preparation and analysis approaches. Our observational results on BAL variability are presented in Section 4. In Section 5 we provide a discussion of implications for quasar winds, and in Section 6 we present a summary and describe promising future avenues of rele- vant research.

Throughout this work we use a cosmology with $H_{0}=70 \mathrm{~km} \mathrm{~s}^{-1} \mathrm{Mpc}^{-1}, \Omega_{M}=0.3$, and $\Omega_{\Lambda}=0.7$. All time intervals and EWs are in the rest frame of the quasar unless stated otherwise. Negative signs for velocities indicate that a BAL trough is blueshifted with respect to the systemic velocity. We define EWs of absorption features to be positive. Positive EW variations indicate strengthening, and negative values indicate weakening.

\section{OBSERVATIONS AND SAMPLE SELECTION}

\subsection{Observations}

We have utilized data from the Sloan Digital Sky Survey-I/II (hereafter "SDSS"; York et al. 2000) and BOSS that use a mosaic CCD camera Gunn et al. 1998) plus multi-object spectrograph on a dedicated $2.5 \mathrm{~m}$ telescope (Gunn et al. 2006) at Apache Point Observatory. Between 2000-2008, the SDSS I/II completed spectroscopy over $9380 \mathrm{deg}^{2}$ and obtained over 1.6 million spectra in total, including more than 105000 quasars (e.g., Richards et al. 2002; Abazajian et al. 2009; Schneider et al. 2010). BOSS is observing a sample of $\approx 210000$ quasars, the majority of which are at $z>2.2$ (Ross et al. 2012; Pâris et al. 2012), with the main scientific motivation being to measure the baryon acoustic oscillation (BAO) feature in the Lyman- $\alpha$ forest (e.g., Busca et al. 2013; Slosar et al. 2013). Using an improved spectrograph, BOSS spectra have coverage between 3600-10000 $\AA$ at a resolution of 1300-3000 (see Dawson et al. 2013; Smee et al. 2013).

In addition to its primary quasar program, BOSS is executing several ancillary projects (see Dawson et al. 2013) including one focused on investigating the dynamics of quasar winds over multi-year timescales. This project re-observes selected bright BAL quasars that have previous SDSS spectral observations to enable a high-quality and relatively unbiased study of BAL variability over multi-year timescales in the rest frame of the quasar. The project targets were selected using information from the catalog of BAL quasars for SDSS DR5 (Gibson et al. 2009) and the SDSS DR5 quasar catalog (Schneider et al. 2007). The details of the BAL target selection are described in Section 2.1 of Filiz Ak et al. (2012). Briefly, the 2005 selected targets are optically bright $(i<19.3)$ BAL quasars with redshifts $0.48 \leq z \leq 4.65$. The observed SDSS spectra of these targets have a signal-to-noise ratio per $0.4 \AA$ pixel at 1650-1750 $\AA$ of $\mathrm{SN}_{1700}>6 ; \mathrm{SN}_{1700}$ is defined for the continuum redward of C IV and should not be affected by BAL absorption. The targets were chosen to have a modified balnicity index $\mathrm{BI}_{0}>100 \mathrm{~km} \mathrm{~s}^{-1}$. $\mathrm{BI}_{0}$ is defined by Gibson et al. (2008) using the following equation:

$$
\mathrm{BI}_{0} \equiv \int_{0}^{-25000}\left(1-\frac{f(v)}{0.9}\right) C d v
$$

where $f(v)$ is the normalized flux density as a function of velocity, $v$, and $C$ is a constant which is equal to 1.0 only when a trough is wider than $2000 \mathrm{~km} \mathrm{~s}^{-1}$, it is otherwise 0.0 .

In this study, we use SDSS spectra observed between MJD 51602 and 54557 (2000 February 28 to 2008 January 04) and BOSS spectra observed between MJD 55176 
and 56109 (2009 December 11 to 2012 July 01); i.e., we utilize spectra taken after the completion of hardware commissioning for both SDSS and BOSS. Between these dates, 1087 of the 2005 targets from the ancillary project were observed by BOSS.

\subsection{Sample Selection}

In this section we present the selection criteria used to create a "main sample" for studying C IV and Si IV BAL variability on multi-year timescales in the rest frame. We select our main-sample BAL quasars from the targets observed via the ancillary project based on the following criteria:

1. To enable more robust continuum fits, we select only the quasars that have spectral coverage of the relatively line free (RLF, see Section 3.1) windows blueward of the Si IV line as well as redward of the $\mathrm{C}$ IV line; these windows play a key role in constraining the fitted continuum. Thus, we utilize the quasars that have $z>2$ from the sample of 1087 observed objects. (619 quasars)

2. The targeted sample of quasars was required to have $\mathrm{SN}_{1700}>6$ for the SDSS spectrum, although higher values of $\mathrm{SN}_{1700}$ are advantageous for the study of moderate or weak BAL variations. We have thus chosen to utilize quasars that have $\mathrm{SN}_{1700}>10$ for both the SDSS and BOSS spectra; visual inspection shows that this choice provides a good balance between high spectral quality and large sample size. (356 quasars out of 619)

3. As in Filiz Ak et al. (2012), to avoid confusion between emission-line and BAL variability, we consider only the BAL troughs that are significantly detached from the CIV and Si IV emission lines by setting velocity limits for BAL troughs. We consider the BAL regions of each transition that lie between -3000 and $-30000 \mathrm{~km} \mathrm{~s}^{-1}$. To select the quasars with moderate-to-strong BAL troughs, we require that the balnicity index of both the SDSS and BOSS spectra of the main-sample quasars have $\mathrm{BI}^{\prime}>100 \mathrm{~km} \mathrm{~s}^{-1}$; a consistent threshold for both SDSS and BOSS is required to avoid biases in our later analyses. $\mathrm{BI}^{\prime}$ is defined by Filiz Ak et al. (2012) using the following equation:

$$
\mathrm{BI}^{\prime} \equiv \int_{-3000}^{-30000}\left(1-\frac{f(v)}{0.9}\right) C d v .
$$

Similar to the $\mathrm{BI}_{0}$ definition, in this equation $f(v)$ is the normalized flux density as a function of velocity, $v$, and $C$ is a constant which is equal to 1.0 only when a trough is wider than $2000 \mathrm{~km} \mathrm{~s}^{-1}$, it is otherwise 0.0. (297 quasars out of 356)

4. We have rejected six quasars $(2 \%)$ from our main sample because of difficulties in defining the continua and/or emission lines in their spectra. Spectra of these quasars possess strong absorption lines of many transitions causing large systematic uncertainties in BAL measurements. (291 quasars out of 297)
Based on these criteria, we selected 291 BAL quasars as our main sample. All 291 quasars have at least one observation from SDSS and one from BOSS, and $22 \%$ have additional SDSS and/or BOSS observations. In total, our sample contains 699 spectra of main-sample quasars that cover rest-frame timescales from $5.9 \mathrm{hr}$ to $3.7 \mathrm{yr}$.

We have cross-matched our 291 main-sample quasars with the catalog of quasar properties from SDSS DR7 (Shen et al. 2011) and obtained their absolute $i$ band magnitudes, $M_{i}$, estimated bolometric luminosities, $L_{\mathrm{Bol}}$, Eddington-luminosity ratios, $L_{\mathrm{Bol}} / L_{\mathrm{Edd}}$, and virial black-hole mass estimates, $M_{\mathrm{BH}}$. In addition we use the radio-loudness parameter, $R$, defined as $R=$ $f_{6 \mathrm{~cm}} / f_{2500 \AA}$, where $f_{6 \mathrm{~cm}}$ is the radio flux density at restframe $6 \mathrm{~cm}$ and $f_{2500 \AA}$ is the optical flux density at restframe $2500 \AA$. Shen et al. (2011) calculated the $R$ parameter using radio emission detected in Very Large Array (VLA) Faint Images of the Radio Sky at TwentyCentimeters (FIRST; Becker et al. 1995) observations. We obtain redshift values from Hewett \& Wild (2010), and these are used throughout.

We illustrate some of the basic properties of our sample in Figures 1 and 2, Figure 1 shows $M_{i}$ vs. redshift for the main sample of this paper, the 2005 targets of the BOSS ancillary project on BAL quasars, and all SDSS DR5 BAL quasars. The $M_{i}$ distribution of the main-sample quasars spans about the same range as that of the general ancillary program targets from $z=2-4$, covering a factor of $\approx 5$ in luminosity at any given redshift. Figure 2 compares the $i$-band apparent magnitude distributions of our main sample, the 2005 BOSS ancillary project targets, and the BAL quasars identified in the SDSS DR10 quasar catalog (Pâris et al. 2013). It is clear that the main sample and ancillary project effectively cover the brightest BAL quasars that generally provide the highest quality SDSS and BOSS spectra. The mean $i$-band magnitude is 18.4 for the main-sample quasars and 18.7 for the ancillary project targets. Using the catalog of BAL quasars for SDSS DR5 (Gibson et al. 2009), we found that 22 of the 291 main-sample quasars possess Al III BALs and thus are identified as low-ionization BAL quasars. Additional unidentified low-ionization BAL quasars may be present; e.g., among objects that lack spectral coverage of the important Mg II low-ionization transition.

\section{DATA PREPARATION AND ANALYSIS}

\subsection{Basic Spectral Preparation}

For the purpose of investigating BAL variability, we compared the multi-epoch spectral observations of our main-sample quasars. We have normalized each spectrum by a model for the continuum following the procedure in Section 3.1 of Filiz Ak et al. (2012). Briefly, we first correct the spectra for Galactic extinction using the $A_{V}$ values from Schlegel et al. (1998) and then transform from the observed frame to the rest frame using the redshift values from Hewett \& Wild (2010). We remove the pixels from the spectra that contain significant night-sky line residuals that are flagged by the SDSS and BOSS data-reduction pipelines.

To reconstruct the underlying continuum, as in Gibson et al. (2008, 2009) and Filiz Ak et al. (2012), we define RLF windows to be the following spectral regions: $1250-1350 \AA, 1700-1800 \AA, 1950-2200 \AA$, 


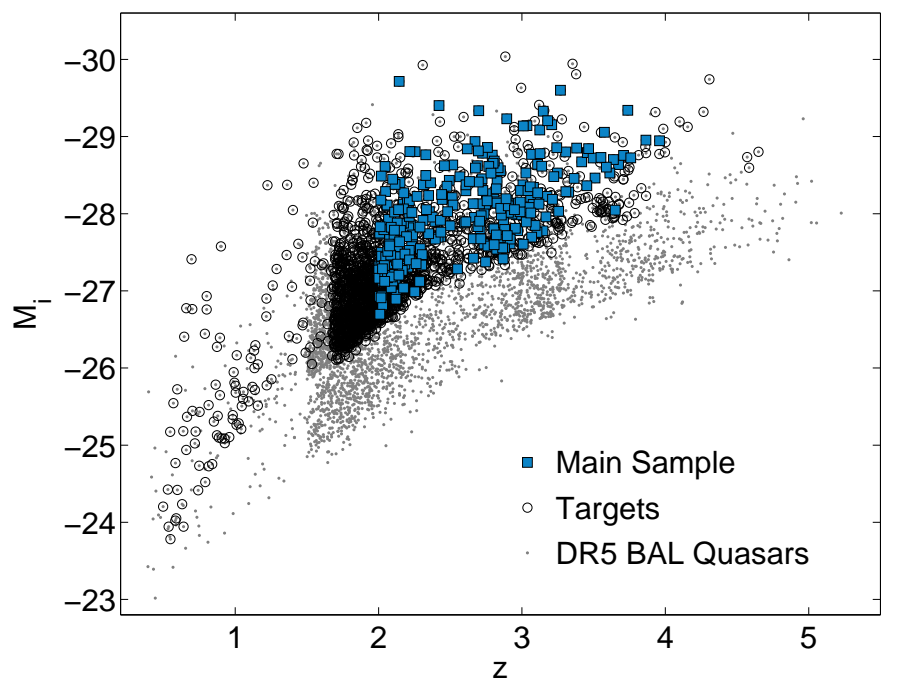

FIG. 1.- Absolute $i$-band magnitude, $M_{i}$, vs. redshift for the main sample of this paper (blue squares), the 2005 targets of the BOSS ancillary project on BAL quasars (open circles), and all SDSS DR5 BAL quasars (dots).

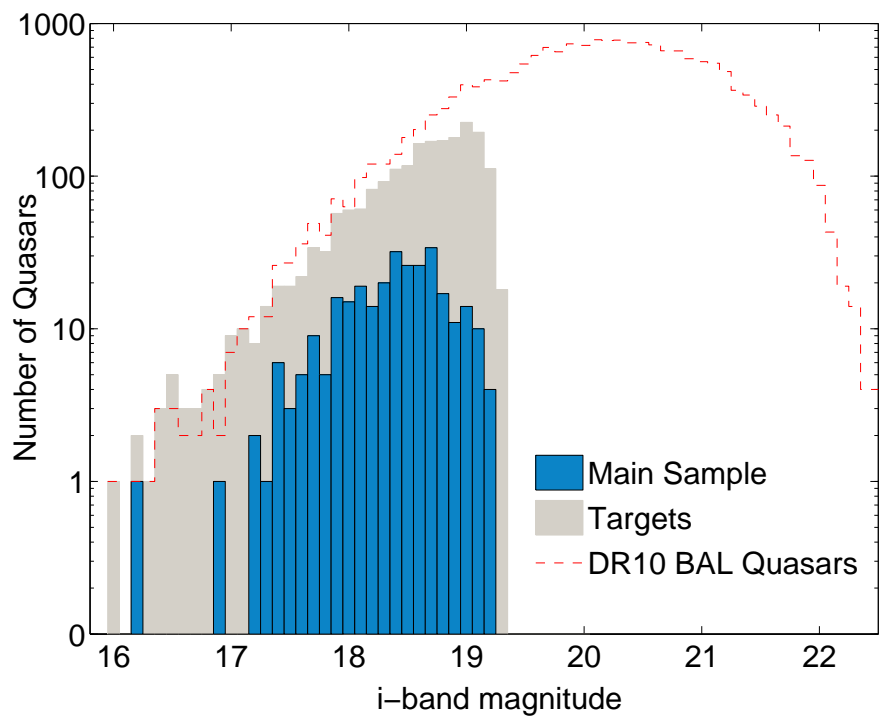

Fig. 2.- Comparison of the $i$-band apparent magnitude distributions of the main sample of this paper (blue), the 2005 targets of the BOSS ancillary project on BAL quasars (gray), and the BAL quasars identified in the SDSS DR10 quasar catalog (dashed red line). Note that we are targeting the brightest BAL quasars in the SDSS sky area in order to obtain spectra of the highest possible quality.

2650-2910 $\AA, 3950-4050 \AA$. The RLF windows were selected to be relatively free from emission and absorption lines considering the composite quasar spectra of Vanden Berk et al. (2001). However, some weak emission and/or absorption lines can be present in these spectral regions. Thus, to exclude the data points that deviate from the fit by more than $3 \sigma$, we apply an iterative sigma-clipping algorithm using a non-linear least squares fit. We fit the RLF windows of each spectrum with an intrinsically reddened power-law continuum model where we use Small-Magellanic-Cloud type reddening. We calculate the continuum uncertainties using $\Delta \chi^{2}$ confidence-region estimation for $68.3 \%$ confidence bounds. Throughout this work, we propagate the continuum uncertainties into the uncertainties on restframe EW measurements. As in previous studies (e.g., Lundgren et al. 2007; Filiz Ak et al. 2012), we do not model the emission lines since investigation of emission- line characteristics is beyond the scope of this study (also see Section 2.2).

\subsection{Identification and Measurements of BAL Troughs}

As is common practice (e.g., Trump et al. 2006; Gibson et al. 2008, 2009; Allen et al. 2011; Filiz Ak et al. 2012), we smoothed each spectrum using a SavitzkyGolay algorithm to perform local linear regression for three consecutive data points (see Section 3.2 of Filiz Ak et al. 2012). We utilize normalized and smoothed spectra only for identification of BAL troughs; unsmoothed spectra are used for further calculations. Only BAL troughs in the velocity range -3000 to $-30000 \mathrm{~km} \mathrm{~s}^{-1}$ are considered (see Section 2.2); the small fraction $(\approx 2.5 \%)$ of extremely high-velocity C IV BAL troughs exceeding the $-30000 \mathrm{~km} \mathrm{~s}^{-1}$ limit were removed by considering Si IV BAL troughs at corresponding velocities. 
The canonical definition of BAL troughs (see Equation (2) was developed for the purpose of finding BAL troughs in a single-epoch spectrum. However, our primary purpose here is investigating BAL variability in multi-epoch spectra. Therefore, we adopt a modified BAL-trough definition more appropriate for our purpose that is strongly motivated by the canonical definition and reduces to it for single-epoch data. Our adopted BAL-trough definition utilizes the information from all available spectral observations of a quasar. As is well known, absorption troughs are sometimes isolated and sometimes appear in complexes in which single troughs may split or adjacent troughs may merge over time. To address these complications, we treat each BAL complex as a single BAL trough. We identify BAL complexes using the following algorithm (see Figure 3):

1. We first identify BAL and mini-BAL troughs (hereafter just "troughs") under the canonical definition in each single-epoch spectrum of a quasar 18 We set the maximum velocity of a trough to be $v_{\text {max,t }}$ and the minimum velocity to be $v_{\min , \mathrm{t}}$.

2 . We select $v_{\text {min,t }}$ of the highest velocity trough in the first-epoch spectrum and compare the corresponding velocities in all the available spectra. If the $v_{\text {min,t }}$ velocity intersects any trough region in the other available epochs, we re-assign $v_{\min , \mathrm{t}}$ to be the lowest velocity of this intersecting trough and repeat the comparison to the other available spectra. If the $v_{\text {min,t }}$ velocity does not intersect any trough region in the other available spectra, we set $v_{\min , \mathrm{t}}$ to be the minimum red-edge velocity of the BAL complex, $v_{\min }$.

3. To define the maximum velocity of the complex, we take the $v_{\text {max,t }}$ of the lowest-velocity trough associated with the complex and compare with the other available epochs. If the $v_{\max , \mathrm{t}}$ velocity in the other spectra intersects a trough, we set $v_{\max , \mathrm{t}}$ to be the highest velocity of this intersecting trough. If the $v_{\text {max,t }}$ velocity does not intersect any trough region in the other available spectra, we set $v_{\text {max,t }}$ to be the maximum blue-edge velocity of the BAL complex, $v_{\max }$.

In this algorithm each trough can be associated with only one trough complex. Each trough complex includes at least one trough which is wider than $2000 \mathrm{~km} \mathrm{~s}^{-1}$ lying between -3000 and $-30000 \mathrm{~km} \mathrm{~s}^{-1}$.

After implementing the above algorithm, we define each BAL complex lying between $v_{\max }$ and $v_{\min }$ as a distinct BAL trough. Here, $v_{\max }$ is the maximum velocity taken to be the velocity at the blue edge for any trough associated with the complex across all available spectra of each quasar. Similarly, $v_{\min }$ is defined using the red-edge velocities. After an automated identification of BAL troughs using our algorithm above, we visually inspect all the available spectra of each main-sample quasar. The inspection shows that our approach for BAL-trough identification is appropriately implemented for both CIV and Si IV BAL troughs. We found that, in

18 Mini-BALs are defined using Equation 2 but for a trough width of 500-2000 $\mathrm{km} \mathrm{s}^{-1}$ (cf. Hamann \& Sabra 2004). the majority of cases, the complex would be identified as a single BAL trough under the canonical definition in at least one of our epochs. Moreover, our adopted BALtrough definition produces the same results as the canonical definition for non-merging and non-splitting BAL troughs that lie between constant $v_{\max }$ and $v_{\min }$ in all the available spectral observations. Using our adopted BAL-trough definition we identified a total of 428 distinct C IV and 235 distinct Si IV BAL troughs in the 699 main-sample spectra.

Figure 3 illustrates our adopted BAL-trough definition and the canonical one using all the available spectra of the quasar SDSS J090944.05+363406.7. If we apply the canonical BAL-trough definition in this example, the two adjacent BAL troughs seen in the last epoch would be identified as two distinct BALs. However, the same structure appears as one distinct BAL trough in the second-epoch spectrum and in the first-epoch spectrum appears as two mini-BALs along with one BAL trough. As is clearly seen in this example, our adopted BALtrough definition produces more physically meaningful results than the canonical definition for the purpose of studying variability in multi-epoch spectra. Moreover, we select the $v_{\max }$ and $v_{\min }$ velocities using information from all the available spectra instead of only a singleepoch observation. If we were to take the $v_{\max }$ and $v_{\min }$ velocities as the blue-edge and red-edge velocities of the complex where all absorption has merged to one BAL trough (i.e., the second-epoch spectrum in this example), we would lose the pertinent information from the part of the BAL troughs which extend beyond these velocities in the other-epoch spectra (i.e., the first-epoch spectrum in this example).

In this study, we investigate BAL-trough variability on a large range of rest-frame timescales. The spectral observations from SDSS-I/II and BOSS provide coverage of long timescales, typically longer than $1 \mathrm{yr}$. To sample shorter timescales, we use the additional observations from SDSS and/or BOSS that are available for more than $20 \%$ of our main-sample quasars. To avoid the repeat examination of BAL troughs and the associated multi-counting biases, we utilize only the twoepoch spectra for each quasar that give the minimum sampled rest-frame timescale, $\Delta t_{\min } 19$ Although we use only such two-epoch spectra for our calculations below, we still consider all available observations in our BALtrough identification algorithm as we have found this provides the most physically meaningful results. By selecting $\Delta t_{\mathrm{min}}$, we sample rest-frame timescales from a few hours to a few years. Figure 4 shows the distribution of minimum sampled rest-frame timescales, $\Delta t_{\mathrm{min}}$, for distinct BAL troughs identified in our main-sample spectra. The $\Delta t_{\min }$ values range between $5.9 \mathrm{hr}$ and $3.7 \mathrm{yr}$ with a median of $2.1 \mathrm{yr}$. Given that C IV BAL troughs are not always accompanied by Si IV BAL troughs, we ran a two-

\footnotetext{
19 For example, if a quasar has observations that sample restframe timescales of $0.01,0.02,0.03,2.00,2.01$, and $2.03 \mathrm{yr}$, we select only the two-epoch spectra that sample the $0.01 \mathrm{yr}$ timescale. Note that the $2.00,2.01$, and $2.03 \mathrm{yr}$ timescales are nearly the same (agreeing to within 1.5\%) and provide little independent information. Thus, using all three of these timescales would result in this object being inordinately weighted in statistical characterizations of BAL variability on $\approx 2 \mathrm{yr}$ timescales (i.e., causing multi-counting bias).
} 


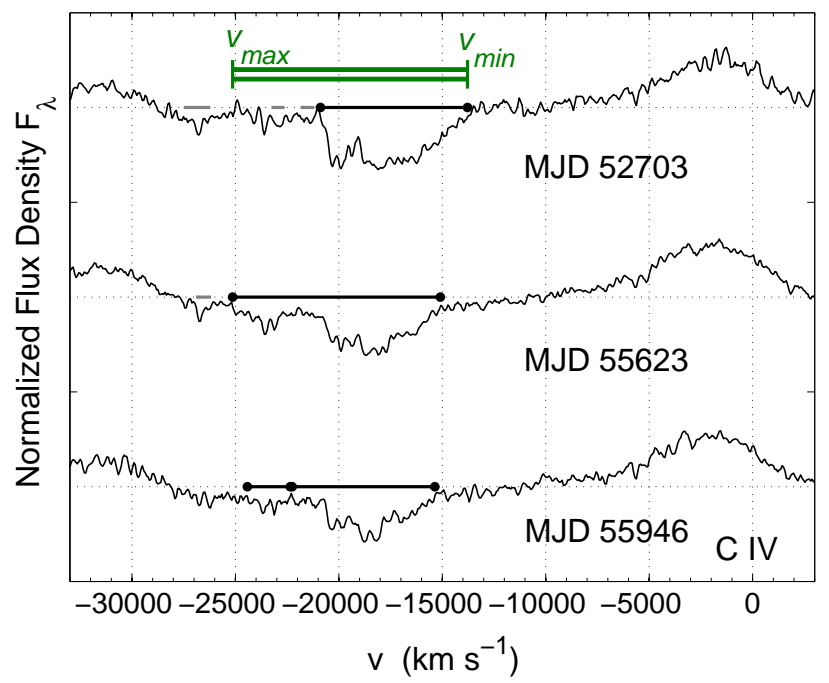

FIG. 3. - Example of our adopted BAL-trough definition illustrated using the three available spectra of the quasar SDSS J090944.05+363406.7. The three normalized spectra for this quasar are arbitrarily offset in flux for clarity of presentation. Horizontal dotted lines show the continuum levels for each spectrum, and the tick marks on the $y$-axis show the zero level for each spectrum. Horizontal black bars show absorption lines with $\Delta v \geq 2000 \mathrm{~km} \mathrm{~s}^{-1}$, corresponding to the traditional BAL definition, and horizontal gray bars show absorption lines with $\Delta v=500-2000 \mathrm{~km} \mathrm{~s}^{-1}$. The variable absorption complex is complicated; in some spectra only part of it is classified as a BAL under the canonical definition. We use all three available spectra to define the minimum and maximum velocities of the trough. The horizontal double green bar shows the resulting BAL trough lying between $v_{\min }$ and $v_{\max }$ (see Section 3.2).

sample Kolmogorov-Smirnov (KS) test to compare the sampled timescale distributions of C IV and Si IV BAL troughs and found no significant difference.

In some sections of this study, we focus on BALtrough variability characteristics solely on multi-year timescales. Therefore, we define another timescale of $\Delta t_{\min , 1}$ to sample minimum rest-frame timescales of more than 1 yr. By this definition, we select the same non-repeating sample of distinct BAL quasars as with the $\Delta t_{\min }$ selection. $\Delta t_{\min , 1}$ ranges between $1-3.7 \mathrm{yr}$ with a median of $2.3 \mathrm{yr}$. Compared to previous studies (e.g., Lundgren et al. 2007; Gibson et al. 2008, 2009; Capellupo et al. 2011, 2012), and especially those focusing on multi-year timescales, we have a significantly larger (by about an order-of-magnitude) BAL-trough sample.

We measure the rest-frame EW of each BAL trough in each epoch and calculate the uncertainties on EWs using Equations 1 and 2 of Kaspi et al. (2002), where uncertainties are derived by propagating the continuumestimation errors (see Section 3.1) and the observational errors of each contributing pixel. In addition, we measure the weighted centroid velocity, $v_{\text {cent }}$, for each BAL trough; i.e., the mean of the velocities where each data point is weighted with its distance from the normalized continuum level. We also calculate an average BALtrough depth, $d_{\mathrm{BAL}}$, which is the mean distance from the normalized continuum level for each data point of a BAL trough.

\subsection{Identification and Measurements of Variable BAL} Troughs
As one approach to identify variable BAL troughs in our main sample, we select BAL troughs showing significant $\mathrm{EW}$ variations. To calculate $\mathrm{EW}$ variations, $\Delta \mathrm{EW}$, and uncertainties on this quantity, $\sigma_{\Delta \mathrm{EW}}$, we use the following equations:

$$
\Delta \mathrm{EW}=\mathrm{EW}_{2}-\mathrm{EW}_{1}, \quad \sigma_{\Delta \mathrm{EW}}=\sqrt{\sigma_{\mathrm{EW}_{2}}^{2}+\sigma_{\mathrm{EW}_{1}}^{2}(3)}
$$

where $\mathrm{EW}_{1}$ and $\mathrm{EW}_{2}$ are the EWs measured from twoepoch spectra that are observed at times $t_{1}$ and $t_{2}$. In our sample, the mean $\sigma_{\Delta \mathrm{EW}}$ is $0.5 \AA$ for $\mathrm{C}$ IV and $0.4 \AA$ for Si IV BAL troughs. Similarly, we calculate fractional $\mathrm{EW}$ variations, $\frac{\Delta \mathrm{EW}}{\langle\mathrm{EW}}$, and corresponding uncertainties, $\sigma_{\frac{\Delta \mathrm{EW}}{\langle\mathrm{EW}\rangle}}$, with the following equations:

$$
\begin{array}{r}
\frac{\Delta \mathrm{EW}}{\langle\mathrm{EW}\rangle}=\frac{\left(\mathrm{EW}_{2}-\mathrm{EW}_{1}\right)}{\left(\mathrm{EW}_{2}+\mathrm{EW}_{1}\right) \times 0.5}, \\
\sigma_{\frac{\Delta \mathrm{EW}}{\langle\mathrm{EW}\rangle}}=\frac{4 \times\left(\mathrm{EW}_{2} \sigma_{\mathrm{EW}_{1}}+\mathrm{EW}_{1} \sigma_{\mathrm{EW}_{2}}\right)}{\left(\mathrm{EW}_{2}+\mathrm{EW}_{1}\right)^{2}}
\end{array}
$$

We identify 248 variable CIV BAL troughs and 119 variable Si IV BAL troughs showing $\mathrm{EW}$ variations at a significance level of more than $3 \sigma$ on timescales of more than 1 yr $\left(\Delta t_{\min , 1}\right)$. Similarly, we identify 223 variable C IV BAL troughs and 99 variable Si IV BAL troughs by comparing the two-epoch spectra that sample the $\Delta t_{\min }$ timescales in the rest frame.

Considering that variations tend to occur in portions of BAL troughs (e.g., Gibson et al. 2008; Capellupo et al. 2011), as an alternative approach, we define a variable BAL trough to have at least one variable region. For the purpose of determining regions in each BAL trough where a variation has occurred, we compare two-epoch spectra of each quasar. Since a proper comparison requires consideration of the signal-to-noise ratio of each spectrum, we define a measurement of the deviation between two observations for each pixel in units of $\sigma$ using the following equation:

$$
N_{\sigma}(\lambda)=\frac{f_{2}-f_{1}}{\sqrt{\sigma_{2}^{2}+\sigma_{1}^{2}}}
$$

where $f_{1}$ and $f_{2}$ are the normalized flux densities and $\sigma_{1}$ and $\sigma_{2}$ are the normalized flux-density standard deviations at wavelength $\lambda$. Both $\sigma_{1}$ and $\sigma_{2}$ include observational errors and uncertainties on the estimated continuum model. Similarly to Gibson et al. (2008), we identify variable regions of BAL troughs to be where an absorption feature is detected with $N_{\sigma} \geq 1$ or $N_{\sigma} \leq-1$ for at least five consecutive data points. This requirement allows detection of variable regions wider than $\approx 275 \mathrm{~km} \mathrm{~s}^{-1}$. Selection using a smaller number of consecutive data points as the requirement may cause nonphysical observational errors to be indistinguishable from the variable regions of BAL troughs. On the other hand, requiring a larger number of consecutive data points will cause non-detection of narrow variable regions. By requiring the number of data points to be $\geq 5$, we require the significance of variations to be $>99.9 \%$.

We have identified 903 variable regions for C IV BAL troughs and 294 variable regions for Si IV BAL troughs for variations on timescales of more than $1 \mathrm{yr}\left(\Delta t_{\min , 1}\right)$. We also identified 757 variable regions for CIV BAL 


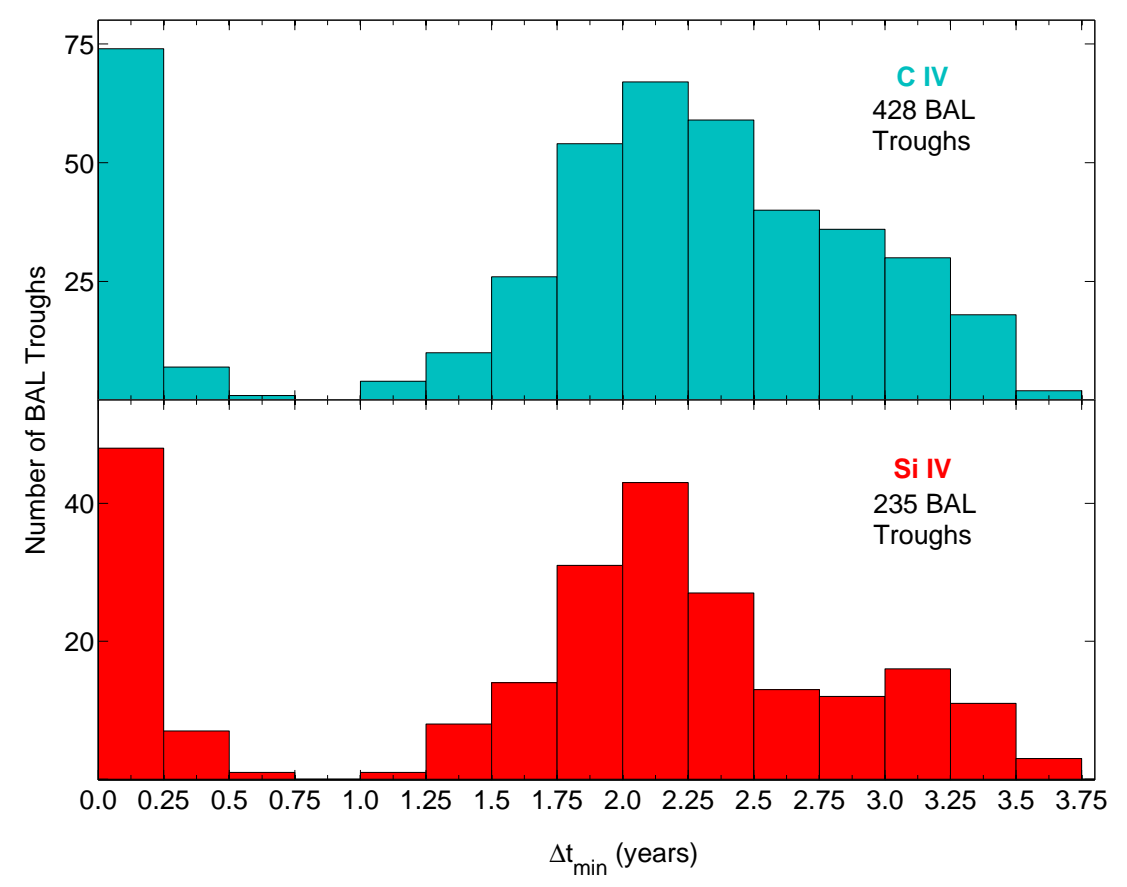

FIG. 4.- Distributions of the minimum sampled rest-frame timescale, $\Delta t_{\min }$, for 428 distinct C IV (upper panel) and 235 distinct Si IV (lower panel) BAL troughs present in the spectra of the main-sample quasars. We have significant trough statistics on timescales as long as $3-3.5 \mathrm{yr}$.

troughs and 232 variable regions for Si IV BAL troughs by comparing the two-epoch spectra that sample the $\Delta t_{\mathrm{min}}$ timescales in the rest frame. The number of BAL troughs having at least one variable region is 294 for C IV and 119 for Si IV on timescales of more than $1 \mathrm{yr}$.

Comparing the two approaches to variable BAL-trough identification, we found that $26 \mathrm{C} \mathrm{IV} \mathrm{BAL} \mathrm{troughs} \mathrm{show-}$ ing EW variations at more than $3 \sigma$ significance do not have a variable region satisfying our requirements, although several narrow variable regions in these BAL troughs collectively produce $\mathrm{EW}$ variations at more than $3 \sigma$ significance. We also found that $72 \mathrm{C} \mathrm{IV} \mathrm{BAL} \mathrm{troughs}$ having one variable region do not show EW variations at more than $3 \sigma$ significance. In these 72 cases, a narrow variable region in a strong BAL trough cannot produce an $\mathrm{EW}$ variation at more than $3 \sigma$ significance due to statistical dilution by the rest of the trough. Similarly, we found that $29 \mathrm{Si}$ IV BAL troughs showing $\mathrm{EW}$ variations at more than $3 \sigma$ significance do not have a variable region satisfying our requirements, and $29 \mathrm{Si}$ IV BAL troughs having one variable region do not show EW variation at more than $3 \sigma$ significance.

We will refer to both of these approaches to variable BAL trough identification in the following sections. We present our measurements for C IV and Si IV BAL troughs in Tables 2 and 3 and for $\mathrm{C}$ IV and Si IV variable regions in Tables 4 and 5, respectively.

\section{RESULTS ON BAL VARIABILITY}

In this section, we present the results of our BAL variability investigations utilizing the multi-epoch observations of 428 distinct C IV and 235 distinct Si IV BAL troughs in the 699 main-sample spectra of 291 quasars. We examine the fraction of variable BAL troughs and BAL quasars (Section 4.1), the velocity widths of the variable regions of BAL troughs (Section 4.2), EW vari- ations as a function of timescales (Section 4.3), the distribution of $\mathrm{EW}$ variations (Section 4.4), EW variations as a function of BAL profile properties (Section 4.5), relative EW variations between $\mathrm{C}$ IV and Si IV BAL troughs (Section 4.6), correlated EW variations in BAL quasars with multiple troughs (Section 4.7), and EW variations as a function of quasar properties (Section 4.8).

\subsection{Fraction of BAL Troughs and BAL Quasars Showing Variability}

We calculate the fraction of BAL troughs showing variability and the fraction of quasars showing BAL-trough variability in our main sample considering the two different variability identification approaches explained in Section 3.3. First, requiring a variable BAL trough to show an $\mathrm{EW}$ variation at more than $3 \sigma$ significance, we find that the fraction of variable BAL troughs is $57.9_{-3.7}^{+3.9} \%$ for $\mathrm{C}$ IV and $50.6_{-4.6}^{+5.1} \%$ for Si IV on timescales of $1-3.7 \mathrm{yr}$. Figure 5 presents the cumulative fraction of variable BAL troughs; the $y$-axis shows the cumulative fraction of BAL troughs with EW variations of more than a given threshold $|\Delta \mathrm{EW}|$. Although the cumulative fraction of variable BAL troughs decreases for large $|\Delta \mathrm{EW}|$, it remains significant even for threshold $|\Delta \mathrm{EW}|$ values as large as $5 \AA$.

We also compare the fraction of variable C IV and Si IV BAL troughs from the same absorbing material. Given that the Si IV region between -13000 and $-30000 \mathrm{~km} \mathrm{~s}^{-1}$ can have contamination by emission and/or absorption lines such as C II (1335 ̊), O I (1306 $\AA$ ), and Ly $\alpha+\mathrm{N}$ v, we select a sample of $136 \mathrm{C}$ IV BAL troughs lying between -3000 and $-13000 \mathrm{~km} \mathrm{~s}^{-1}$ that are accompanied by Si IV BAL troughs at corresponding velocities. In this sample, we found that the fraction of BAL troughs showing EW variations at more than $3 \sigma$ significance is $50.0_{-6.0}^{+6.8} \%$ for $\mathrm{C}$ IV and $53.7_{-5.4}^{+6.3 \%}$ for Si IV on timescales of $1-3.7 \mathrm{yr}$. 

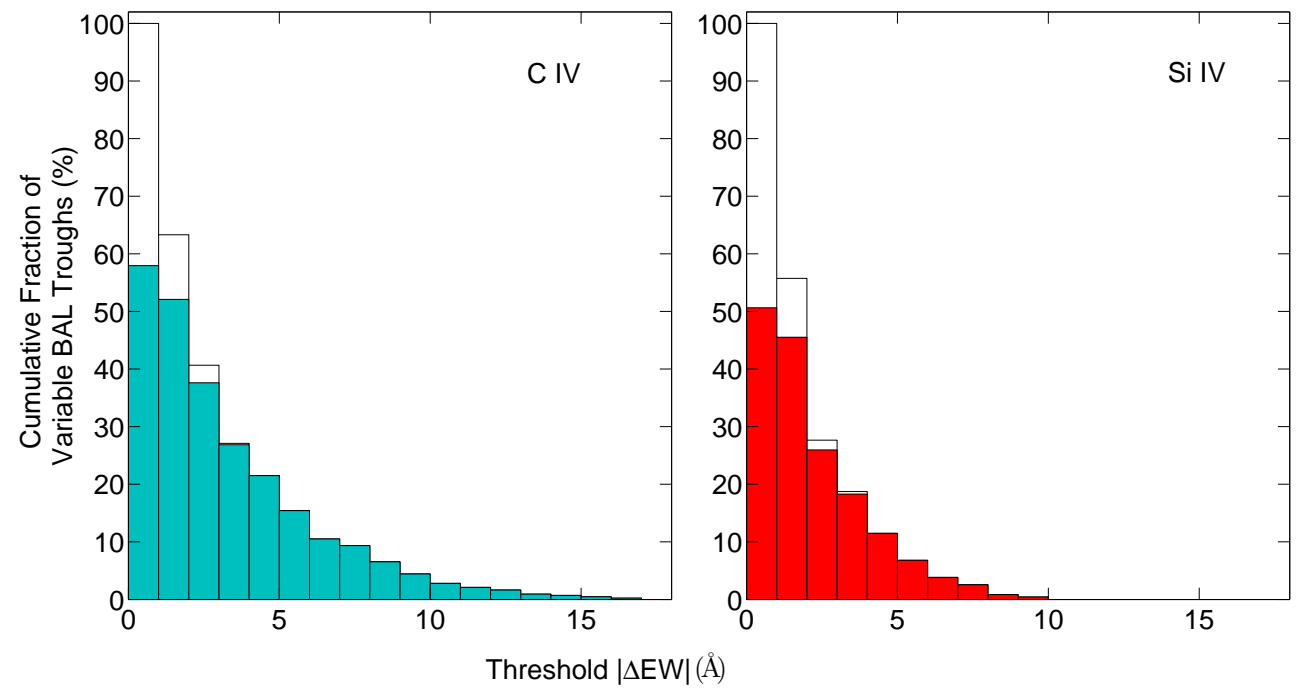

FIG. 5.- Cumulative fraction of BAL troughs with a given threshold $|\Delta \mathrm{EW}|$ for CIV and Si IV troughs. The open histograms show the cumulative fraction of BAL troughs with EW variations of more than a given threshold. The shaded parts of the histograms indicate the fraction of BAL troughs showing EW variations of more than $3 \sigma$ significance on timescales of 1-3.7 $\mathrm{yr}$.

These fractions indicate that C IV and Si IV BAL troughs at corresponding velocities are about equally likely to vary.

In addition to considering the fraction of variable BAL troughs, we also calculate the fraction of quasars showing BAL-trough variability. Requiring a variable BAL trough to show an $\mathrm{EW}$ variation at more than $3 \sigma$ significance, we found the fraction of quasars showing $\mathrm{C}$ IV BAL variations to be $62.2_{-4.6}^{+4.9} \%(181 / 291)$. Considering a total of 181 quasars showing Si IV BAL absorption, we found the fraction of quasars showing Si IV BAL variations to be $59.1_{-5.7}^{+6.3} \%$ (107/181). Using multi-epoch observations of 24 quasars, Capellupo et al. (2011) found that the fraction of quasars showing C IV absorption variations is $39 \%$ on timescales of $0.35-0.75$ yr and $65 \%$ on timescales of 3.8-7.7 yr. Capellupo et al. (2012) reported that 11 out of $19(58 \%)$ quasars exhibited Si IV absorption variations on timescales of 3.8-7.7 yr. Considering that our data sample timescales of 1-3.7 yr with an average of $2.3 \mathrm{yr}$, both of our results broadly show consistency with the results of the Capellupo et al. (2011) study.

Alternatively, we consider variable BAL troughs to be those with at least one variable region detected in the trough (see Section 3.3). We find that the fraction of BAL troughs showing variability is $68.6_{-4.0}^{+4.3} \%$ for C IV and $50.6_{-4.6}^{+5.1 \%}$ for Si IV on timescales of $1-3.7 \mathrm{yr}$. This approach is more sensitive to local variations in $\mathrm{BAL}$ troughs; a narrow variable region in a wide BAL trough may not produce an EW variation at more than $3 \sigma$ significance.

We further investigate the number of variable regions as a function of velocity, $v$, that is measured relative to the quasar redshift. Figure 6 presents the number of variable regions found at a particular velocity for C IV and Si IV BAL troughs for variations on timescales of more than 1 yr $\left(\Delta t_{\min , 1}\right)$. C IV variable regions are found across a wide range of velocities, and the number of variable regions appears to peak in the range between -9000 and $-21000 \mathrm{~km} \mathrm{~s}^{-1}$ in concert with the number of BAL troughs. Figure 6 also displays the per- centage of BAL regions showing variability for C IV and $\mathrm{Si}$ IV that is calculated from the ratio of the number of variable regions to the number of BAL troughs found at a particular velocity. We found that the percentage of C IV BAL regions showing variability is roughly constant at around $30-40 \%$ at velocities of $-3000 \mathrm{~km} \mathrm{~s}^{-1}$ to $-25000 \mathrm{~km} \mathrm{~s}^{-1}$ and rises in the few highest-velocity bins; we have verified that this trend is statistically significant. Capellupo et al. (2011) also investigated the fraction of variations as a function of outflow velocity on short (0.35-0.75 yr) and long (3.8-7.7 yr) timescales. Consistent with our results, they found that CIV BAL troughs tend to be more variable at higher velocities.

The Si IV region can have contamination by emission and/or absorption lines, and the superposition of these emission lines and Si IV BALs may prevent BAL troughs from continuously lying at least $10 \%$ under the continuum level and thus may lead to non-detection. A visual inspection showed that in some cases BAL troughs appear to be broken into two or more narrow sections by such emission lines, causing the apparent absorption features to fail to satisfy the criteria to be identified as a BAL trough. Consequently, these effects also cause a decrease in the number of variable Si IV BAL troughs. Due to these effects, the number of variable Si IV BAL troughs and therefore variable Si IV regions decreases between -13000 and $-30000 \mathrm{~km} \mathrm{~s}^{-1}$; the histogram for Si IV should not be interpreted physically in this velocity range. Thus, we show the percentage distribution of Si IV BAL troughs only for a region between -3000 and $-13000 \mathrm{~km} \mathrm{~s}^{-1}$.

\subsection{Velocity Widths of Variable Regions of BALs}

Gibson et al. (2008) showed that variations tend to occur only in portions of BAL troughs. To investigate the distribution of velocity widths of variable BAL regions, we calculate $\Delta v_{\mathrm{VR}}$ values for 903 variable regions in C IV BAL troughs and 294 variable regions in Si IV BAL troughs for variations on timescales of more than $1 \mathrm{yr}$, where $\Delta v_{\mathrm{VR}}$ is the velocity width of a variable region. Figure 7 shows the $\Delta v_{\mathrm{VR}}$ distributions of variable regions 


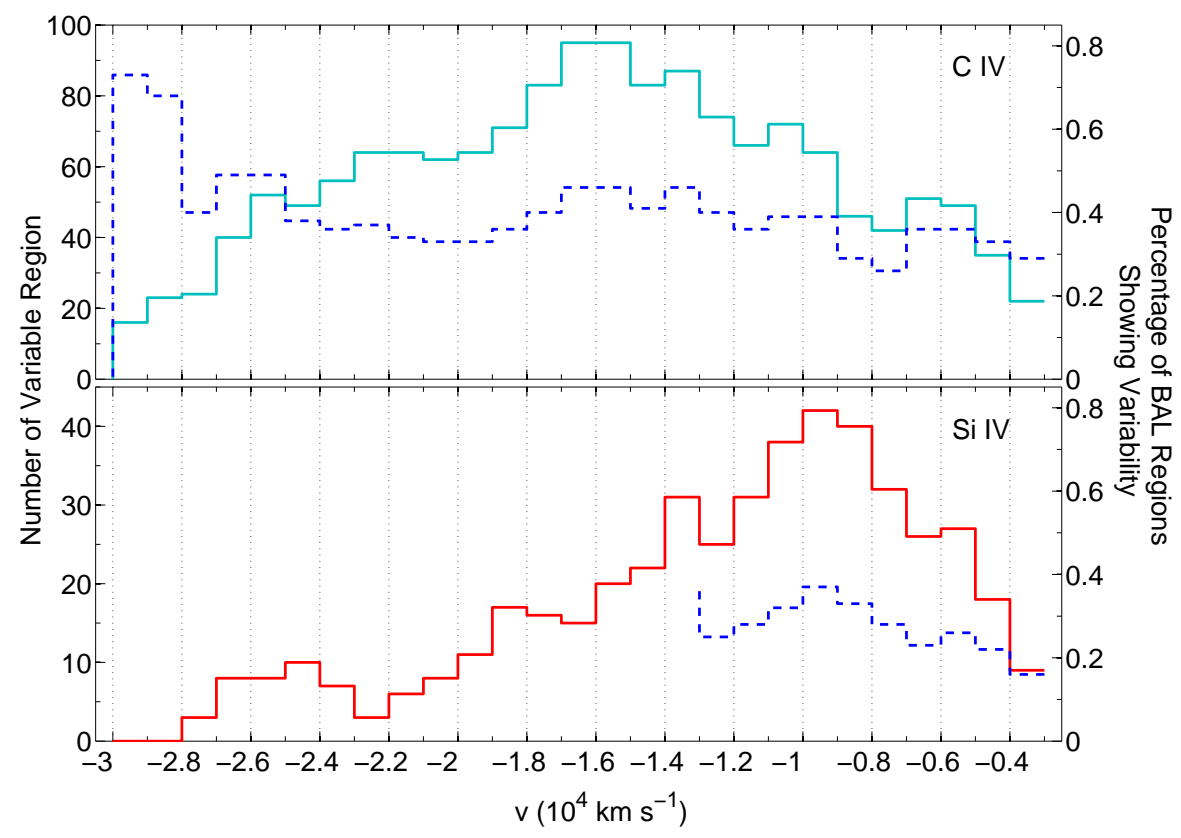

FIG. 6. - Number of times a variable region is found at a particular velocity in C IV (upper panel) and Si IV (lower panel) BAL troughs for variations on timescales of more than $1 \mathrm{yr}$. In the upper panel, the dashed dark-blue line along with the right $y$-axis shows the percentage of BAL regions showing variability in CIV. C IV variable regions are found across a wide range of velocities. The Si IV region can have contamination by emission lines such as C II (1335 $\AA$ ), O I (1306 $⿱$ ) ), and Ly $\alpha+\mathrm{N} v$, causing the apparent decrease in the number of variable Si IV regions between -13000 and $-30000 \mathrm{~km} \mathrm{~s}^{-1}$; the histogram for Si IV should not be interpreted physically in this velocity range. Therefore, we do not show the percentage of variable regions for Si IV between -13000 and $-30000 \mathrm{~km} \mathrm{~s}^{-1}$.

detected in C IV and Si IV BAL troughs. Consistent with Gibson et al. (2008), we find that the number of variable regions, in both $\mathrm{C}$ IV and Si IV, rises toward small velocity widths down to our velocity width measurement limit of $\approx 275 \mathrm{~km} \mathrm{~s}^{-1}$ (see Section 3.3). We found that the mean of the $\Delta v_{\mathrm{VR}}$ measurements for C IV and Si IV BAL troughs are $713.6 \mathrm{~km} \mathrm{~s}^{-1}$ and $592.8 \mathrm{~km} \mathrm{~s}^{-1}$, respectively.

To examine the fraction of a BAL trough that is variable, we define $f_{\Delta v}$ as the sum of the velocity widths of all varying regions in a BAL trough divided by the BAL trough velocity width, $\Delta v$. Figure 8 shows the $f_{\Delta v}$ distribution for C IV BAL troughs on timescales of $1-3.7 \mathrm{yr}$ and the $f_{\Delta v}$ distribution as a function of $\Delta v$ for C IV. We find that the mean of $f_{\Delta v}$ is 0.20 for CIV and 0.13 for Si IV, indicating that Si IV variable regions on average tend to be narrower than C IV variable regions. Figure 8 also suggests that narrow C IV BAL troughs tend to have a larger fraction of variable regions compared to wide C IV BAL troughs.

We also investigate the position of each variable region in a given BAL trough to assess if the incidence of variability depends upon the relative velocity within the trough. We calculate the normalized relative velocity in the trough, $v_{\text {nrt }}$. Here $v_{\text {nrt }}$ is $\frac{v_{\text {cent }}-v_{\text {mid }}}{\left|v_{\text {cent }}-v_{\max }\right|}$ for the blue part of the trough and $\frac{v_{\text {cent }}-v_{\text {mid }}}{v_{\text {cent }}-v_{\min }}$ for the red part of the trough, where $v_{\text {mid }}$ is the mid velocity of a variable region. Figure 9 shows the number of variable regions found at a given $v_{\text {nrt }}$. A non-parametric triples test (Randles et al. 1980) shows that the distribution of $v_{\text {nrt }}$ shows no significant evidence of asymmetry $(P=0.55)$, and the distribution is relatively constant across almost the entire width of a trough. Consistent with our results, Capellupo et al. (2011) found no evidence for a higher incidence of variability with positive or negative velocity offset, but we establish this result with substantially better statistics.

\subsection{EW Variations as a Function of Timescale}

Previous BAL-variability studies (e.g., Gibson et al. 2008, 2010; Capellupo et al. 2011) have found that C IV BAL-trough variability is larger for longer timescales. This is expected since, e.g., quasar variability in general is larger on longer timescales (e.g., Vanden Berk et al. 2004). In order to investigate EW variations as a function of timescale with a larger sample over a wide range of rest-frame timescales, we utilize the $\Delta \mathrm{EW}$ and $\Delta \mathrm{EW} /\langle\mathrm{EW}\rangle$ values of each distinct BAL trough from the two-epoch spectra for timescales of $\Delta t_{\min }$ (see Equations 3 and (4). In Figure 10, we show EW variations for CIV and Si IV BAL troughs as functions of $\Delta t_{\min }$. For comparison, we also include the data from Barlow (1993) that correspond to a timescale range mainly between $0.2-1 \mathrm{yr}$ and from Gibson et al. (2008) that extend the timescales up to $6.1 \mathrm{yr}$. To display the spread of $\Delta \mathrm{EW}$, we calculate the standard deviation of our data using a sliding window containing 20 time-ordered data points; we statistically remove the mean EW error in each window from the standard deviation (via standard error propagation). The curves of standard deviation indicate an increase of $\mathrm{EW}$ variations with increasing rest-frame timescale both for CIV and SiIV BAL troughs. This trend is consistent with that of previous BAL-variability studies. The majority of the Barlow (1993) data lie between the standard-deviation curves that are calculated from our data. Although the data from Gibson et al. (2008) sample longer timescales than our data, the trend of the standard-deviation curves shows general agreement regarding the increase of EW variations with in- 


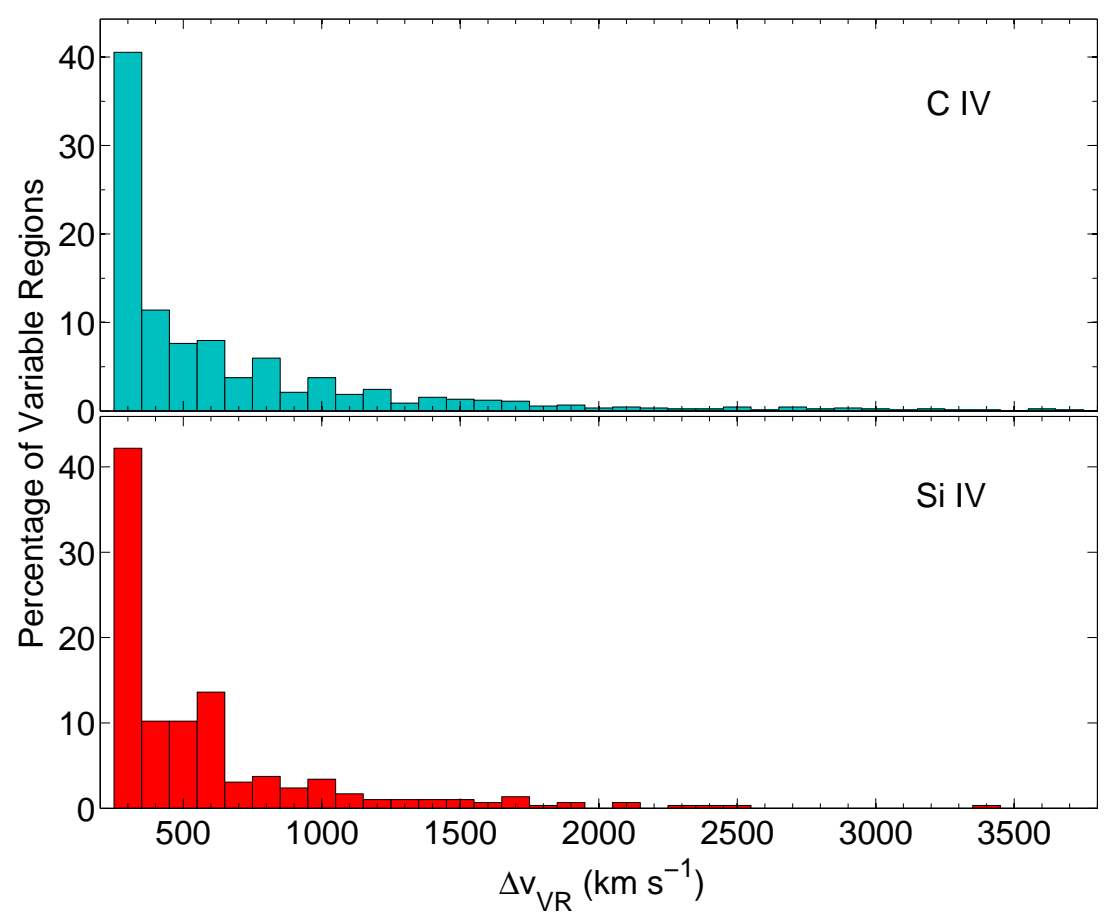

FIG. 7.- Percentage of variable regions with a given velocity width in C IV (upper panel) and Si IV (lower panel) BAL troughs for variations on timescales of more than $1 \mathrm{yr}$. We found seven variable regions for C IV and one variable region for Si IV with velocity widths of $4500-7500 \mathrm{~km} \mathrm{~s}^{-1}$ that we do not show in this figure. For both C IV and Si IV, the number of variable regions rises rapidly toward small velocity widths down to our velocity width measurement limit.
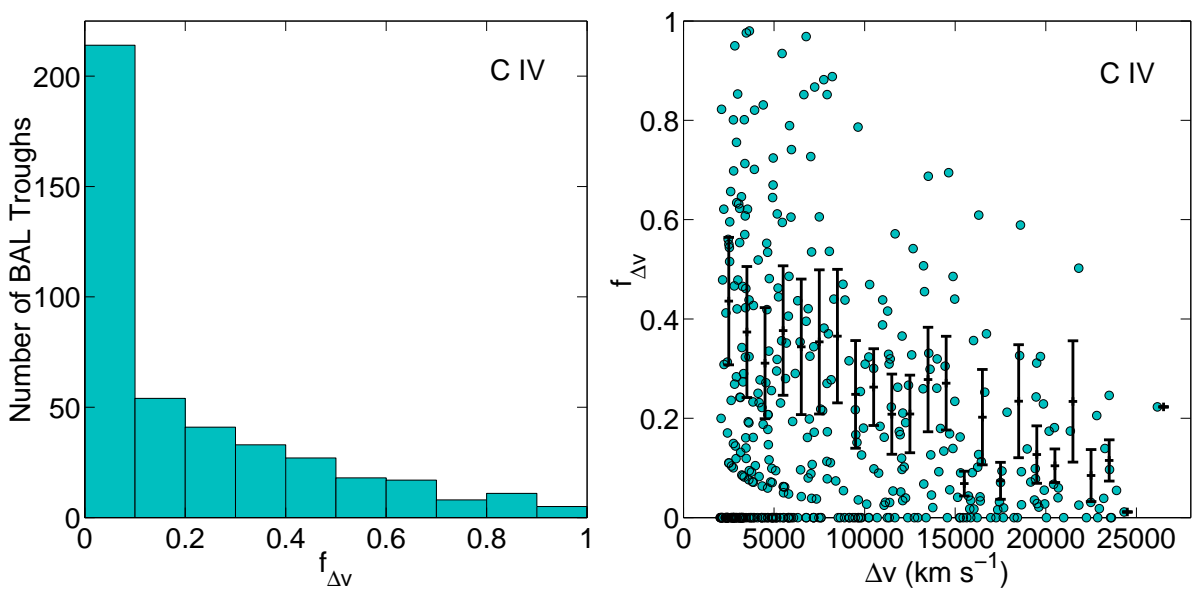

FIG. 8. - Distribution of $f_{\Delta v}$ (left panel), and $f_{\Delta v}$ distribution as a function of BAL trough width $\Delta v$ (right panel). Vertical black bars in the right panel show the standard deviation around the mean for non-zero $f_{\Delta v}$ values in given $100 \mathrm{~km} \mathrm{~s}^{-1}$ wide $\Delta v$ bins. Both of the panels are for C IV BAL troughs on timescales of $1-3.7 \mathrm{yr}$.

creasing timescale.

Figure [1] shows fractional EW variations for C IV and Si IV BAL troughs against $\Delta t_{\min }$. The curves of standard deviation are illustrated both for C IV and Si IV BAL troughs. The spread of the curves indicates an increase of fractional EW variations with increasing rest-frame timescales. As for Figure 10, we include the data from Barlow (1993) and Gibson et al. (2008) for comparison purposes.

Figures 10 and 11demonstrate that our data points are consistent with zero variation for timescales $\Delta t_{\text {min }}<0.01$ yr (i.e., 3.6 days) in the rest frame given the measurement errors. This result is expected given the fact that troughs are unlikely to show significant variations on timescales of hours or days. Therefore, any observed variations on these timescales provides an empirical estimate of the total systematic and measurement errors of our data and data-processing methods. For a more quantitative assessment, we calculated the median deviation in units of $\sigma$ for $\Delta t_{\min }<0.01 \mathrm{yr}$ and found that it is $\approx 1$ for both $\mathrm{C}$ IV and Si IV.

To quantify the relationship between EW variations and timescale, we calculate the mean of $|\Delta \mathrm{EW}|$ and $\Delta t_{\text {min }}$ for bins containing 15 time-ordered data points. From a robust linear-regression model using the bisquare 


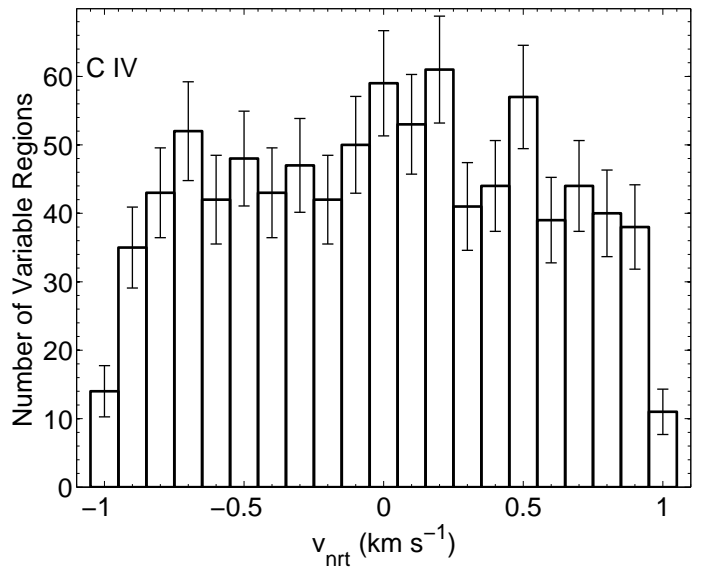

FIG. 9.- The number of variable regions found at a given $v_{\text {nrt }}$. The distribution is relatively constant across the entire trough width.

weight function (Press et al. 1992), we found a fit of

$$
\begin{array}{r}
\log \left|\Delta \mathrm{EW}_{\mathrm{C} \text { IV }}\right|=(0.258 \pm 0.031) \times \log \Delta t_{\min } \\
+(0.289 \pm 0.027)
\end{array}
$$

where the units of $|\Delta \mathrm{EW}|$ and $\Delta t_{\text {min }}$ are $\AA$ and yr, respectively. Similarly, for fractional EW variations we found a fit of

$$
\begin{aligned}
& \log \left|\frac{\Delta \mathrm{EW}}{\langle\mathrm{EW}\rangle}\right|_{\mathrm{C} \text { IV }}=(0.283 \pm 0.042) \times \log \Delta t_{\text {min }} \\
& +(-0.737 \pm 0.037) \text {. }
\end{aligned}
$$

In Figure 12, we display the fraction of C IV BAL troughs showing variability at more than $3 \sigma$ significance as a function of timescale. We calculate the mean timescale and the fraction of variable BAL troughs for 20 time-ordered data points for variations on timescales of less than $1 \mathrm{yr}$, and for two equal-size bins for variations of more than $1 \mathrm{yr}$. Consistent with Gibson et al. (2010) and Capellupo et al. (2013), our results indicate the incidence of variability increases with time.

We also investigate the rate-of-change of EW variations on short and long timescales by calculating $|\Delta \mathrm{EW}| / \Delta t$ for $\mathrm{C}$ IV and Si IV BAL troughs on timescales of $\Delta t_{\min }=0.01-1 \mathrm{yr}$ and $\Delta t_{\min }>1 \mathrm{yr}$. Figure 13 displays the distribution of $|\Delta \mathrm{EW}| / \Delta t$ for $\mathrm{C}$ IV and Si IV BAL troughs on both short and long timescales. It also presents the distribution of $|\Delta \mathrm{EW}| / \Delta t$ for BAL troughs with $\geq 3 \sigma$ significance variations of $\mathrm{EW}$ in each panel. The distributions for variations with $\geq 3 \sigma$ significance show that the average rate-of-change of $\mathrm{EW}$ variations is larger on short timescales. Gibson et al. (2010) found a similar result from the comparison of variations of BAL troughs for eight individual sources on short and long timescales (see their Figure 9). Such behavior would be expected, for example, if BAL EWs execute a simple random walk with a step timescale of $\lesssim 1$ yr (see Section 5.4). On timescales longer than the step timescale then the observed $|\Delta \mathrm{EW}| / \Delta t \propto 1 / \sqrt{n}$, where $n$ is the number of steps during the observed period.

\subsection{Distribution of EW Variations}

In this section, we investigate several characteristics of BAL EW variability distributions. We first examine the symmetry of distributions of BAL EW variations to assess and constrain any differences between the formation and decay timescales of BAL troughs. Figure 14 presents the distributions of $428 \mathrm{C}$ IV and $235 \mathrm{Si}$ IV BALtrough $\mathrm{EW}$ variations, $\triangle \mathrm{EW}$, and fractional $\mathrm{EW}$ variations, $\Delta \mathrm{EW} /\langle\mathrm{EW}\rangle$, for variations on timescales of more than 1 yr. To maintain an equilibrium of BAL troughs in quasar spectra, we expect the weakening and strengthening of BAL troughs in a large sample to be balanced. To assess whether BAL-trough variations are balanced, we examine the mean values of the $\Delta \mathrm{EW}$ and $\Delta \mathrm{EW} /\langle\mathrm{EW}\rangle$ distributions. We find that the mean of the $\Delta \mathrm{EW}$ distribution for CIV BAL troughs is $-0.082 \pm 0.184 \AA$ and for SiIV BAL troughs is $0.101 \pm 0.163 \AA$. The error on the mean is calculated following $\sigma / \sqrt{N}$ where $N$ is the number of BAL troughs. Similarly, we find the mean of the $\Delta \mathrm{EW} /\langle\mathrm{EW}\rangle$ distribution for C IV BAL troughs is $-0.032 \pm 0.018$ and for Si IV BAL troughs is $-0.003 \pm 0.029$. These results indicate that the mean values of the $\Delta \mathrm{EW}$ and $\Delta \mathrm{EW} /\langle\mathrm{EW}\rangle$ distributions for $\mathrm{C} I V$ and Si IV BAL troughs are broadly consistent with zero.

We next investigated if the $\Delta \mathrm{EW}$ and $\Delta \mathrm{EW} /\langle\mathrm{EW}\rangle$ distributions are symmetric. An asymmetric distribution could be seen, for instance, if the strengthening and weakening of BAL troughs occur at different rates. For example, if a typical BAL trough forms rapidly and decays slowly, the distribution of BAL variations would be skewed to negative $\triangle \mathrm{EW}$ (i.e., the number of weakening BAL troughs would be larger at any given time). Gibson et al. (2010) showed that the distribution of $\Delta \mathrm{EW}$ for $23 \mathrm{C}$ IV BAL troughs is reasonably symmetric.

As can be seen in Figure 14, the $\Delta \mathrm{EW}$ and $\Delta \mathrm{EW} /\langle\mathrm{EW}\rangle$ distributions do not show clear evidence of asymmetry. For a more quantitative examination, we use a non-parametric triples test. The results of this test on the $\Delta \mathrm{EW}$ distributions for $\mathrm{C}$ IV and Si IV BAL troughs show no significant evidence of asymmetry $(P=0.45$ and $P=0.40$, respectively). Similarly, we found no significant evidence of asymmetry for the $\Delta \mathrm{EW} /\langle\mathrm{EW}\rangle$ distributions for C IV and Si IV BAL troughs $(P=0.25$ and $P=0.04$, respectively).

As another approach to assess asymmetry, we compare the distributions of strengthening and weakening BAL troughs by running a two-sample KS test for the distributions of BAL troughs with increasing and decreasing EWs. The test results show that the positive and negative parts of the $\Delta \mathrm{EW}$ distributions for $\mathrm{C} I \mathrm{~V}$ and Si IV BAL troughs do not show significant inconsistency ( $P=0.58$ and $P=0.83$, respectively). Similarly, we find that the positive and negative parts of the $\Delta \mathrm{EW} /\langle\mathrm{EW}\rangle$ distributions for $\mathrm{C}$ IV and Si IV BAL troughs do not show significant inconsistency $(P=0.12$ and $P=0.60$, respectively).

Given that the test results show no significant evidence of asymmetry, we calculate the skewness of the $\Delta \mathrm{EW}$ and $\Delta \mathrm{EW} /\langle\mathrm{EW}\rangle$ distributions to establish a basic limit upon the amount of asymmetry allowed by our data. We found that the skewness of the $\Delta \mathrm{EW}$ distribution is $-0.17 \pm 0.12$ for $\mathrm{C}$ IV BAL troughs and $-0.17 \pm 0.16$ for Si IV BAL troughs. For the calculation of the error on skewness, we follow the standard approximate $\sqrt{6 / N}$ formula (Press et al. 1992). The skewness of the $\Delta \mathrm{EW} /\langle\mathrm{EW}\rangle$ distribution is $-0.33 \pm 0.12$ for $\mathrm{C}$ IV BAL 


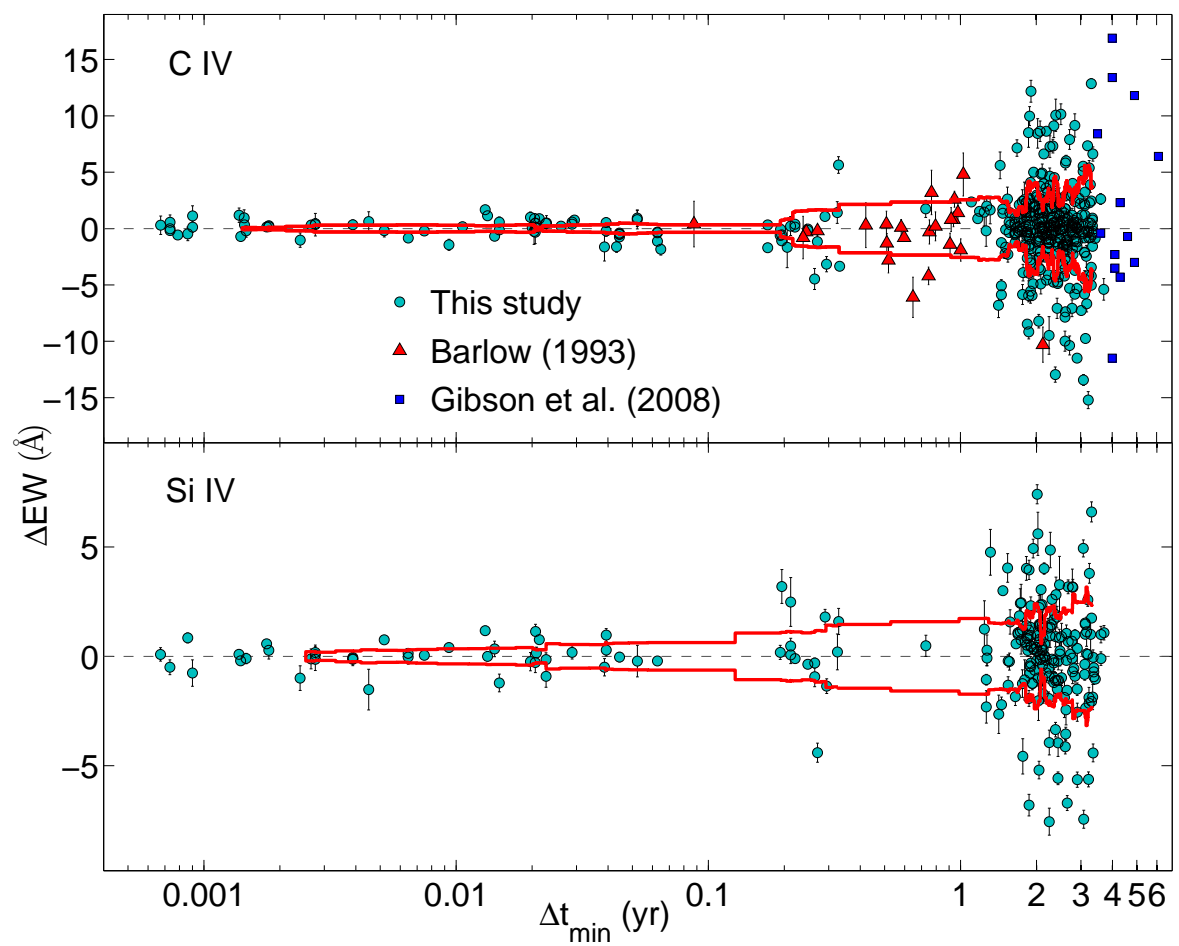

FIG. 10.- EW variation, $\Delta \mathrm{EW}$, vs. the minimum sampled rest-frame timescale, $\Delta t_{\mathrm{min}}$, for $\mathrm{C}$ IV (upper panel) and Si IV (lower panel) BAL troughs. The data are from this study (light blue circles), Barlow (1993; red triangles), and Gibson et al. (2008; dark blue squares). The red solid curves indicate the standard deviation derived from the data in this study, calculated using a sliding window containing 20 time-ordered data points. The standard deviation of $\Delta \mathrm{EW}$ increases with increasing rest-frame timescale.

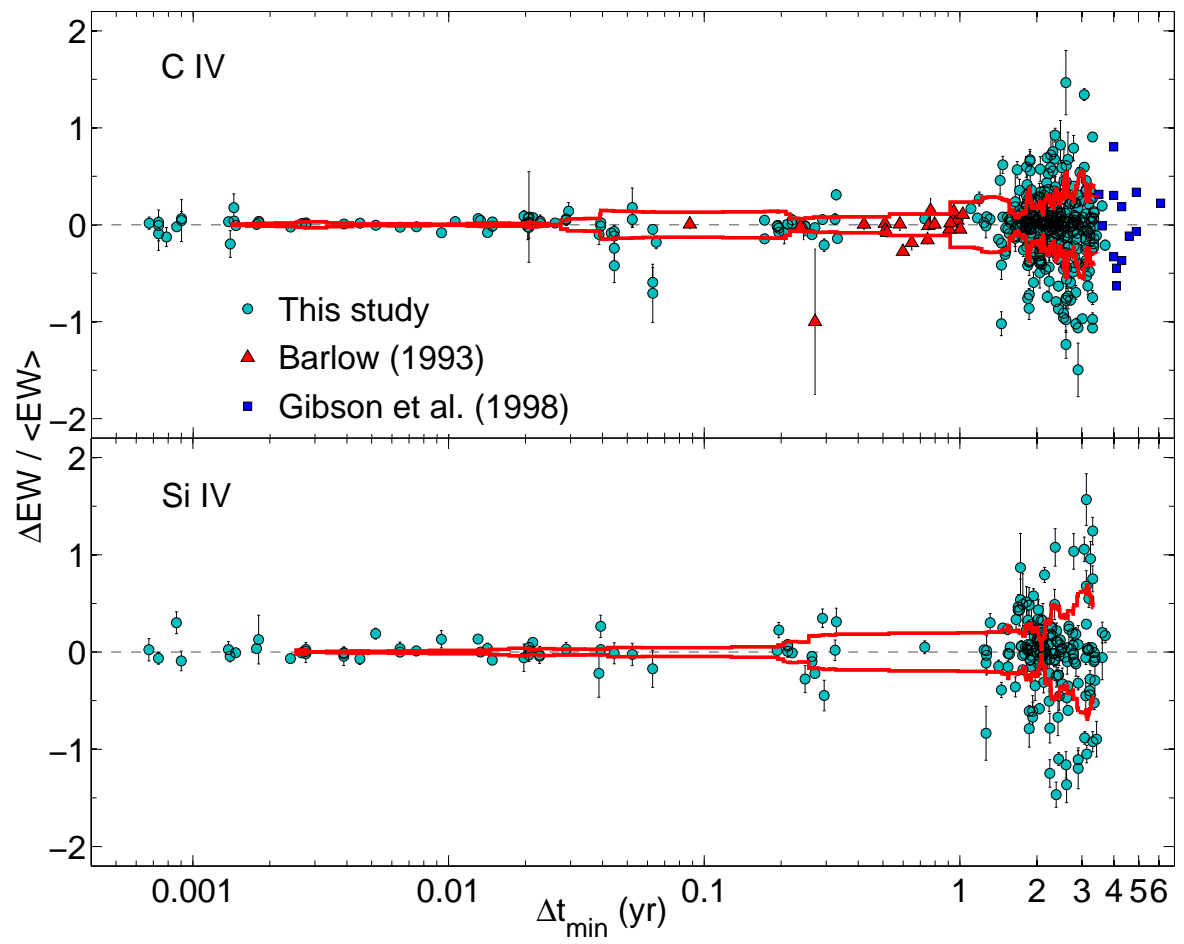

FIG. 11. - Same as Figure 5 but for fractional EW variation, $\Delta \mathrm{EW} /\langle\mathrm{EW}\rangle$.

troughs and $-0.32 \pm 0.16$ for Si IV BAL troughs.

We next investigated whether $\Delta \mathrm{EW}$ and $\Delta \mathrm{EW} /\langle\mathrm{EW}\rangle$ are Gaussian distributed; following the central-limit theorem, many random variability processes will generate
Gaussian distributions. Although their sample of BAL troughs was not large enough to draw firm conclusions about the characteristics of BAL variation distributions, Gibson et al. (2010) suggested that $\Delta \mathrm{EW}$ may not be 


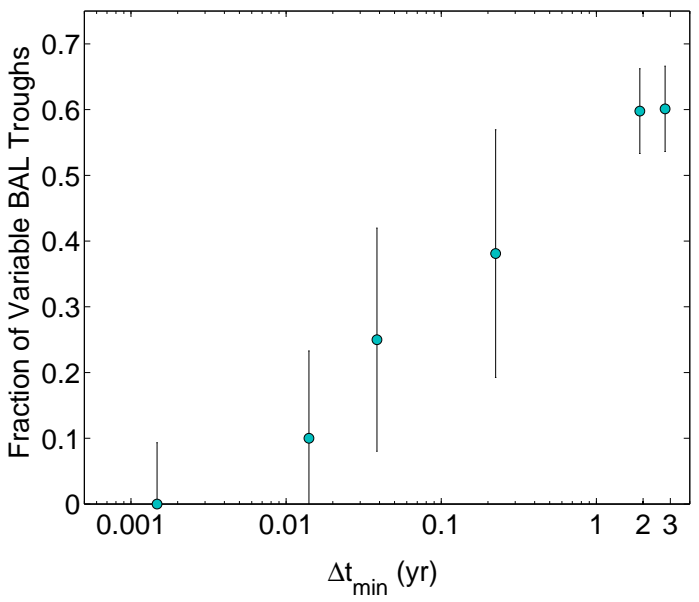

FIG. 12.- The fraction of C IV BAL troughs showing variability at more than $3 \sigma$ significance as a function of timescale. We calculate the counting errors following Gehrels (1986).

normally distributed over time spans of months-to-years. We analyze the shape of the $\Delta \mathrm{EW}$ and $\Delta \mathrm{EW} /\langle\mathrm{EW}\rangle$ distributions for $\mathrm{C}$ IV and Si IV BAL troughs considering variations longer than $1 \mathrm{yr}$ using the Lilliefors normality test (Lilliefors 1967). The test shows that both the $\Delta \mathrm{EW}$ and $\Delta \mathrm{EW} /\langle\mathrm{EW}\rangle$ distributions for $\mathrm{C}$ IV and Si IV BAL troughs are non-Gaussian (at a significance level of $>99.9 \%$ ).

Figure 14 shows the best Gaussian model distributions for comparison purposes in each panel. Since the mean values of the $\Delta \mathrm{EW}$ distributions are consistent with zero, we calculate the best Gaussian models using $\mu=0$ and the measured standard deviation of each distribution. For both CIV and Si IV BAL troughs, the distributions of $\Delta \mathrm{EW}$ have a stronger central peak, and both of the distributions are weaker than the Gaussian distributions for $|\Delta \mathrm{EW}| \approx 2-7 \AA$. Similarly, the $\Delta \mathrm{EW} /\langle\mathrm{EW}\rangle$ distributions for $\mathrm{C}$ IV and Si IV BAL troughs are stronger for $|\Delta \mathrm{EW} /\langle\mathrm{EW}\rangle|<0.2$ and weaker for a range of $|\Delta \mathrm{EW} /\langle\mathrm{EW}\rangle| \approx 0.2-0.6$ compared to the Gaussian distributions.

A non-Gaussian distribution of $\mathrm{EW}$ variations on timescales of more than $1 \mathrm{yr}$ may appear as a result of the superposition of several Gaussian distributions, each of which characterizes the $\mathrm{EW}$ variations in a small time interval (see Figure 10). In order to test this hyphothesis, we ran a Lilliefors normality test for the $\Delta \mathrm{EW}$ and $\Delta \mathrm{EW} /\langle\mathrm{EW}\rangle$ distributions in a small time interval. We selected the time interval of $2.0-2.5 \mathrm{yr}$, since a large number of BAL troughs are sampled in this range (see Figures (10) and 11); $156 \mathrm{C}$ IV and $90 \mathrm{Si}$ IV BAL troughs are sampled. The Lilliefors test showed that the $\Delta \mathrm{EW}$ and $\Delta \mathrm{EW} /\langle\mathrm{EW}\rangle$ distributions for C IV and Si IV BAL-trough variations on $\Delta t_{\min , 1}=2.0-2.5 \mathrm{yr}$ are also non-Gaussian distributions (significance level of $>95 \%$ ).

We have also investigated the $\Delta \mathrm{EW}$ and $\Delta \mathrm{EW} /\langle\mathrm{EW}\rangle$ distributions for $\mathrm{CIV}$ and SiIV BAL troughs on timescales of $\Delta t_{\mathrm{min}}<1 \mathrm{yr}$. Performing the triples and Lilliefors tests, we found that the $\Delta \mathrm{EW}$ and $\Delta \mathrm{EW} /\langle\mathrm{EW}\rangle$ distributions are also symmetric and non-Gaussian for both CIV and Si IV BAL troughs (significance level of $>99.9 \%$ ).

\subsection{EW Variations as a Function of BAL-Profile Properties}

In this section, we investigate $\mathrm{EW}$ variations of $\mathrm{BAL}$ troughs as a function of BAL-profile properties, such as average EW, velocity width, depth, and centroid velocity. Although Gibson et al. (2008) found no significant correlation between the magnitude of $\mathrm{EW}$ variations and the average EW of C IV BAL troughs, we search for correlations for both C IV and Si IV BAL troughs with a much larger sample for variations in three different timescale ranges. Figure 15 shows $\Delta \mathrm{EW}$ as a function of $\langle\mathrm{EW}\rangle$ for short $(<1 \mathrm{yr})$, moderate $(1-2.5 \mathrm{yr})$, and long $(>2.5 \mathrm{yr})$ timescales. Here the average $\mathrm{EW},\langle\mathrm{EW}\rangle$, indicates the average of the measured EWs of each BAL trough in two-epoch spectra. The number of data points used is given in the lower right of each panel. We illustrate with dotted curves where the EW variation is equal to the average EW. We search for correlations using the Spearman rank-correlation test between $|\Delta \mathrm{EW}|$ and $\langle\mathrm{EW}\rangle[\mathrm{EO}$ The test results show that the correlations are highly significant (>99\%) for CIV BAL-trough EW variations on short, moderate and long timescales. The $|\Delta \mathrm{EW}|$ for Si IV BAL troughs also likely correlates with $\langle\mathrm{EW}\rangle$ on moderate timescales with a significance level of $>95 \%$. We do not detect significant correlations for variations of Si IV BAL troughs on short and long timescales, although these cases have the poorest trough statistics. These results suggest that weak BAL troughs tend to have small EW variations, an expected finding given that BAL troughs cannot weaken by more than their initial EWs measured in the first-epoch spectra. Remarkably, it is apparent in Figure 15 that weak BAL troughs also do not rise above the equality line. In addition, Figure 15 indicates that the $\mathrm{EW}$ variations of weak BAL troughs can be close to their average EWs.

Studies by Lundgren et al. (2007) and Gibson et al. (2008) suggested a significant correlation between the fractional variation of $\mathrm{EW}, \Delta \mathrm{EW} /\langle\mathrm{EW}\rangle$, and $\langle\mathrm{EW}\rangle$. To investigate this correlation in a large sample, in Figure 16 we show $\Delta \mathrm{EW} /\langle\mathrm{EW}\rangle$ as a function of $\langle\mathrm{EW}\rangle$ for $\mathrm{C}$ IV and Si IV BAL troughs for three timescales. In agreement with these previous studies, we find that the correlations between $|\Delta \mathrm{EW} /\langle\mathrm{EW}\rangle|$ and $\langle\mathrm{EW}\rangle$ for C IV BAL troughs are highly significant $(>99.9 \%)$ for all three timescale ranges. The test results demonstrate that the correlations between $|\Delta \mathrm{EW} /\langle\mathrm{EW}\rangle|$ and $\langle\mathrm{EW}\rangle$ for Si IV BAL troughs are also highly significant $(>99.9 \%)$ for variations on moderate and long timescales. The significance level of the correlation is $98.4 \%$ for variations of Si IV troughs on short timescales. Figure [16] shows that the fractional variations of small-EW BAL troughs are larger compared to large-EW BAL troughs. Consistent with our results, the studies by Lundgren et al. (2007) and Gibson et al. (2008) found the $|\Delta \mathrm{EW} /\langle\mathrm{EW}\rangle|$ vs. $\langle\mathrm{EW}\rangle$ correlation. However, they likely did not see significant correlation for $|\Delta \mathrm{EW}|$ vs. $\langle\mathrm{EW}\rangle$ due to the small sample size.

Given that the width and depth of a BAL trough de-

20 Although we assess correlations between $|\Delta \mathrm{EW}|$ and BALtrough profile properties, we prefer to use $\Delta \mathrm{EW}$ in our figures instead. This allows us to examine possible dependencies of strengthening vs. weakening (for example, see the middle panel of Figure 17) and associated discussion. 


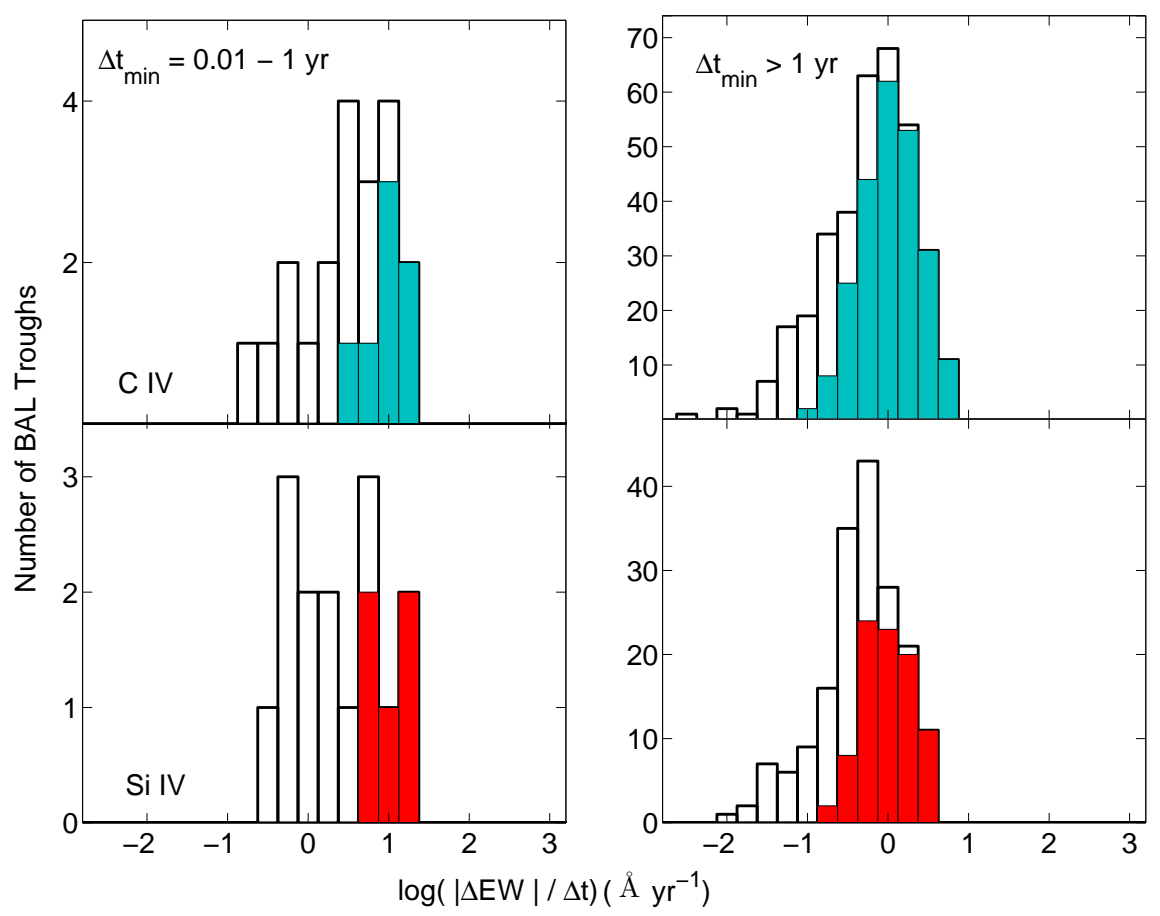

FIG. 13.- Distributions of rate-of-change of $|\mathrm{EW}|,|\Delta \mathrm{EW}| / \Delta t$, for C IV (upper panels) and Si IV (lower panels) BAL troughs both on short (left panels) and long (right panels) timescales. The shaded parts of the histograms show the BAL troughs with $\geq 3 \sigma$ significance variations of EW.

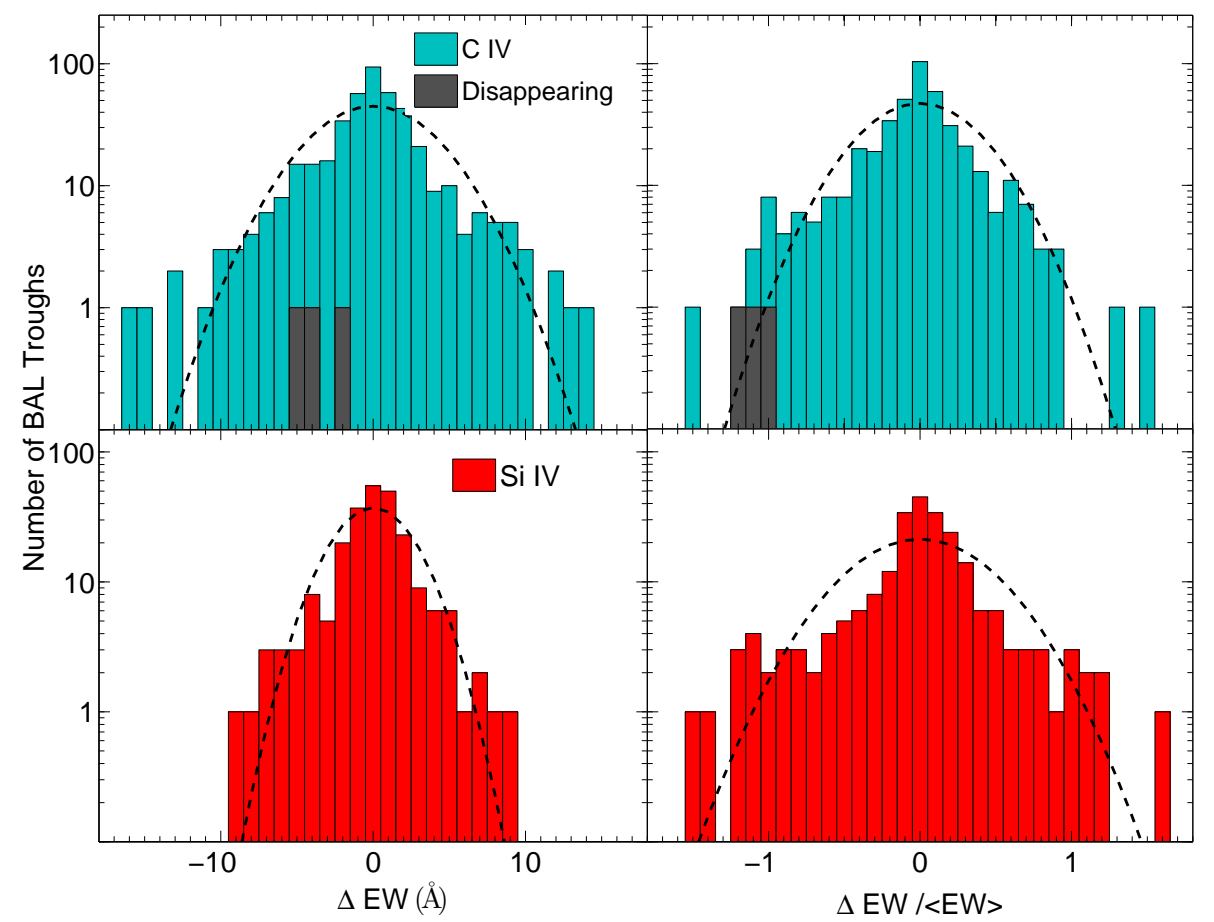

FIG. 14.- Distributions of BAL-trough EW variations, $\Delta \mathrm{EW}$, and fractional EW variations, $\Delta \mathrm{EW} /\langle\mathrm{EW}\rangle$, for $\mathrm{C}$ IV (upper panels) and Si IV (lower panels) for variations on timescales of more than $1 \mathrm{yr}$. Black dashed lines show the best Gaussian fits to the data. Our main sample includes three of the disappearing C IV BAL troughs described in Filiz Ak et al. (2012); these are plotted with a dark gray histogram in the upper panels (see Section 5.3 for further discussion). Our main sample also includes one additional new case of C IV BAL disappearance that satisfies the disappearance criteria used in Filiz Ak et al. (2012); this case is plotted in the upper panel at $\Delta \mathrm{EW} /\langle\mathrm{EW}\rangle=-1.5$ and is found in the quasar SDSS J095901.24+550408.2. The cases of BAL disappearance appear to be an extreme of the overall distribution of BAL variability, rather than a distinct population of variability events. None of the disappearing C IV BAL troughs has a Si IV BAL trough at corresponding velocities at our observed epochs.

termine its EW, we assess the contributions of these two components to the significant correlation found between
$|\Delta \mathrm{EW}|$ and $\langle\mathrm{EW}\rangle$ for $\mathrm{C}$ IV BAL troughs. We search for correlations between BAL-trough width, $\Delta v$, and BAL- 


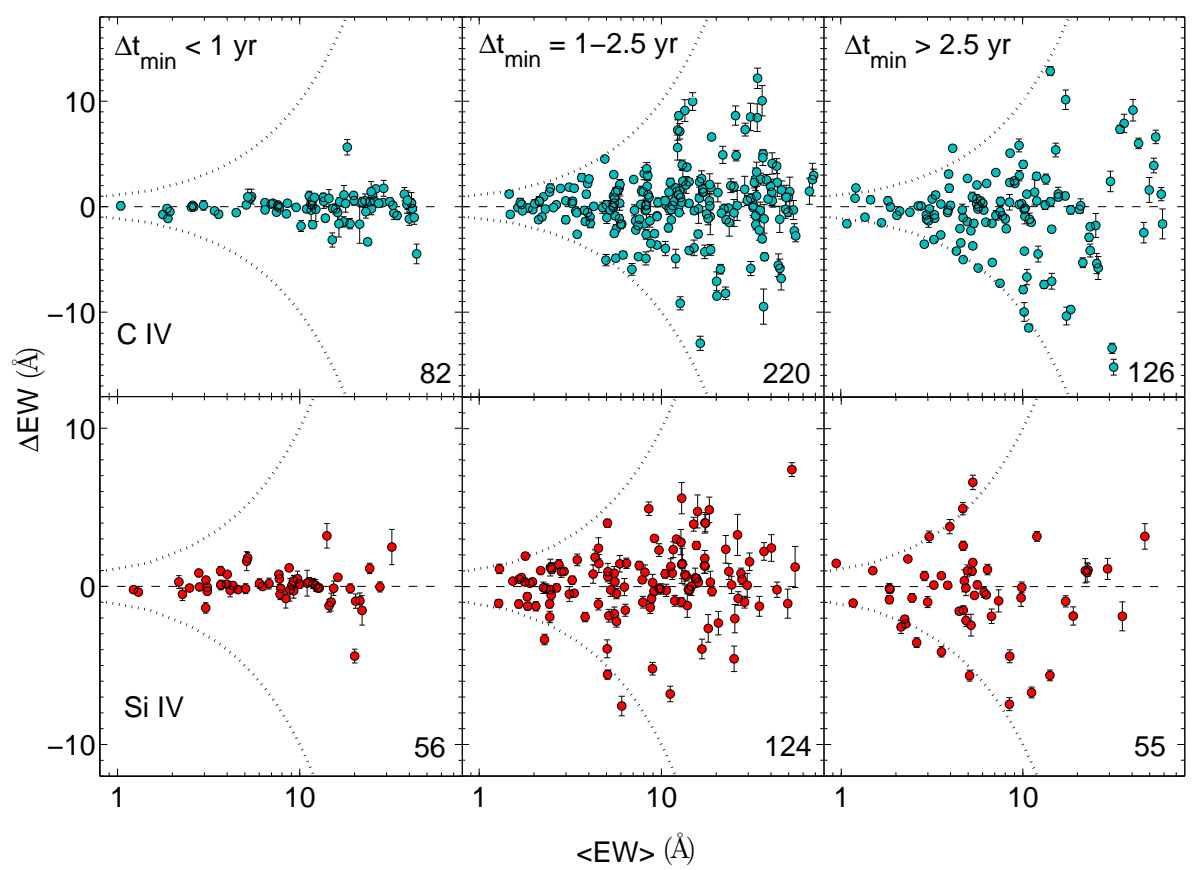

Fig. 15. - EW variation, $\Delta \mathrm{EW}$, vs. average EW over the two relevant epochs, $\langle\mathrm{EW}\rangle$, for $\mathrm{C}$ IV (blue) and Si IV (red) BAL troughs for three different timescales as labeled. The $x$-axis is logarithmic. The black dotted curves denote where $|\Delta \mathrm{EW}|$ is equal to $\langle\mathrm{EW}\rangle$. The number of data points are given in the lower right of each panel.

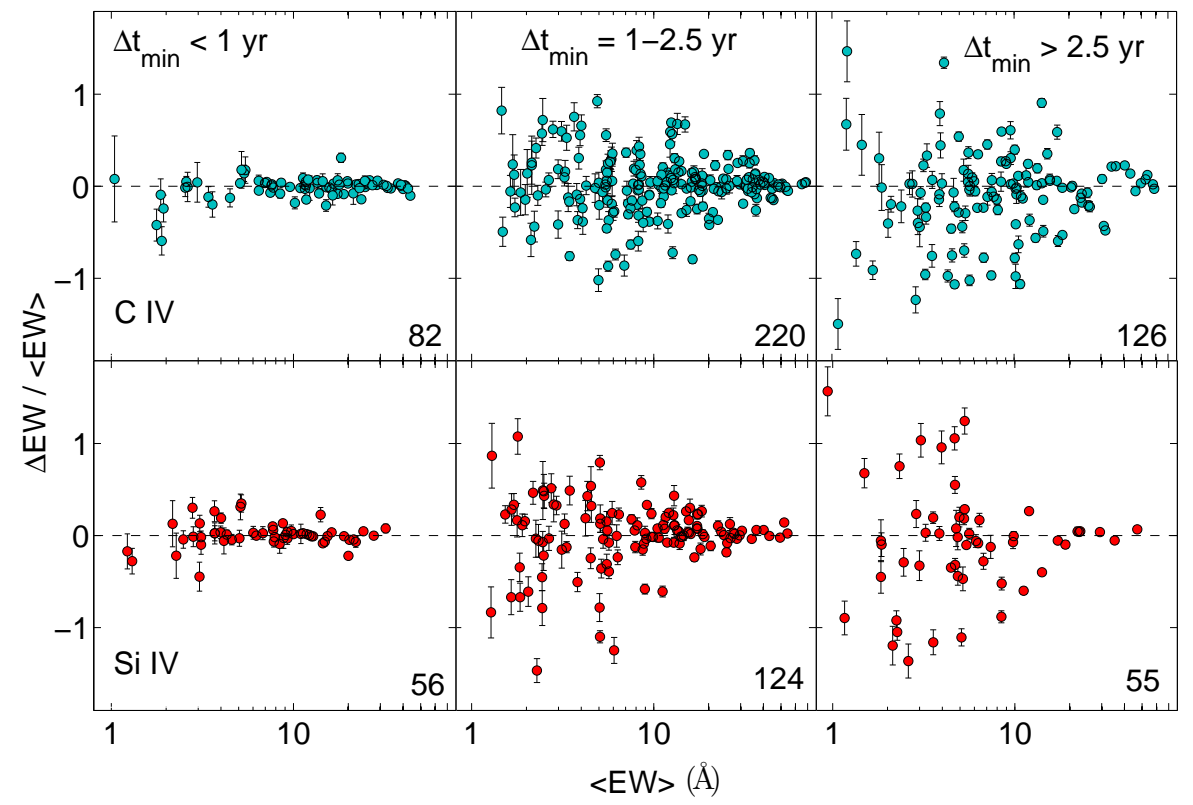

FIG. 16. - Fractional EW variation, $\Delta \mathrm{EW} /\langle\mathrm{EW}\rangle$, vs. average EW over the two relevant epochs, $\langle\mathrm{EW}\rangle$, for C IV (blue) and Si IV (red) BAL troughs for three different timescales as labeled. The $x$-axis is logarithmic. The number of data points are given in the lower right of each panel.

trough depth, $d_{\mathrm{BAL}}$ (defined in Section 3.2), vs. $|\Delta \mathrm{EW}|$ for variations on short, moderate, and long timescales (see Figure 17).

The Spearman test results show significant $(>99.9 \%)$ correlations between $|\Delta \mathrm{EW}|$ and $\Delta v$ for all three timescale ranges indicating that wider BAL troughs tend to vary more than narrower ones; this is perhaps as expected since wider BAL troughs might have a better chance of containing variable regions. Unlike BAL- trough width, we find no significant correlation between $|\Delta \mathrm{EW}|$ and $d_{\mathrm{BAL}}$. However, we do note from Figure 17 that the EWs of the deepest BAL troughs (those with $\left.d_{\text {BAL }}>0.6\right)$ appear to vary less than shallower ones. An even larger sample will be required to investigate this behavior reliably since we have limited trough statistics at large values of $d_{\mathrm{BAL}}$. This apparent behavior could be due to more saturated absorption at large $d_{\mathrm{BAL}}$ values; BAL absorption is often saturated and, due to partial 
covering, saturated BALs are usually found as non-black absorption (e.g., Hamann 1998; Arav et al. 1999).

The $\Delta \mathrm{EW}$ distribution as a function of $d_{\mathrm{BAL}}$ suggests that shallow BAL troughs on average increase their EWs and deep BAL troughs on average decrease their EW on moderate and long timescales (see Figure 17). We search for a correlation between $\Delta \mathrm{EW}$ and $d_{\mathrm{BAL}}$ using the Spearman test and find likely correlations for both moderate and long timescales $(P=2.6 \%$ and $P=5.6 \%$, respectively). To show this trend, we also compare the mean $\Delta \mathrm{EW}$ values for $\mathrm{BAL}$ troughs that are deeper and shallower than the median BAL trough depth. The median BAL-trough depth is 0.32 for moderate timescales and 0.31 for long timescales. We found the mean $\triangle \mathrm{EW}$ values to be $0.95 \pm 0.30 \AA$ for BAL troughs with $d_{\mathrm{BAL}}<0.32$ and $-0.42 \pm 0.34 \AA$ for $\mathrm{BAL}$ troughs with $d_{\mathrm{BAL}}>0.32$ on moderate timescales. Similarly, for variations on long timescales the mean $\triangle \mathrm{EW}$ values are $0.94 \pm 0.29 \AA$ and $-0.32 \pm 0.36 \AA$ for BAL troughs shallower and deeper than $d_{\mathrm{BAL}}=0.31$, respectively. These results indicate that the statistical variation of a BAL trough depends not just on its EW and on the timescale between spectra, but also on its depth.

It has been found in previous studies that weak BAL troughs generally can achieve higher velocities than strong BAL troughs (e.g., Gibson et al. 2009; Capellupo et al. 2011; Filiz Ak et al. 2012); our data also show this trend. Considering our results indicate that weak BAL troughs tend to have smaller EW variations, we search for correlations between $|\Delta \mathrm{EW}|$ and central velocity of a BAL trough, $v_{\text {cent }}$ (see Section 3.2 for definition). Figure 17 displays $\triangle \mathrm{EW}$ for $\mathrm{C}$ IV BAL troughs as a function of $v_{\text {cent }}$ for variations on three different timescales. Spearman test results show no significant correlations for variations on short, moderate, and long timescales.

BAL variability has been noted to be fractionally stronger among weaker troughs (e.g., Lundgren et al. 2007; Gibson et al. 2008; Capellupo et al. 2011). However, since the average outflow velocity is higher for the population of weak BAL troughs than for strong troughs, this finding could, in principle, ultimately be a velocity effect. Owing to limited sample sizes, it has been difficult to assess if trough weakness or trough velocity is the primary driver of increased fractional BAL variability (e.g., Capellupo et al. 2011; Filiz Ak et al. 2012). To assess this with our larger sample, in Figure 18 we plot $\Delta \mathrm{EW} /\langle\mathrm{EW}\rangle$ vs. both $\langle\mathrm{EW}\rangle$ and $v_{\text {cent. Spearman }}$ test results show that there is a clear correlation between $|\Delta \mathrm{EW} /\langle\mathrm{EW}\rangle|$ and $\langle\mathrm{EW}\rangle$ (significance level of $>99.9 \%$ ), but no significant correlation between $|\Delta \mathrm{EW} /\langle\mathrm{EW}\rangle|$ and $v_{\text {cent }}$. Therefore, our results establish that trough weakness is indeed the primary driver of increased fractional BAL variability.

We also search for correlations between $|\Delta \mathrm{EW}|$ and $\Delta v, d_{\mathrm{BAL}}$, and $v_{\text {cent }}$ for Si IV BAL troughs on three different timescales using Spearman tests. The test results show highly significant $(>99.9 \%)$ correlations between $|\Delta \mathrm{EW}|$ and $\Delta v$ for Si IV BAL troughs on all three timescales. Similar to the results for C IV BAL troughs, we found no significant correlation between $|\Delta \mathrm{EW}|$ and $d_{\mathrm{BAL}}$ or $|\Delta \mathrm{EW}|$ and $v_{\text {cent }}$ for Si IV BAL troughs.

\subsection{Comparison of $C$ IV vs. Si IV EW Variations}

Owing to the differences in ionization potentials (45.1 eV for Si IV and $64.5 \mathrm{eV}$ for C IV), abundances, and consequently optical depths, comparisons of BAL-trough variations between $\mathrm{C}$ IV and Si IV BAL troughs have an important role in assessing possible causes of BAL variations. To investigate variations of $\mathrm{C}$ IV and Si IV BAL troughs from the same absorbing material, we compare EWs and EW variations of BAL troughs of these two ions at corresponding velocities.

As is well known from previous studies (e.g., Weymann et al. 1991; Barlow 1993; Gibson et al. 2009, 2010; Capellupo et al. 2011, 2012), C IV BAL troughs are not always accompanied by SiIV troughs. Therefore, we search for a CIV BAL trough in overlapping velocity ranges for each Si IV BAL trough in two-epoch spectra of each quasar that sample minimum timescales of more than $1 \mathrm{yr}, \Delta t_{\min , 1}$. Given that the Si IV BAL region can be contaminated by other lines between -13000 and $-30000 \mathrm{~km} \mathrm{~s}^{-1}$ (see Section 4.1), in this comparison we search for accompanying C IV BAL troughs for a total of $136 \mathrm{Si}$ IV BAL troughs with $-3000>v_{\text {cent }}>$ $-13000 \mathrm{~km} \mathrm{~s}^{-1}$.

Figure 19 compares the average EW values, $\langle\mathrm{EW}\rangle$, for CIV and Si IV BAL troughs in overlapping velocity ranges. Consistent with previous studies (e.g., Gibson et al. 2009, 2010; Capellupo et al. 2012), we find that Si IV absorption tends to be weaker than C IV absorption present at the same velocities. The vast majority of Si IV BAL troughs ( $\approx 96 \%, 130$ of 136 ) have EWs smaller than the corresponding C IV troughs.

A number of previous studies have searched for correlations between the variations of BAL troughs of different ions. Gibson et al. (2010) studied variations of nine C IV and SiIV BAL troughs. Although they were not able to find any correlation between $\mathrm{EW}$ variations of $\mathrm{CIV}$ and Si IV BAL troughs, they showed a strong correlation for fractional variations (see their Figures 11 and 12). Gibson et al. (2010) showed that fractional variations of Si IV absorption tend to be larger than those of C IV. Capellupo et al. (2012) monitored the C IV and Si IV BAL variability relationship using multi-epoch observations of a sample of 24 quasars. Although they were unable to demonstrate any significant correlation between absolute variations of C IV and Si IV troughs (see their Figures 6 and 7), they noted a weak trend toward greater fractional change for Si IV.

Figure 20 presents comparisons of $\Delta \mathrm{EW}$ and fractional $\triangle \mathrm{EW}$ measurements for $\mathrm{CIV}$ and $\mathrm{Si} I \mathrm{~B}$ BL troughs in overlapping velocity ranges. Consistent with previous studies, the EW variations of CIV and Si IV BAL troughs almost always occur in the same direction, and both the $\Delta \mathrm{EW}$ and $\Delta \mathrm{EW} /\langle\mathrm{EW}\rangle$ of $\mathrm{C}$ IV and Si IV BAL troughs are clearly correlated. The Spearman test results demonstrate that the correlations are highly significant $(>99.9 \%)$ for both measurements. We also found that both the $|\Delta \mathrm{EW}|$ and $|\Delta \mathrm{EW} /\langle\mathrm{EW}\rangle|$ of $\mathrm{C}$ IV and Si IV BAL troughs are strongly correlated (at $>99.9 \%$ significance), indicating that while the BAL troughs almost always vary in the same direction, the magnitudes of the variations are also correlated.

As is apparent from Figure 20, both the $\Delta \mathrm{EW}$ and $\Delta \mathrm{EW} /\langle\mathrm{EW}\rangle$ correlations have significant intrinsic scat- 

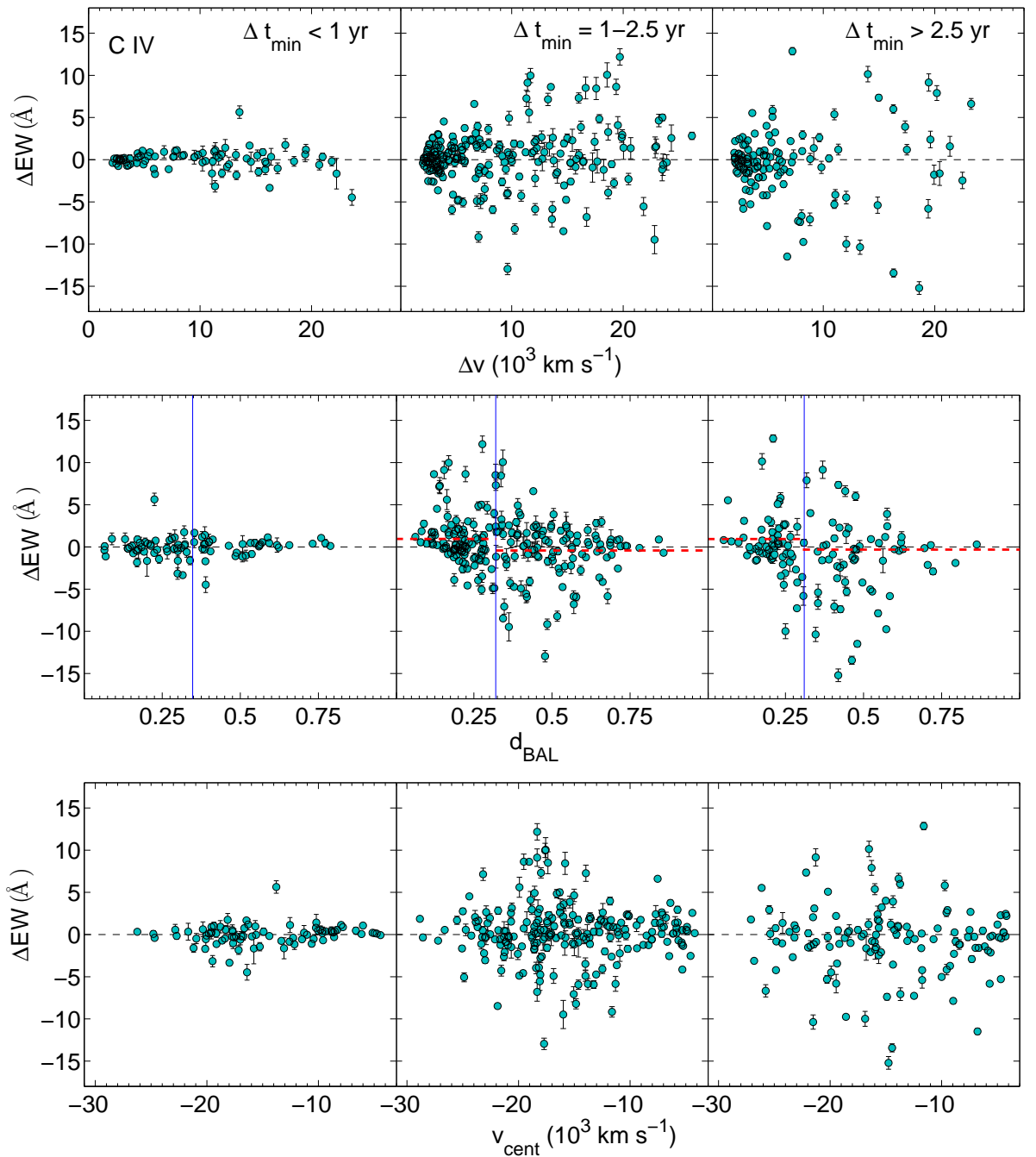

Fig. 17.- C IV BAL-trough EW variation, $\Delta \mathrm{EW}$, as a function of BAL-trough width, $\Delta v$ (top panels), average depth of BAL troughs, $d_{\mathrm{BAL}}$ (middle panels), and centroid velocity, $v_{\text {cent }}$ (bottom panels) for variations on three different timescales as labeled. Vertical blue lines in the middle panels indicate the median $d_{\mathrm{BAL}}$ values and horizontal dashed red lines show the average $\Delta \mathrm{EW}$ values for BAL troughs.

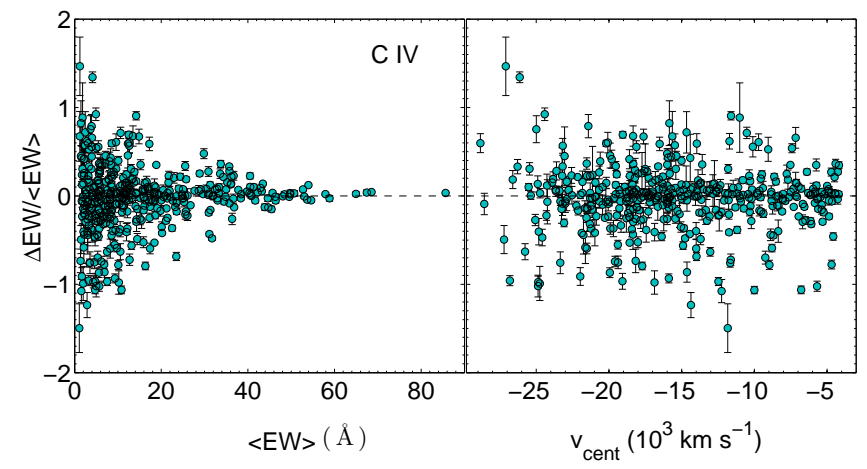

FIG. 18.- C IV BAL-trough $\Delta \mathrm{EW} /\langle\mathrm{EW}\rangle$ as a function of $\langle\mathrm{EW}\rangle$ (left) and $v_{\text {cent }}$ (right) for variations on timescales of more than 1 yr.

ter. To determine the relationship between EW variations of CIV and Si IV BAL troughs, we use a Bayesian linear-regression model that considers the intrinsic scatter of the sample (Kelly 2007). The algorithm estimates regression parameters using random draws from the posterior distribution. We calculate the mean and standard deviation of model parameters that are found using 10000 random draws from our sample. Using the linear-

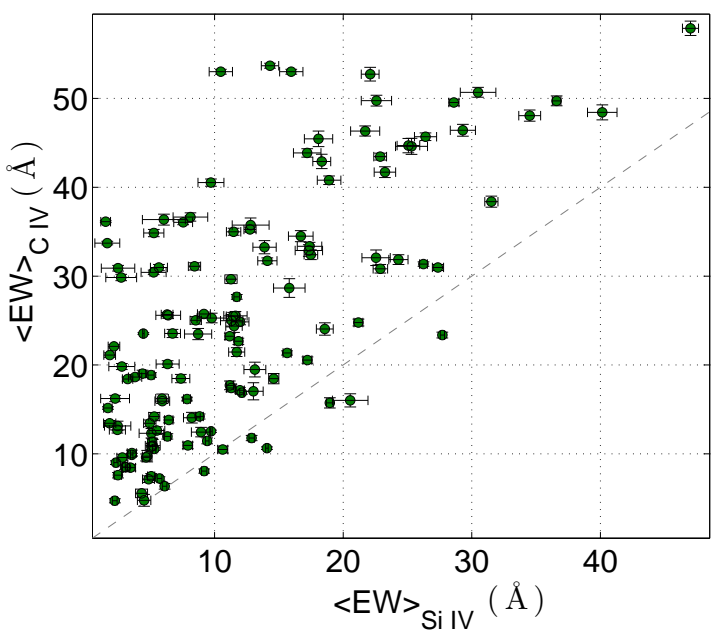

FIG. 19.- Comparison of C IV and Si IV BAL-trough EWs for troughs having corresponding velocities. The dashed line indicates equal strengths of these two absorption troughs. C IV BAL troughs tend to be stronger than Si IV BAL troughs.

regression parameters from this algorithm, we found the 

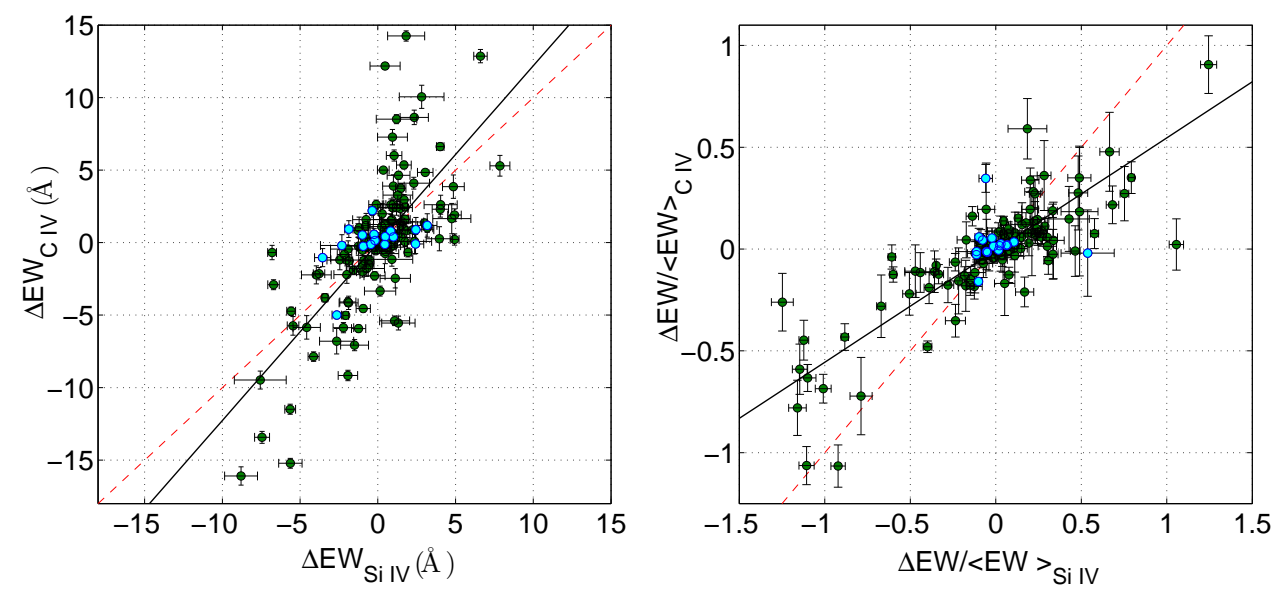

FIG. 20.- Comparison of the variability of C IV vs. Si IV BAL troughs. The left panel compares EW variations for BAL troughs having corresponding velocities, and the right panel compares fractional EW variations. The light blue circles indicate Si IV and C IV BAL troughs with similar EWs as defined in the text. The dashed line in each panel has a slope of unity and zero offset. CIV and Si IV trough variations are clearly correlated; the solid black lines in each panel show the best-fit relations.

following relations:

$$
\begin{array}{r}
\Delta \mathrm{EW}_{\mathrm{CIV}}=(1.223 \pm 0.106) \times \Delta \mathrm{EW}_{\mathrm{Si} \text { IV }} \\
-(0.038 \pm 0.271) \\
\frac{\Delta \mathrm{EW}}{\langle\mathrm{EW}\rangle_{\mathrm{C} \mathrm{IV}}}=(0.551 \pm 0.032) \times \frac{\Delta \mathrm{EW}}{\langle\mathrm{EW}\rangle_{\mathrm{Si} \mathrm{IV}}}
\end{array}
$$

The standard deviation of the intrinsic scatter is $3.034 \AA$ for the $\Delta \mathrm{EW}$ and 0.123 for the $\Delta \mathrm{EW} /\langle\mathrm{EW}\rangle$ distributions between C IV and Si IV BAL troughs.

Equation 8 shows that the slope of the relationship between $\triangle \mathrm{EW}$ for $\mathrm{C}$ IV and $\mathrm{Si} I V \mathrm{BAL}$ troughs is close to unity. The dispersion of the data points increases for large variations of C IV BAL troughs, indicating a possible non-linear component of correlation, especially for $\left|\Delta \mathrm{EW}_{\mathrm{CIV}}\right| \gtrsim 10 \AA$. The $\Delta \mathrm{EW} /\langle\mathrm{EW}\rangle$ relation between CIV and Si IV BAL troughs shows that the fractional $\mathrm{EW}$ variation of CIV BAL troughs is about half of the fractional EW variation of Si IV BAL troughs at a corresponding velocity. This result is consistent with the findings of Gibson et al. (2010) and Capellupo et al. (2012).

In Section 4.5, we showed that weak BAL troughs tend to have larger fractional EW variability compared to strong BALs. In order to assess if the fractional EW of Si IV BAL troughs is more variable because, in general, Si IV BAL troughs are weaker than C IV BAL troughs (see Figure 19), we examine Si IV and C IV BAL troughs with similar EWs. We select a subset of BAL troughs where $0.8<\mathrm{EW}_{\mathrm{Si} I V} / \mathrm{EW}_{\mathrm{CIV}}<1.2$ (a total of 17 cases) and repeat the linear-regression modeling. Analogous to Equation 8 , we found the relation between $\triangle \mathrm{EW}$ for these similar-strength $\mathrm{C}$ IV and Si IV BAL troughs to be $\Delta \mathrm{EW}_{\mathrm{C} \text { IV }}=(0.486 \pm 0.267) \times \Delta \mathrm{EW}_{\mathrm{Si} \mathrm{IV}}+(0.159 \pm 0.413)$, indicating that Si IV troughs vary more in EW compared to C IV troughs of matched EW. Unfortunately, however, we could not derive a useful analog to Equation 9 since the fitted slope was poorly constrained by the data, and thus further work is needed to address this matter.

Physically, we do expect the fractional variations of Si IV to be usually equal to or larger than those of C IV. BAL troughs have lower column densities and covering factors in Si IV than in C IV (e.g., Baskin et al. 2013). This situation arises primarily because the peak ionization fraction of Si IV is a factor of 2.5 lower than that of $\mathrm{C}$ IV and occurs at a slightly lower ionization parameter (e.g., Figure 4 of Hamann et al. 2000). Thus, C IV and Si IV absorption do not arise from exactly the same parts of an absorbing structure. When an absorbing cloud has a column high enough for the ionization inside it to decrease to the level where CIV is found, the cloud will produce CIV absorption; when the cloud has an even higher column, then both C IV and Si IV absorption will be produced. As a consequence, C IV and Si IV absorption are likely to be on parts of their curves of growth where we see either EW changes in CIV with no detectable Si IV absorption, small EW changes in saturated CIV and large EW changes in unsaturated Si IV (i.e., larger fractional change in SiIV), or small EW changes in both C IV and Si IV due to saturation (equal fractional changes). The foregoing reasoning applies regardless of the origin of variations in C IV and Si IV absorption along the line-of-sight. Our data do rule out a scenario where all trough variations are due to the motion across the line-of-sight of clouds of uniform optical depth.

\subsection{Coordination of $E W$ Variations in BAL Quasars with Multiple Troughs}

Quasars with multiple BAL troughs of the same ion provide an opportunity to investigate connections between distinct BAL troughs at different velocities. Using observations of BAL quasars with multiple troughs, Filiz Ak et al. (2012) demonstrated that variations in the EWs of multiple C IV BAL troughs are strongly correlated; when one BAL trough in a quasar spectrum disappears the other troughs present usually (11 out of 12 BAL troughs) weaken even for velocity offsets as large as $10000-15000 \mathrm{~km} \mathrm{~s}^{-1}$. Consistent with this, Capellupo et al. (2012) show that BAL variations at different velocities in the same ion almost always show coordinated variations.

For the purpose of investigating coordinated variations of multiple BAL troughs of the same ion, we select 107 quasars with multiple CIV BAL troughs from our main sample. We compare EW variations of the lowest-velocity BAL trough with those of the other BAL 

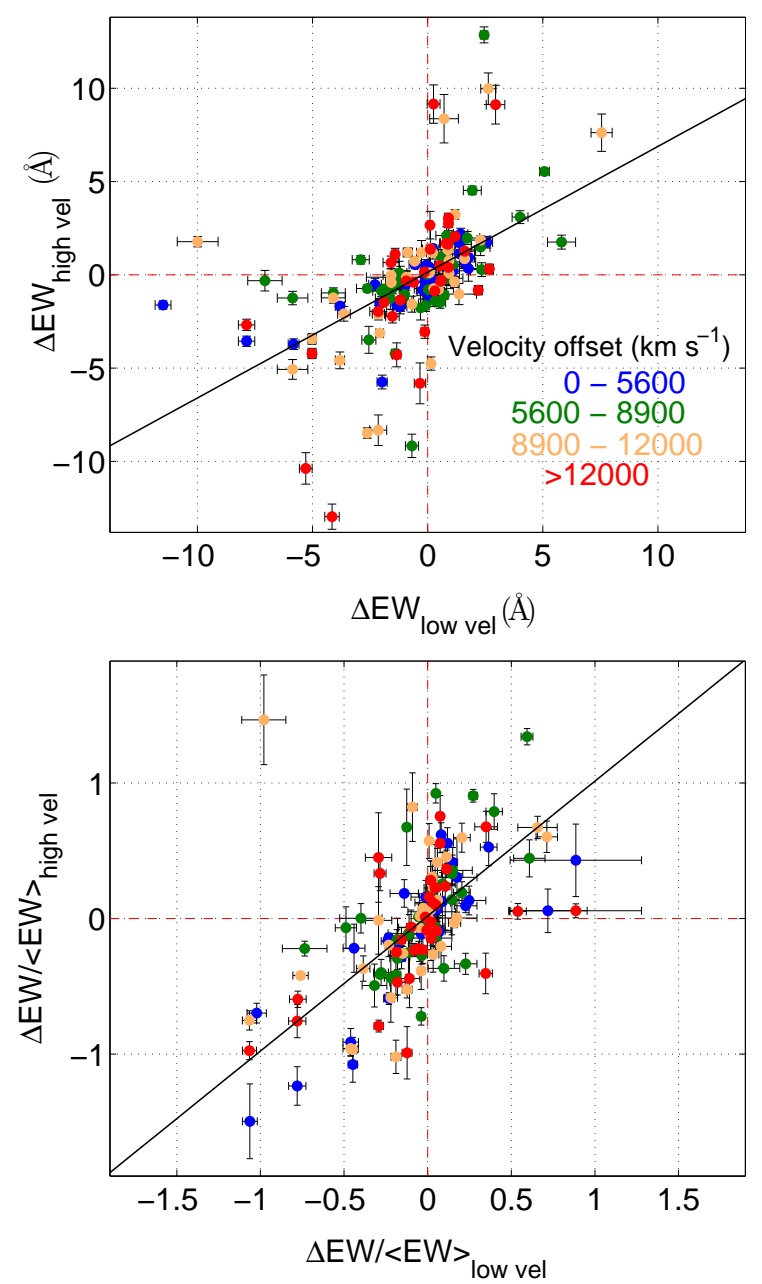

FIG. 21.- Comparison of EW variations (upper panel) and fractional EW variations (lower panel) of distinct troughs for BAL quasars with multiple C IV troughs for variations on timescales of more than $1 \mathrm{yr}$. Colors indicate the velocity separation between troughs; the relevant velocity ranges have been chosen to provide an equal number of data points in each range. The solid black lines in each panel show the best-fit relations. Variations of distinct troughs are correlated for $\mathrm{C}$ IV, although there is substantial scatter in the correlations.

troughs (i.e., BAL troughs at higher velocities) present in the same pair of spectra. We detect a total of 137 highervelocity BAL troughs in the spectra of these 107 quasars. Figure 21 shows the EW variation of the lowest-velocity BAL trough, $\Delta \mathrm{EW}_{\text {low vel }}$, as a function of $\mathrm{EW}$ variations for higher-velocity distinct BAL troughs, $\Delta \mathrm{EW}_{\text {high vel }}$, for variations on timescales of more than $1 \mathrm{yr}$. Similarly, we compare fractional $\mathrm{EW}$ variations for the lowest and higher-velocity BAL troughs in Figure 21. Variations of distinct troughs are correlated, although there is substantial scatter in the correlations. The Spearman test shows that both of the apparent correlations for CIV BAL troughs are highly (>99.9\%) significant.

In order to assess correlations for magnitudes of $\Delta \mathrm{EW}$ and $\Delta \mathrm{EW} /\langle\mathrm{EW}\rangle$ between the lowest-velocity and highervelocity BAL troughs, we run the Spearman test again using the absolute values of the measurements and found highly significant $(>99.9 \%$ ) correlations.

To quantify the relationship between $\mathrm{EW}$ variations of the lowest-velocity and higher-velocity BAL troughs, we use the Bayesian linear regression model of Kelly (2007), considering the intrinsic scatter of the sample. Using this algorithm, we found the following relations:

$$
\begin{array}{r}
\Delta \mathrm{EW}_{\text {high vel }}=(0.674 \pm 0.094) \times \Delta \mathrm{EW}_{\text {low vel }} \\
+(0.141 \pm 0.246) \\
\begin{aligned}
\frac{\Delta \mathrm{EW}}{\langle\mathrm{EW}\rangle_{\text {high vel }}=(0.996 \pm 0.103) \times \frac{\Delta \mathrm{EW}}{\langle\mathrm{EW}\rangle_{\text {low vel }}}} \\
+(0.018 \pm 0.031)
\end{aligned}
\end{array}
$$

The standard deviation of the intrinsic scatter is $2.769 \AA$ for the $\Delta \mathrm{EW}$ and 0.324 for the $\Delta \mathrm{EW} /\langle\mathrm{EW}\rangle$ distributions for multiple C IV BAL troughs.

Equation 10 indicates that BAL troughs at higher velocities tend to have smaller EW variations compared to the lowest-velocity BAL troughs. This result is expected considering that small-EW BAL troughs can achieve higher velocities, and they also tend to have small $\Delta \mathrm{EW}$ values (see Section 4.5 and Figure 15). The slope of Equation 111 is close to unity, indicating that multiple BAL troughs present in the same two-epoch spectra show similar fractional EW variations. This result is even more interesting given that weak BAL troughs tend to have larger $\Delta \mathrm{EW} /\langle\mathrm{EW}\rangle$ compared to strong ones (see Section 4.5 and Figure 16).

Owing to their large fractional variations, disappearing or emerging BAL troughs (see Section [5.3) stand out in the lower panel of Figure 21, The higher-velocity BAL trough of the quasar SDSS J143948.06+042112.8, an emergence candidate with $\Delta \mathrm{EW} /\langle\mathrm{EW}\rangle_{\text {high vel }}=1.46$, is found as an outlier at the top-left of the plot. The other emergence candidate, the higher-velocity BAL trough of the quasar SDSS J151312.41+451033.9, is found at the extreme of the correlation with $\Delta \mathrm{EW} /\langle\mathrm{EW}\rangle_{\text {high vel }}=1.34$. Similarly, the higher-velocity BAL trough of SDSS J095901.24+550408.2 that satisfies the disappearance criteria used in Filiz Ak et al. $(2012)$ is shown at the lower-left of the plot $\left(\Delta \mathrm{EW} /\langle\mathrm{EW}\rangle_{\text {high vel }}=-1.5\right)$.

Table 6 presents the number of additional BAL troughs that vary in the same or opposite direction as the lowestvelocity BAL trough. We found that $78.1 \pm 8.3 \%$ of the additional CIV BAL troughs show correlated variations with the lowest-velocity BAL trough. A combinatorial probability calculation shows that the probability of obtaining such a result for independently varying BAL troughs is $\approx 10^{-12}$. Assuming that $\mathrm{C}$ IV BAL trough pairs show a mixture of perfectly correlated and perfectly uncorrelated variations, then some apparently correlated variations will in fact be uncorrelated variations that appear correlated just by chance. Accounting for those cases using Table 6, the fraction of C IV BAL troughs with variations arising from a mechanism correlated between troughs is $77 / 137$, or $56.2 \pm 7.2 \%$.

To determine the velocity range over which coordinated variations of multiple troughs occur, we calculate the velocity offset of those BAL troughs at higher velocities from the lowest-velocity BAL trough present in the same pair of spectra. Figure 22 shows the percentage of additional C IV BAL troughs at a given velocity offset with increasing strength $(\Delta \mathrm{EW}>0)$ and decreasing 
strength $(\Delta \mathrm{EW}<0)$ in comparison with the $\Delta \mathrm{EW}$ variation of the lowest-velocity BAL trough. We find that even BAL troughs separated from the lowest-velocity trough by $15000-20000 \mathrm{~km} \mathrm{~s}^{-1}$ show generally correlated variations. Figure 21 indicates that BAL troughs with small velocity offsets plausibly have similar scatter about the correlations as troughs with large velocity offsets. To assess if troughs with small velocity offsets show better coordination than those with large velocity offsets, we investigate the fraction of BAL troughs showing coordinated variations as a function of velocity offset using the velocity ranges given in Figure 21. We found that the fraction of higher-velocity BAL troughs showing coordinated variations is constant at $\approx 78 \%$ within the error bars, indicating no strong dependence upon velocity offset.

We also examine the coordination of multiple troughs for the Si IV transition. Similar to C IV BAL troughs, the $\Delta \mathrm{EW}$ and $\Delta \mathrm{EW} /\langle\mathrm{EW}\rangle$ variations for Si IV are correlated; the Spearman test indicates that the significance level is $>99.9 \%$ for both correlations. As for C IV BAL troughs, multiple Si IV BAL troughs also tend to vary in the same direction. The Si IV BAL-trough region, however, is subject to contamination by some other lines in the velocity range of $13000-30000 \mathrm{~km} \mathrm{~s}^{-1}$ (see Section 4.1). Considering such contamination, we do not perform further examinations for multiple Si IV troughs.

\subsection{EW Variations as a Function of Quasar Properties}

In this section, we study BAL-trough EW variations as a function of quasar properties, including luminosity, Eddington luminosity ratio, redshift, and radio loudness.

\subsubsection{Luminosity}

Previous studies (e.g., Kaspi et al. 2005; Bentz et al. 2009) have investigated the relationship between luminosity and broad-line region (BLR) size in AGN. In these studies the derived power-law relation indicates that the size of the BLR increases with increasing quasar luminosity. Given that the BAL region often lies outside the BLR (e.g., BALs often absorb BLR emission), this relation may also be indicative of an increasing size and decreasing orbital (transverse) velocity of the BAL region with increasing luminosity. A dependence of the transverse BAL velocity, and/or continuum source region size, on luminosity can potentially change the variability of BAL outflows as a function of luminosity. Furthermore, Laor \& Brandt (2002) found a correlation between outflow velocity and quasar luminosity, indicating that BAL troughs in luminous quasar spectra can achieve higher velocities. Later, a study by Ganguly et al. (2007) investigated outflows and the physical properties of quasars using a sample of 5088 quasars. In addition to finding consistent results with Laor \& Brandt (2002) on the luminosity-outflow relation, Ganguly et al. (2007) showed that the fraction of BAL quasars (relative to all quasars) increases with increasing luminosity. Therefore, we investigate BAL-trough EW variations as a function of the quasar bolometric luminosity, $L_{\mathrm{Bol}}$. As mentioned in Section $2.2 L_{\mathrm{Bol}}$ values are taken from Shen et al. (2011).

In Figure 23, we show the dependence of $\Delta \mathrm{EW}$ for C IV and Si IV BAL troughs upon $L_{\text {Bol }}$ for variations on three different timescales. In each panel of Figure 23, we show standard-deviation curves calculated using a sliding window for luminosity-ordered data points where the mean EW error in each window is statistically removed from the curve. The number of data points contained in each window is given in the lower left of each panel of Figure 23. If BAL variability decreased with luminosity, one would expect to see a larger scatter of the data points at low luminosities as a result of EW variations in both directions (and vice versa for increasing variability with luminosity). In Figure 23, the standard deviation curves for C IV and Si IV on moderate and long timescales may be indicative of BAL variability decreasing with luminosity.

To assess formally any correlation between $L_{\mathrm{Bol}}$ and $|\Delta \mathrm{EW}|$, we use the Spearman test. Table 7 lists the test probability results along with the number of data points in each timescale range. Generally, we do not find significant evidence for correlations between $L_{\mathrm{Bol}}$ and $|\Delta \mathrm{EW}|$ or $L_{\mathrm{Bol}}$ and $|\Delta \mathrm{EW} /\langle\mathrm{EW}\rangle|$. The one exception is for C IV troughs on moderate timescales (99.8\% significance); this is also the case where we have the best trough statistics. However, given the number of trials in our correlation tests, we do not regard this one case as strong evidence for luminosity dependence.

\subsubsection{Eddington Luminosity Ratio and SMBH Mass}

Proga \& Kallman (2004) suggested that the presence of radiatively driven disk winds is highly sensitive to the ratio of the quasar bolometric luminosity to the Eddington luminosity, $L_{\mathrm{Bol}} / L_{\mathrm{Edd}}$. Therefore, quasar BAL properties are plausibly expected to depend upon $L_{\mathrm{Bol}} / L_{\mathrm{Edd}}$. Indeed, studies by Laor \& Brandt (2002) and Ganguly et al. (2007) found a correlation between the outflow velocity of quasar winds and $L_{\mathrm{Bol}} / L_{\mathrm{Edd}}$. To assess the effect of $L_{\mathrm{Bol}} / L_{\mathrm{Edd}}$ on the variability of the $\mathrm{BAL}$ region, we investigate correlations between BALtrough EW variations and $L_{\mathrm{Bol}} / L_{\mathrm{Edd}}$. Shen et al. (2011) calculated $L_{\mathrm{Bol}} / L_{\mathrm{Edd}}$ values using the virial black-hole mass which is estimated using the C IV emission line for $z \geq 1.9$ quasars. As is well known (e.g., Shen et al. 2008, 2011; Shen 2013, and references therein), $L_{\mathrm{Bol}} / L_{\mathrm{Edd}}$ calculations using the C IV emission line show a larger scatter compared to other emission lines such as $\mathrm{H} \beta$ and $\mathrm{Mg}$ II. Therefore, uncertainties in the $L_{\mathrm{Bol}} / L_{\mathrm{Edd}}$ measurement may hide any underlying correlations.

Figure 24 shows $\Delta \mathrm{EW}$ for $\mathrm{C}$ IV and Si IV BAL troughs as a function of $L_{\mathrm{Bol}} / L_{\mathrm{Edd}}$ on short, moderate and long timescales. Similar to Figure 23, we show the standarddeviation curves computed with a sliding window in each panel of Figure 24. As for luminosity, if BAL variability decreased (increased) with Eddington luminosity ratio, one would expect to see a larger (smaller) scatter of the data points at low Eddington-normalized luminosities. However, the standard-deviation curves do not indicate such a trend for CIV and Si IV BAL troughs on the sampled timescales.

We run Spearman tests to assess connections between BAL EW variations and $L_{\mathrm{Bol}} / L_{\mathrm{Edd}}$. The test results generally show no evidence of significant correlations between $L_{\mathrm{Bol}} / L_{\mathrm{Edd}}$ and $|\Delta \mathrm{EW}|$ or $L_{\mathrm{Bol}} / L_{\mathrm{Edd}}$ and $|\Delta \mathrm{EW} /\langle\mathrm{EW}\rangle|$ for $\mathrm{C}$ IV and Si IV BAL troughs on the sampled timescales (see Table 7). However, as for luminosity, the small probability for CIV troughs on moderate 


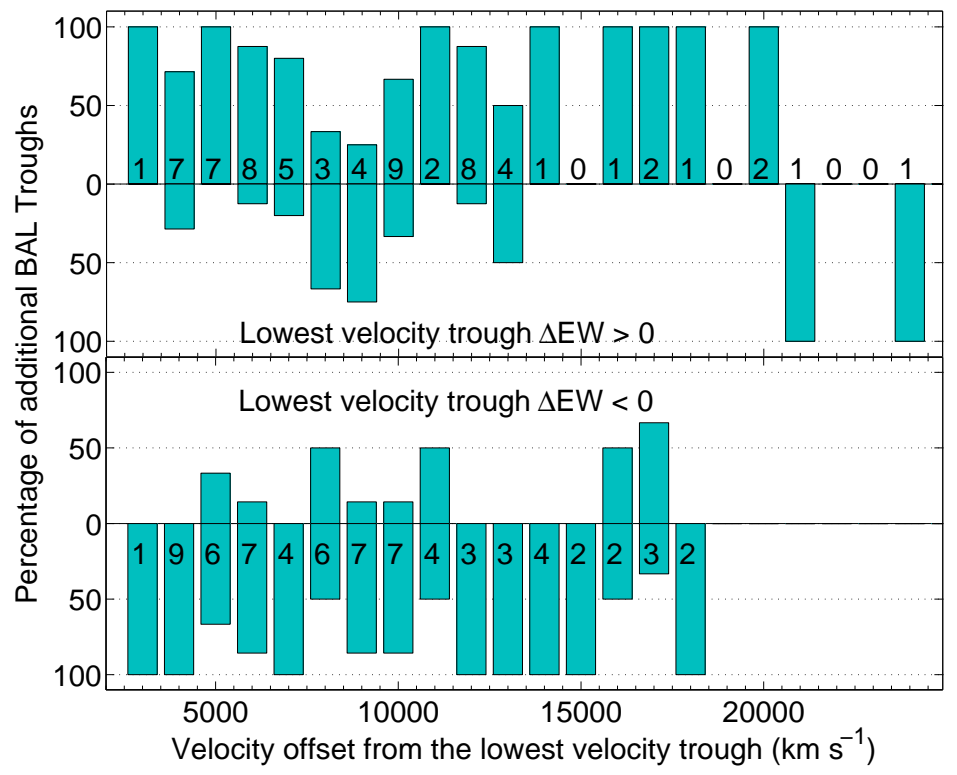

FIG. 22.- Percentage of additional C IV BAL troughs at a given velocity offset with increasing strength (above zero level in each panel; $\Delta \mathrm{EW}>0$ ) and decreasing strength (below zero level; $\Delta \mathrm{EW}<0$ ). The upper panel is for cases where the lowest-velocity BAL trough strengthens $\left(\Delta \mathrm{EW}_{\text {low vel }}>0\right)$, and the lower panel is for cases where it weakens $(\Delta \mathrm{EW}$ low vel $<0)$. The numbers for each bar show the total number of additional BAL troughs found at the relevant velocity offset. Even BAL troughs separated from the lowest velocity trough by $15000-20000 \mathrm{~km} \mathrm{~s}^{-1}$ show generally correlated variations.

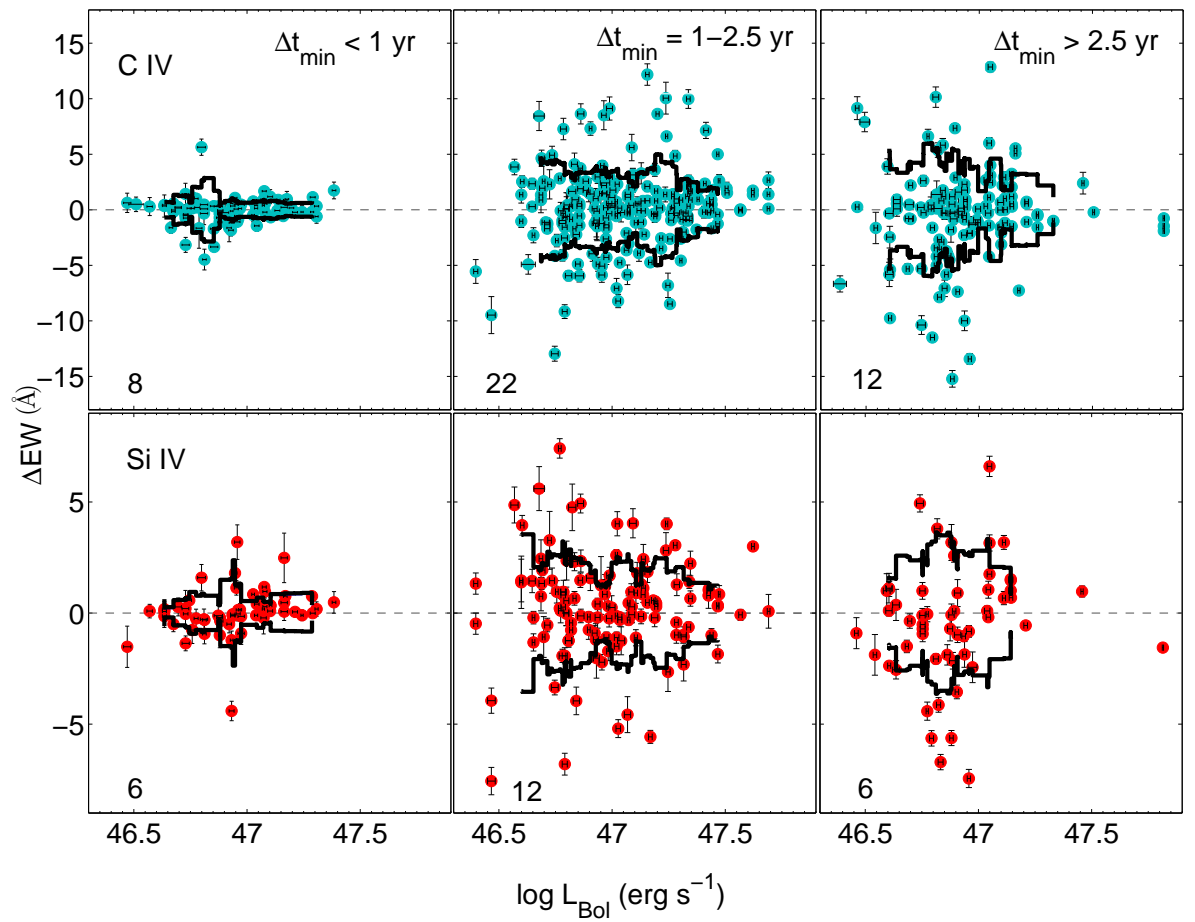

FIG. 23. - EW variability of CIV (upper panels) and Si IV (lower panels) BAL troughs as a function of quasar bolometric luminosity, $L_{\mathrm{Bol}}$, for three different rest-frame timescales as labeled. The black curves in each panel show the running rms computed with a sliding window; the window width, in terms of number of troughs, is given in the lower left of each panel. If BAL variability decreased (increased) strongly with luminosity, one would expect to see a larger (smaller) scatter of the data points at low luminosities. We do not find significant evidence for such behavior on any of the sampled timescales, at least over the order-of-magnitude in luminosity with significant coverage.

timescales might be indicative of a correlation between $L_{\mathrm{Bol}} / L_{\mathrm{Edd}}$ and $|\Delta \mathrm{EW}|$. This result is consistent with the test result between $L_{\mathrm{Bol}}$ and $|\Delta \mathrm{EW}|$ considering the strong relation between $L_{\mathrm{Bol}}$ and $L_{\mathrm{Bol}} / L_{\mathrm{Edd}}$.

In addition, we assess any correlation between EW variations of BAL troughs and SMBH mass estimates from Shen et al. (2011). The Spearman test results show no evidence of significant correlations between $M_{\mathrm{BH}}$ and $|\Delta \mathrm{EW}|$ or $M_{\mathrm{BH}}$ and $|\Delta \mathrm{EW} /\langle\mathrm{EW}\rangle|$ for C IV and Si IV BAL troughs on the sampled timescales (see Table 7). 


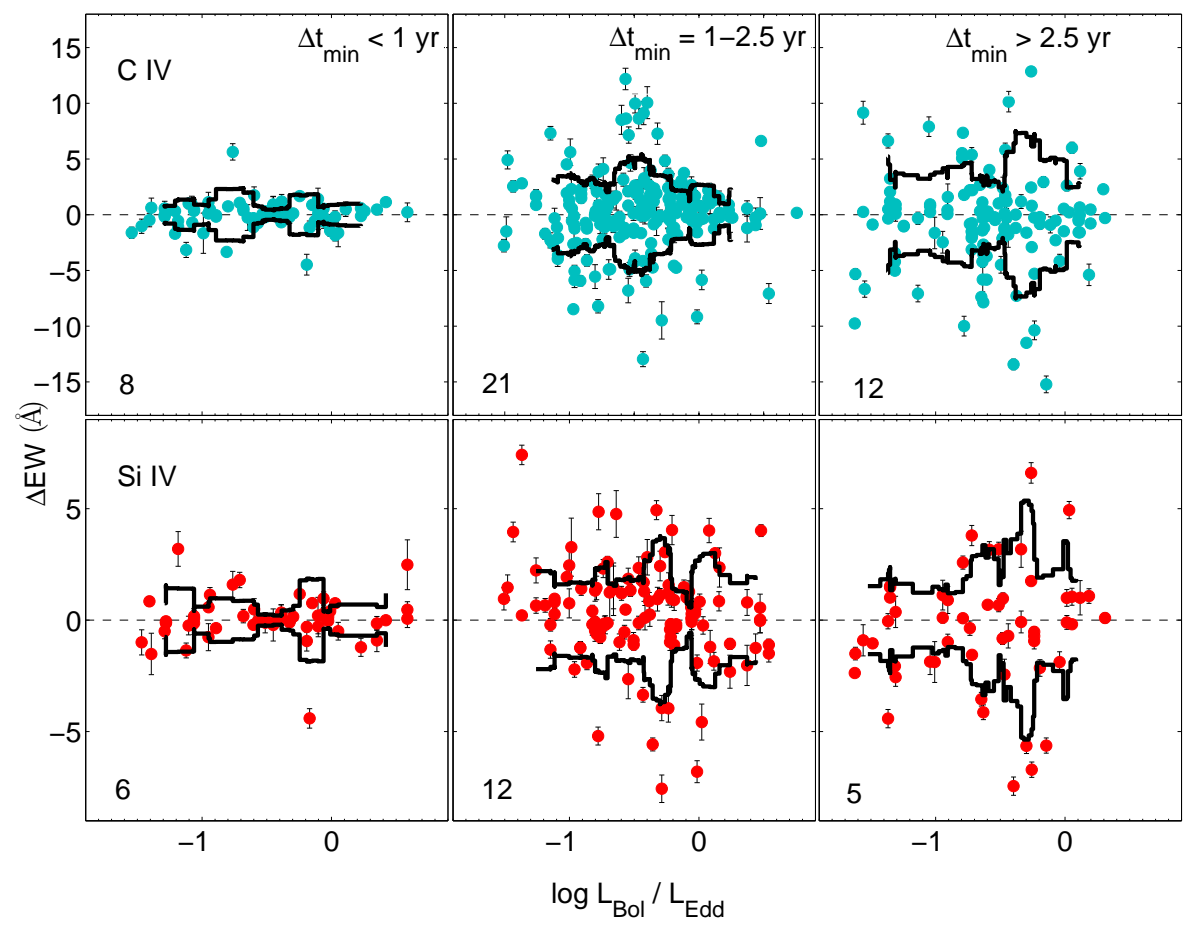

Fig. 24. - Same as Figure 13 but for Eddington-normalized luminosity, $L_{\mathrm{Bol}} / L_{\mathrm{Edd}}$. We do not find any significant evidence for changes in BAL variability with $L_{\mathrm{Bol}} / L_{\mathrm{Edd}}$.

\subsubsection{Redshift}

It is also of interest to assess any dependence of BAL variability upon redshift; e.g., since Allen et al. (2011) reported a relation between the fraction of BAL quasars and redshift. Therefore, we investigate correlations between quasar redshifts and EW variations on short, moderate, and long timescales. Plots similar to Figures 23 and 24 generally do not show significant relations with redshift, consistent with the results in Table 7.

\subsubsection{Radio Loudness}

Do BAL troughs in radio-quiet and radio-loud quasars vary differently? Given that the presence of powerful radio jets in a quasar appears to affect its wind properties (e.g., Becker et al. 2000; Shankar et al. 2008; Miller et al. 2009, 2012; Welling et al. 2013), different BAL variability might be expected for radio-loud quasars.

To address this issue, we first examine $\Delta \mathrm{EW}$ and $\Delta \mathrm{EW} /\langle\mathrm{EW}\rangle$ as a function of $R$ for BAL variations on timescales of more than $1 \mathrm{yr}$; here $R$ is the radio-loudness parameter calculated by Shen et al. (2011) using VLA FIRST observations (see Section 2.2). Radio emission is detected from 33 of our main-sample quasars, nine of which have $R>100$ and thus are radio-loud. We identified $40 \mathrm{C}$ IV and $28 \mathrm{Si}$ IV BAL troughs in multi-epoch spectra of these 33 quasars. In Figure 25. we show the distribution of $\triangle \mathrm{EW}$ for the $40 \mathrm{C}$ IV BAL troughs as a function of $R$. A Spearman test shows no indication of a significant correlation between $|\Delta \mathrm{EW}|$ and $R$. Similarly, we find no significant correlation between $|\Delta \mathrm{EW} /\langle\mathrm{EW}\rangle|$ and $R$. We repeat the correlation tests for those nine radio-loud quasars with $R>100$ and found no significant correlations.

Second, we compare BAL-trough EW variations on matched timescales for radio-detected $(R \gtrsim 1)$ and radio- non-detected $(R \lesssim 1)$ quasars. We randomly select a sample of $\mathrm{CIV}$ BAL troughs of radio-non-detected quasars that have matching timescales with the $40 \mathrm{C} \mathrm{IV}$ BAL troughs, and then run a two-sample KS test comparing the $\Delta \mathrm{EW}$ distributions of these two samples. The KS test results show no inconsistency between the two $\triangle \mathrm{EW}$ distributions. Similarly, we do not find significant inconsistency from the comparison of the $\Delta \mathrm{EW} /\langle\mathrm{EW}\rangle$ distributions.

We also repeat the matched-sample selection and $\mathrm{KS}$ tests for those nine radio-loud quasars with $R>100$. We find no inconsistency between BAL-trough variability of radio-loud and radio-non-detected quasars from the comparison of the $\Delta \mathrm{EW}$ and $\Delta \mathrm{EW} /\langle\mathrm{EW}\rangle$ distributions for C IV BAL troughs.

\section{DISCUSSION}

In Section 4, we presented our main observational results with generally limited physical discussion. In this section, we therefore examine the physical implications of our most notable findings for quasar winds.

\subsection{The Frequency of BAL Variations}

Our large sample demonstrates that CIV and Si IV BAL variations are common on multi-year rest-frame timescales. About 50-60\% of both C IV and Si IV BALs vary detectably over 1-3.7 yr, with the exact derived fraction depending upon the approach taken in variable BAL-trough identification. The fraction of quasars showing BAL-trough variability is even higher, since some BAL quasars possess more than one trough. Using the two-epoch observations of our sample, we find that $62.2_{-4.6}^{+4.9 \%}(181 / 291)$ and $59.1_{-5.7}^{+6.3 \%}(107 / 181)$ of $\mathrm{BAL}$ quasars show $\mathrm{C}$ IV and Si IV trough variability, respectively. Our results are derived from a much larger sample 


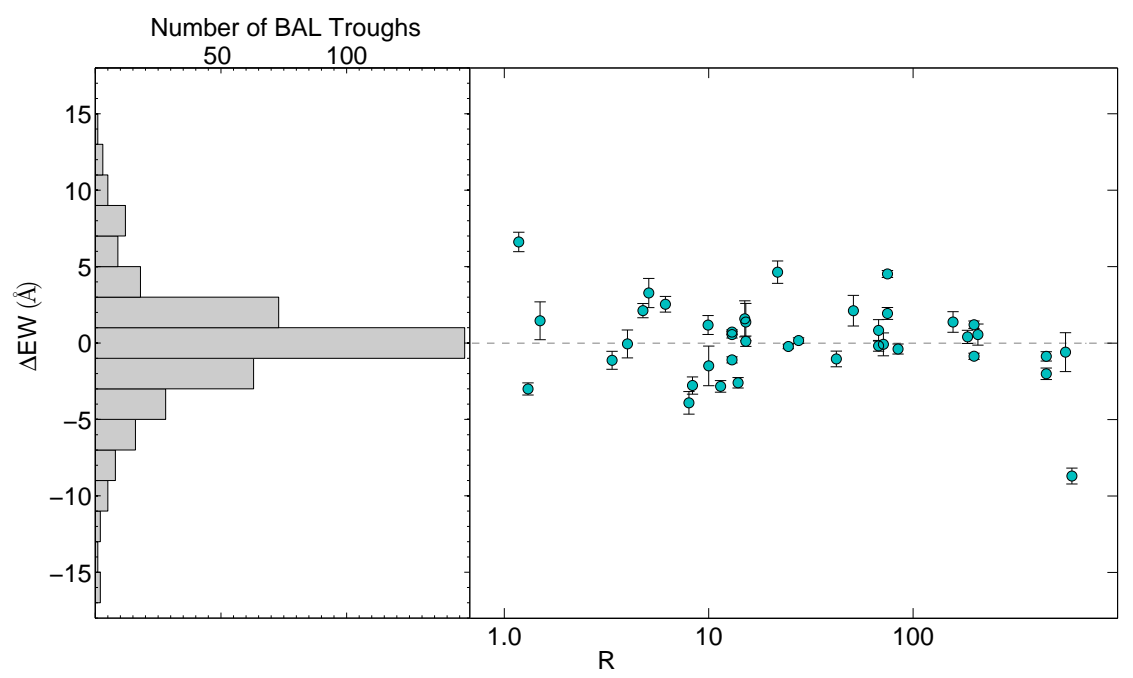

FIG. 25.- EW variation for CIV BAL troughs as a function of radio-loudness parameter, $R$, for variations on timescales of more than 1 yr. Individual data points are plotted for quasars with radio detections in the FIRST catalog, while a histogram is shown along the left-hand side for quasars lacking radio detections. We do not find strong evidence for changes in BAL variability with $R$.

of quasars than past work, and they also do not suffer from systematic uncertainty owing to multi-counting biases (see Footnote 19) or preferential observation of BAL quasars known to show trough variability (e.g., see Section 3.3 of Capellupo et al. 2013). However, our results are generally consistent with past studies. For example, Capellupo et al. (2013) found that $\approx 55 \%$ of their sample of BAL quasars showed C IV trough variability on a timescale of 2.5 yr (see their Figure 12). Gibson et al. (2008) found that 12 of their $13(\approx 92 \%)$ BAL quasars showed C IV trough variability, although they sampled longer timescales of 3-6 yr where a higher percentage of variable BAL quasars is expected.

The high incidence of BAL variability that we find on multi-year timescales is generally supportive of models where most BAL absorption arises within about an order-of-magnitude of the radial distance of the launching region; i.e., 10-1000 light days (see Section 1). However, our results are still consistent with a significant fraction of BAL absorption arising on larger scales. Indeed, some of the troughs in our sample remain remarkably stable over multiple years in high-quality spectra (e.g., see Figure 26). This result is consistent with independent findings that some BAL absorption arises on

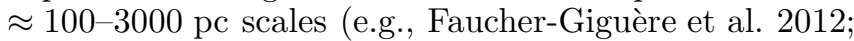
Arav et al. 2013; Borguet et al. 2013), although lack of variability does not strictly require that the absorption arises on these size scales. In some cases, we find both remarkably stable as well as variable troughs in the same object (e.g., SDSS J141513.99+365412.2 and SDSS J225608.48+01557.7 in Figure 26), suggesting that BAL absorption may arise over a wide range of size scales. Those BAL troughs believed to be formed on $\approx 100-3000 \mathrm{pc}$ scales should be intensively monitored for variability as an independent check of their distance from the SMBH.

\subsection{Constraints Upon BAL Lifetimes}

In Section 4.3, we examined the behavior of $\Delta \mathrm{EW}$ and $\Delta \mathrm{EW} /\langle\mathrm{EW}\rangle$ for BAL troughs as a function of rest-frame timescale. One implication of these results is that we can make basic order-of-magnitude estimates of the av- erage "lifetime" of a BAL trough; here the lifetime represents the time over which a BAL trough is seen along our line-of-sight and not necessarily the physical lifetime of the absorbing structure (e.g., a structure could remain intact but move out of our line-of-sight). We use Equation [6]considering that a BAL trough will disappear when the magnitude of its $\mathrm{EW}$ variation is equal to its $\mathrm{EW},|\Delta \mathrm{EW}|=\mathrm{EW}$. In our current sample the measured EWs are in the range $0.3-87.5 \AA$ with a median of $10.9 \AA$. Given that the strengthening and weakening of BAL troughs occur at similar rates (see Section 4.4), solution of Equation 6 for $|\Delta \mathrm{EW}|=10.9 \AA$ gives half of an average BAL lifetime (the other half corresponds to the BAL strengthening from $0 \AA$ to $10.9 \AA$ ). Therefore, this approach reveals that $\left\langle t_{\mathrm{BAL}}\right\rangle=1600_{-900}^{+2100} \mathrm{yr}$. The large error bars of our lifetime estimate largely arise because of the significant difference between the sampled timescales and the derived lifetime. Although the measurements underlying Equation 6 span a factor of $\approx 10^{3}$ in timescale, the lifetime appears a factor of $\sim 10^{3}$ longer still (and thus, of course, the extrapolation used to estimate a lifetime needs to be treated with caution). Our estimate of the average BAL lifetime is consistent with the lower limits for BAL lifetime in Gibson et al. (2008, 2010) and Hall et al. (2011). We note that this average lifetime is long compared to the orbital time of the accretion disk at the expected wind launching radius of 10-100 light days (for a $10^{9} \mathrm{M}_{\odot} \mathrm{SMBH}, t_{\text {orb }}$ is $\sim 50 \mathrm{yr}$ ). It is also long, or at best comparable to, the orbital time at the radius of the BLR of $\approx 1$ light year (e.g., Kaspi et al. $\left.2007 ; t_{\text {orb }} \sim 500 \mathrm{yr}\right)$.

One should keep in mind that BAL lifetimes derived with the above approach depend significantly upon EW, and thus the lifetimes derived here are in agreement with those estimated by Filiz Ak et al. (2012) using a qualitatively different approach. Using a sample of 21 examples of disappearing BAL troughs, Filiz Ak et al. (2012) found the average BAL lifetime to be about a century; the measured EWs of these 21 BAL troughs in the first-epoch spectra were $2.2-10.6 \AA$, with a median of $4.7 \AA$. Applying the approach based upon Equation 6 . 

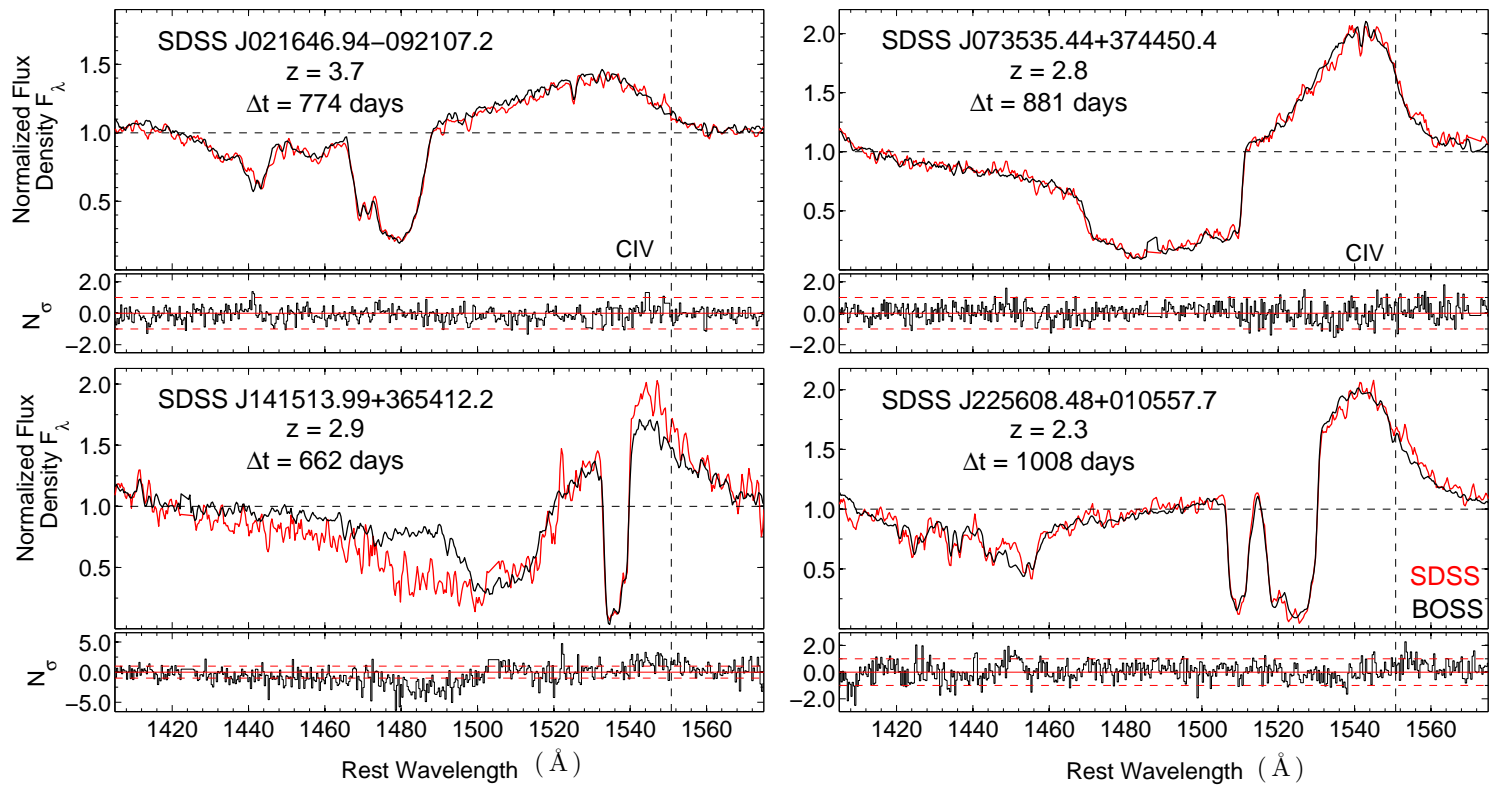

FIG. 26. - Two-epoch spectra from SDSS (red) and BOSS (black) of quasars with BAL troughs that remain remarkably stable over multiple years in the rest frame; in some cases additional distinct BAL troughs that vary are present as well. These are examples of quasars that may have some BAL absorption arising on large physical scales. The dashed vertical lines show the $\mathrm{C}$ IV emission-line rest wavelength. The lower section of each panel shows $N_{\sigma}$ values for SDSS vs. BOSS observations where the dashed red lines show the $\pm 1 \sigma$ level.

we find $\left\langle t_{\mathrm{BAL}}\right\rangle=60_{-22}^{+38} \mathrm{yr}$ for these 21 disappearing $\mathrm{BAL}$ troughs, consistent with Filiz Ak et al. (2012).

\subsection{Relation of BAL Disappearance and Emergence to General BAL Variability}

In this section, we assess where BAL disappearance and emergence events lie within the distribution of BAL EW variability. Since our sample-selection criteria (see Section 2.2) limit the redshift, $\mathrm{SN}_{1700}$, and $\mathrm{BI}^{\prime}$ more strictly than in Filiz Ak et al. (2012), our main sample includes only three of the disappearing $\mathrm{C}$ IV BAL troughs described in Filiz Ak et al. (2012). None of these three has a Si IV BAL trough at corresponding velocities at any epoch. Figure 14 displays the three examples of disappearing C IV BAL troughs. Filiz Ak et al. (2012) found that disappearing BAL troughs tend to have relatively small EWs in their first-epoch spectra; the three examples of disappearing $\mathrm{C}$ IV BAL troughs lie between 0 and $-5 \AA$ in the $\triangle \mathrm{EW}$ distribution. In the distribution of fractional EW variations, all three of the disappearing C IV BAL troughs lie at the negative extreme of the distribution, although they are not a distinct population. These examples of BAL disappearance indicate that disappearance is an extreme example of general BAL variability, rather than a qualitatively distinct phenomenon.

We have visually inspected the BAL troughs whose EWs decreased by at least a factor of five between the two epochs (i.e., BAL troughs with $\Delta \mathrm{EW} /\langle\mathrm{EW}\rangle<-1.33)$. Our main sample includes one additional case of C IV BAL disappearance that satisfies the disappearance criteria used in Filiz Ak et al. (2012); this trough, in the quasar SDSS J095901.24+550408.2, is plotted in the upper-right panel of Figure 14 at $\Delta \mathrm{EW} /\langle\mathrm{EW}\rangle=-1.5$. Two of the main-sample quasars (SDSS J092522.72+370544.1 and SDSS J112055.79+431412.5), whose Si IV BAL trough EWs decreased by at least a factor of five between the two epochs, can be classified as candidates for quasars with disappearing Si IV BAL troughs. The corresponding C IV BAL troughs of these two quasars do not disappear.

Visual inspection of the BAL troughs whose EWs increased by at least a factor of five between the two epochs (i.e., BAL troughs with $\Delta \mathrm{EW} /\langle\mathrm{EW}\rangle>1.33)$ shows that two of the C IV BAL troughs (for the quasars SDSS J143948.06+042112.8 and SDSS J151312.41+451033.9) and two of the Si IV BAL troughs (for the quasars SDSS J145045.42-004400.2 and SDSS J160202.40+401301.4) can be classified as candidates for BAL-trough emergence. These emergence events lie at the positive extreme of the distribution of fractional EW variations, but again do not appear to be a distinct population. The detailed analysis of emergence is beyond the scope of this study, but we plan to address this topic in future work.

\subsection{A Random-Walk Model for The Evolution of $B A L$ Troughs}

Modelers of quasar winds have not yet been able to make quantitative predictions of how BAL-trough EWs should evolve over time, and thus it is not possible to use our observational data on this topic to test wind models directly. Therefore, as an alternative, we test and constrain a "toy" model where long-term EW variations occur as a series of discrete events. Assuming that a BAL-trough EW varies by a fixed amount $\delta \mathrm{EW}$ after a fixed time step $\delta t$, we use a simple one-dimensional (unbiased) random-walk model to characterize the EW evolution. Therefore, we assume that over a period of time, $T$, a BAL trough undergoes $n=T / \delta t$ changes to its EW. For the purpose of defining $T$, we select our data to examine BAL-trough EW variations on timescales of 2.0$2.5 \mathrm{yr}$ (see Section 4.4 for the motivation for this interval choice). Therefore, we assume each BAL trough evolved with the same number of steps over a time $T=2.25 \mathrm{yr}$ (i.e., the mean of the sampled timescales in this interval). The $\Delta \mathrm{EW}$ distribution for C IV BAL-trough variations 


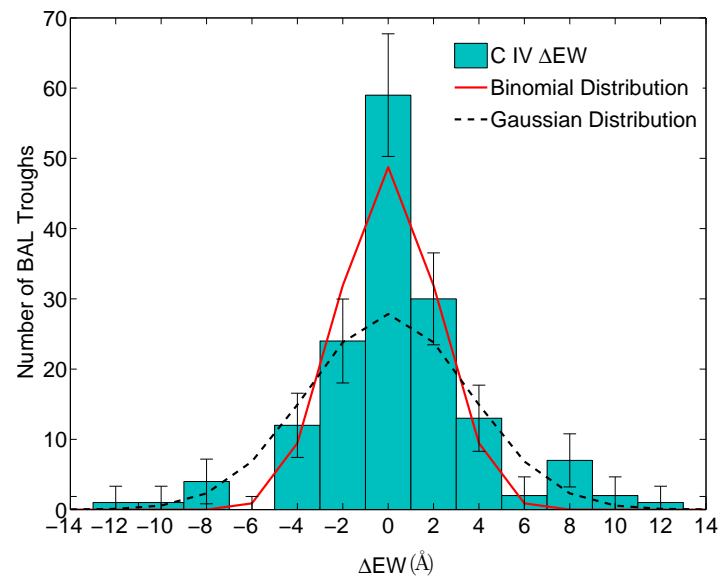

FIG. 27.- Distribution of CIV BAL-trough EW variations, $\triangle \mathrm{EW}$, for variations on timescales of 2.0-2.5 yr and a binomial distribution calculated for a random-walk model with six steps and a step size of $1.65 \AA$. We bin our $\Delta \mathrm{EW}$ data at a bin width of $2 \AA$, and calculate the counting errors following Gehrels (1986). The black dashed line shows the best-matching Gaussian distribution.

\section{on 2.0-2.5 yr timescales is shown in Figure 27}

A random-walk model produces a binomial distribution for $\Delta \mathrm{EW}$ determined by two parameters: the number of steps, $n$, and the size of each step, $s$. To determine the random-walk model that best matches our data, we produced a set of binomial distributions using different numbers of steps and step sizes, and performed a $\chi^{2}$ test between our data and each distribution. A random-walk model with $n=6$ and $s=1.65 \AA$ yields the minimum $\chi^{2}$ for $\mathrm{C}$ IV BAL troughs on timescales of 2.0-2.5 yr. The best-fitting parameters suggest that C IV BAL troughs in this model tend to evolve in a small number of steps on 2.0-2.5 yr timescales. Figure 27 shows the binomial distribution that best matches the $\Delta \mathrm{EW}$ distribution. The $P\left(\chi^{2}, \nu\right)=64.3 \%$ for the bestfitting model indicates that the data are consistent with the random-walk model. We calculate confidence intervals for the model parameters using numerical $\Delta \chi^{2}$ confidence-region estimation for two parameters of interest (see Section 15.6.5 of Press et al. 1992). In Figure 28, we show confidence intervals of $68.3 \%, 90 \%$, and $99 \%$ for two parameters of interest. The best-fitting fixed time step is $\delta t=137_{-53}^{+46}$ days; we calculate uncertainties on this parameter at $68.3 \%$ confidence.

A binomial distribution produced by a random-walk model closely approximates a Gaussian distribution for a large number of steps $(n \gtrsim 20)$; for a smaller number of steps the binomial distribution remains non-Gaussian. Consistent with the fact that the $\Delta \mathrm{EW}$ distribution for C IV BAL troughs on 2.0-2.5 yr timescales is nonGaussian (see Section 4.4), the results in Figure 28 show that our data on the $\triangle \mathrm{EW}$ distribution for $\mathrm{C}$ IV $\mathrm{BAL}$ troughs reject any Gaussian approximation (i.e., large values of $n$ are disfavored). As for the $\Delta \mathrm{EW}$ distributions in Section 4.4, the binomial model distribution also has a stronger central peak compared to the Gaussian approximation.

Similarly, we use a random-walk model to describe the evolution of Si IV BAL-trough EWs. The best-fitting model parameters for Si IV BAL-trough EW variations on timescales of $2.0-2.5 \mathrm{yr}$ are $n=6$ and $s=1.56 \AA$ with

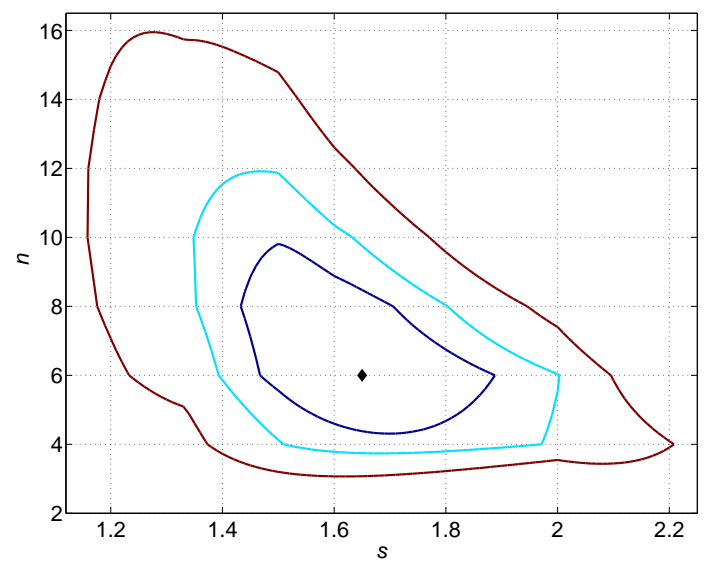

Fig. 28. - 68.3\% (dark blue), 90\% (light blue), and 99\% (dark red) confidence intervals (corresponding to $\Delta \chi^{2}=2.30,4.61$, and 9.21) for the two parameters of the random-walk model describing C IV BAL-trough EW variations, $\triangle \mathrm{EW}$, on timescales of 2.0-2.5 yr. The black diamond indicates the best-fitting model with the number of steps, $n=6$, and the size of each step, $s=1.65 \AA$.

$P\left(\chi^{2}, \nu\right)=25.2 \%$. The best random-walk model parameters for both CIV and Si IV BAL troughs are almost identical; this result is broadly consistent with the correlated C IV and Si IV BAL-trough variations discussed in Section 4.6 .

Using the best-fitting random-walk model $(n=6$, $s=1.65 \AA$ ) calculated for C IV BAL-trough EW variations on timescales of 2.0-2.5 yr, we simulated EW variations for a sample of C IV troughs; we selected the simulated sample to have the same size as our main sample (428). Figure 29 shows a comparison between the simulations and observed EW variations on timescales of 5.9 $\mathrm{hr}$ to $3.7 \mathrm{yr}$. Given that the fixed time step is larger than the shorter timescales sampled by our data, we randomly select the start time of each simulated trough and preserved $\delta t=137$ days for each step. As can be seen in Figure 29, the best-fitting random-walk model on timescales of 2.0-2.5 yr appears to represent acceptably the range of EW variations on a larger range of timescales. We also compared sliding-window standard deviation curves computed for the data and the model. These curves are consistent on timescales of 100-1400 days, and on shorter timescales the data are too limited for proper comparison.

The simple random-walk model examined above should only be considered to be an approximation of a more complex reality; e.g., BAL EWs surely do not evolve via discrete steps after remaining constant for a fixed interval. Nevertheless, the basic apparent success of this model suggests that some of its features are of importance. For example, the derived characteristic timescale of $\approx 137$ days may represent the length of time that BAL EWs tend to increase/decrease monotonically. This timescale also suggests that the distribution of BAL EW values will only approximate a Gaussian for monitoring periods of $\gtrsim 7.5 \mathrm{yr}$ in the rest frame.

\subsection{Shielding Gas Changes as a Driver of Coordinated Multi-Trough Variability?}

Our large sample has allowed us to establish, beyond question, the coordinated variability of multiple troughs in quasar spectra (Capellupo et al. 2012; Filiz Ak et al. 


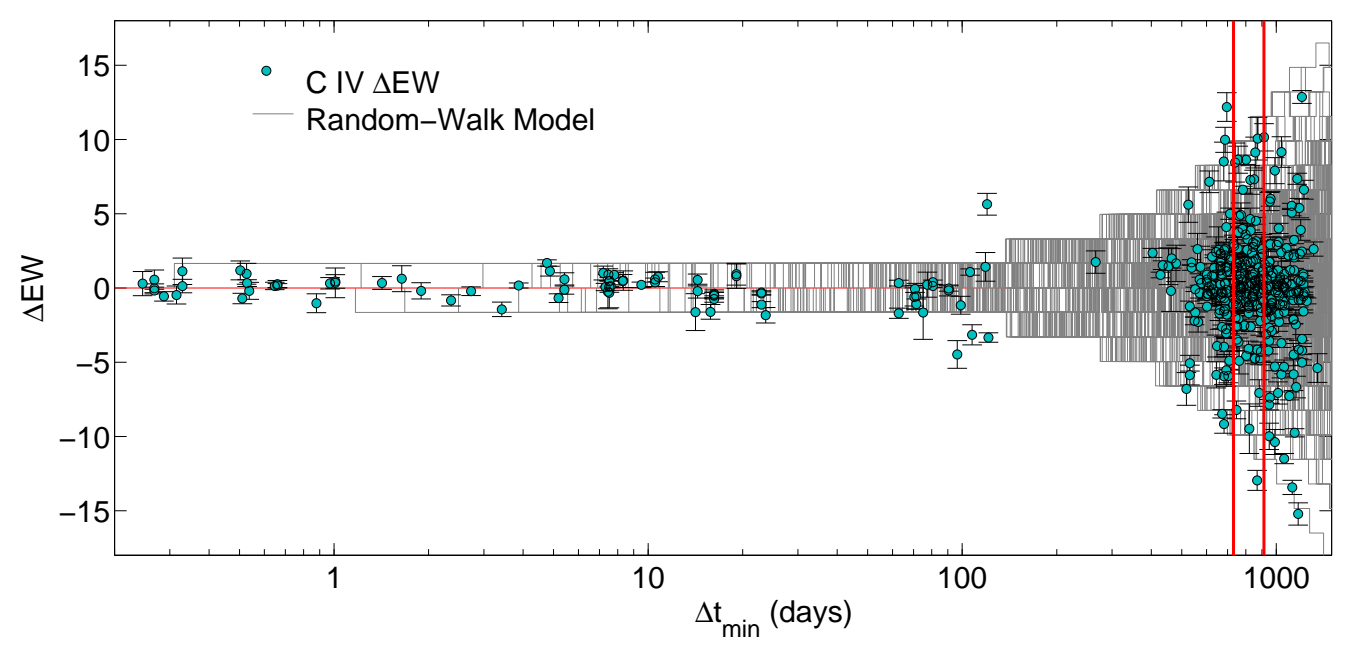

FIG. 29.- Comparison between EW variations for $428 \mathrm{C}$ IV BAL troughs in our main sample and the best-fitting random-walk model. Vertical red bars show a timescale range of $2.0-2.5 \mathrm{yr}$.

2012). This phenomenon is found for both CIV and Si IV troughs; e.g., for C IV the derived probability of this phenomenon being due to chance is $\approx 10^{-12}$. Our investigations of coordinated multi-trough variability show that it is present even for velocity offsets as large as $15000-20000 \mathrm{~km} \mathrm{~s}^{-1}$.

Coordinated multi-trough variability might appear somewhat surprising in the context of quasar-wind models (see Section 10), and it can provide insights into the drivers of BAL variability more generally. Absorption troughs at widely separated velocities should be formed in outflow streamlines that are widely physically separated and thus largely unrelated. Some mechanism capable of acting over a wide range of radii must be present to enforce the coordinated multi-trough variability. Consideration of current quasar-wind models suggests a possible enforcement mechanism is substantial changes in the amount of "shielding gas" along the lineof-sight (e.g., Misawa et al. 2007; Filiz Ak et al. 2012); the shielding gas prevents BAL outflows from being overionized by nuclear extreme UV (EUV) and X-ray photons (e.g., Murrav et al. 1995; Proga 2000). Shieldinggas variability is likely according to quasar-wind simulations (e.g., Proga 2000; Sim et al. 2010, 2012), and it may have been detected in a couple of BAL quasars (e.g., Gallagher et al. 2004; Saez et al. 2012). It can arise due to internal gas motions and/or accretion-disk rotation at the relatively small radii where the shielding gas is located. Changes in the column density of shielding gas can increase or decrease the level of ionizing EUV/X-ray radiation reaching the $\mathrm{BAL}$ wind. In response to this variation, absorption components at different velocities can rise or fall in ionization level together, leading to coordinated multi-trough variations 21 Of course, this same mechanism might be applicable to single-trough BAL quasars as well and thus much of BAL variability in general.

One method for testing this scenario would be to ex-

21 The absorption depth in less-saturated regions of absorption would naturally be more responsive to ionization changes than that in highly saturated regions, and such velocity dependent saturation effects might explain how C IV and Si IV variations usually occur in relatively narrow portions of BAL troughs (see Section 4.2). amine the continuum variability of quasars showing coordinated multi-trough variations. Ideally one would measure the relevant EUV/X-ray ionizing continuum directly, but measurements of the more straightforwardly accessible rest-frame UV continuum might suffice. Unfortunately, limitations in the spectrophotometric calibration of the BOSS spectra do not allow this test to be implemented straightforwardly at present, but it should be possible in the future as BOSS calibration efforts proceed (Margala \& Kirkby 2011; Suzuki 2013).

It is also perhaps possible that EUV/X-ray luminosity variations could primarily drive the BAL variability rather than shielding-gas variations. This would require that some EUV/X-ray radiation is not blocked from reaching the wind by the shielding gas.

\section{SUMMARY AND FUTURE WORK}

We have studied the variability of C IV and Si IV BAL troughs using a systematically observed sample of 291 BAL quasars. We have utilized 699 high-quality spectra of these quasars obtained by SDSS-I/II and BOSS. The main basic observational results of our study are the following:

1. Using a BAL-trough definition designed for the investigation of trough variability in multi-epoch observations, we have identified 428 distinct $\mathrm{C}$ IV and 235 distinct Si IV BAL troughs in the multi-epoch spectra of our 291 "main-sample" quasars. The sampled rest-frame timescales range between $5.9 \mathrm{hr}$ and $3.7 \mathrm{yr}$ with a median of $2.1 \mathrm{yr}$. See Section 3.2

2. We identify variable BAL troughs using two different approaches and find, consistent with earlier work, that BAL variability is common on multiyear timescales. About 50-60\% of both C IV and Si IV BAL troughs detectably vary on rest-frame timescales of 1-3.7 yr. Our large sample size also allows us to quantify the fraction of BALs that vary by a given amount in $\mathrm{EW}$. The cumulative fraction of BAL troughs varying by a given $|\Delta \mathrm{EW}|$ threshold drops with increasing $|\Delta \mathrm{EW}|$, but it remains significant even for $|\Delta \mathrm{EW}|$ values as large as $5 \AA$. See Sections 3.3 and 4.1 
3. C IV BAL variability is found across a wide range of outflow velocity. The percentage of C IV BAL regions showing variability remains relatively constant at $30-40 \%$ at velocities from $-3000 \mathrm{~km} \mathrm{~s}^{-1}$ to $-25000 \mathrm{~km} \mathrm{~s}^{-1}$, and it then rises at higher velocities. The dependence of Si IV BAL variability upon outflow velocity is more difficult to constrain owing to contamination by unrelated emission lines. See Section 4.1 .

4. We show, consistent with earlier work, that C IV and Si IV BAL variations usually occur in relatively narrow portions of BAL troughs (i.e., troughs do not vary monolithically in depth). The variable portions typically span $<30 \%$ of the trough velocity width. Narrow BAL troughs tend to have variable portions that span a larger fraction of their velocity width than broad BAL troughs. See Section 4.2 .

5. The incidence of variability within a BAL trough does not depend significantly upon the relative velocity within the trough. See Section 4.2

6. The EW and fractional EW variations of C IV and Si IV BAL troughs increase with sampled rest-frame timescale, and our large sample allows improved measurement of this effect over a range of $\approx 10^{3}$ in timescale. We find that the rate-of-change of BAL EW is larger on 0.01-1 yr than on 1-3.7 yr timescales. See Section 4.3 .

7. Even in this large sample, the distributions of EW variations and fractional $\mathrm{EW}$ variations for C IV and Si IV BAL troughs show no detectable deviation from symmetry about zero. This indicates that BALs do not strengthen and weaken at significantly different rates. Both the $\triangle \mathrm{EW}$ and $\Delta \mathrm{EW} /\langle\mathrm{EW}\rangle$ distributions appear non-Gaussian. See Section 4.4

8. We have assessed, in several timescale ranges, correlations between BAL EW variations and BALprofile properties, including average EW, velocity width, depth, and outflow velocity. Weak BAL troughs tend to have smaller absolute EW variations but larger fractional EW variations than strong troughs. Decomposing EW into velocitywidth-based and depth-based components, BAL EW variability depends more strongly upon velocity width. We found no correlation between absolute EW variations and trough outflow velocity. See Section 4.5 .

9. The $\Delta \mathrm{EW}$ and $\Delta \mathrm{EW} /\langle\mathrm{EW}\rangle$ values of $\mathrm{C}$ IV $\mathrm{BAL}$ troughs correlate with those of SiIV troughs corresponding in velocity. The $\Delta \mathrm{EW}$ correlation has significant scatter, perhaps suggesting a non-linear correlation. The $\Delta \mathrm{EW} /\langle\mathrm{EW}\rangle$ correlation shows that the fractional $\mathrm{EW}$ variation of CIV BAL troughs is about half that of Si IV troughs. See Section 4.6 .

10. Our large sample clearly establishes that when a BAL quasar shows multiple troughs of the same ion, these troughs usually strengthen or weaken together. We explore this phenomenon for $\mathrm{C}$ IV, finding that $\approx 78 \%$ of high-velocity troughs vary in a coordinated manner with the lowestvelocity trough. Coordinated trough variations are found even for velocity offsets as large as $15000-20000 \mathrm{~km} \mathrm{~s}^{-1}$. See Section 4.7.

11. We have examined if BAL EW variations on several timescales depend upon quasar properties, including $L_{\mathrm{Bol}}, L_{\mathrm{Bol}} / L_{\mathrm{Edd}}, M_{\mathrm{BH}}$, redshift, and radio loudness. Within the ranges of these properties spanned by our sample, we do not find any strong dependences. There may be a hint that C IV EW variations depend on $L_{\text {Bol }}$ and/or $L_{\text {Bol }} / L_{\text {Edd }}$. See Section 4.8.

Considering the observational results above, we discuss implications for quasar outflows. The main implications are the following:

1. The high observed frequency of BAL variability on multi-year timescales is generally supportive of models where most BAL absorption arises at radii of 10-1000 light days. However, a significant minority of BAL troughs are remarkably stable and may be associated with absorption on larger scales. See Section 5.1.

2. Our measurements of EW variations as a function of timescale can be used to infer an (EWdependent) average lifetime for a BAL trough along our line-of-sight of $\left\langle t_{\mathrm{BAL}}\right\rangle=1600_{-900}^{+2100} \mathrm{yr}$. This average lifetime is long compared to the orbital time of the accretion disk at the wind-launching or BLR radius. See Section 5.2

3. Comparison of the $\Delta \mathrm{EW}$ and $\Delta \mathrm{EW} /\langle\mathrm{EW}\rangle$ distributions with previously identified examples of BAL disappearance indicates that BAL disappearance is an extreme type of general BAL variability, rather than a qualitatively distinct phenomenon. The same appears to apply for BAL emergence. See Section 5.3 .

4. We examine the extent to which a simple onedimensional random-walk model can explain the evolution of BAL-trough EWs. The binomial distribution resulting from this model can acceptably fit the $\triangle \mathrm{EW}$ distribution for $\mathrm{CIV}$ BAL troughs on timescales of $2.0-2.5 \mathrm{yr}$, where we have the best trough statistics. The best-fitting model has a step size of $1.65 \AA$ and six random-walk steps, corresponding to a rest-frame step timescale of $\approx 137$ days. The small derived number of steps is consistent with the non-Gaussian nature of the $\Delta \mathrm{EW}$ distribution, and we derive consistent random-walk parameters when considering the $\triangle \mathrm{EW}$ distribution for Si IV troughs. A simulation of randomly walking trough EWs shows the bestfitting model derived for timescales of 2.0-2.5 yr also acceptably represents EW variations over a wider range of timescales. See Section 5.4.

5. Coordinated trough variability for BAL quasars showing multiple troughs implies the existence of 
a coordinating mechanism capable of affecting outflow streamlines spread over a wide range of radii. The mechanism may be changes in the shielding gas that lead to changes in the level of EUV/X-ray radiation reaching the streamlines. Such changes in shielding gas may be a driver of BAL variability more generally. See Section 5.5

The ongoing BOSS ancillary project on quasar-wind variability continues to enlarge the sample of BAL quasars with high-quality spectra spanning multiple years, and we hope to obtain an additional 1-2 epochs of observation for the associated BAL quasars as part of the Time Domain Spectroscopic Survey (TDSS) of the SDSS-IV 22 These data should enable multiple further studies of BAL variability, and here we highlight a few selected ways that the main results found above might be advanced. First, in this paper we have focused upon variability of the relatively high-ionization transitions of CIV and Si IV, and it would be worthwhile to extend large-sample analyses to other transitions including MgII, Al III, Fe II, and Fe III (e.g., Hall et al. 2011; Vivek et al. 2012). The outflow structure along the lineof-sight may differ significantly when such lower ionization transitions are present. Second, additional data can improve constraints upon the fraction of BALs showing variability, BAL lifetimes, and our simple random-walk model for BAL EW variability. Ideally, one would also like to test our measurements of BAL variability against specific predictions made by advancing models of quasar winds. Third, our results on coordinated trough variability for BAL quasars showing multiple troughs indicate that large-scale investigations of BAL vs. continuum variability are important to pursue. Continuum variability investigations in the rest-frame UV should be possible once the BOSS spectrophotometric calibration is improved, and limited X-ray continuum monitoring should be possible with targeted observations and the eROSITA all-sky survey (e.g., Merloni et al. 2012). Fourth, a largescale investigation of BAL vs. emission-line variability should give unique insights into the quasar-wind contribution to high-ionization line production. Finally, the full utilization (see Section [3.2) and gathering of additional observational epochs will allow assessment of how various types of BAL troughs evolve, and perhaps coevolve, over timescales of days-to-years. Additional epochs will also allow a large-scale search for BAL acceleration events which have generally proved elusive to date (e.g., Gibson et al. 2008, 2010; Capellupo et al. 2012).

We gratefully acknowledge financial support from National Science Foundation grant AST-1108604 (NFA, WNB, DPS) and from NSERC (PBH). We thank K. Dawson, M. Eracleous, and D. Schlegel for helpful discussions. We also thank the anonymous referee for constructive feedback.

Funding for SDSS-III has been provided by the Alfred P. Sloan Foundation, the Participating Institutions, the National Science Foundation, and the U.S. Department of Energy Office of Science. The SDSS-III web site is http://www.sdss3.org/

SDSS-1II is managed by the Astrophysical Research Consortium for the Participating Institutions of the SDSS-III Collaboration including the University of Arizona, the Brazilian Participation Group, Brookhaven National Laboratory, University of Cambridge, Carnegie Mellon University, University of Florida, the French Participation Group, the German Participation Group, Harvard University, the Instituto de Astrofisica de Canarias, the Michigan State/Notre Dame/JINA Participation Group, Johns Hopkins University, Lawrence Berkeley National Laboratory, Max Planck Institute for Astrophysics, Max Planck Institute for Extraterrestrial Physics, New Mexico State University, New York University, Ohio State University, Pennsylvania State University, University of Portsmouth, Princeton University, the Spanish Participation Group, University of Tokyo, University of Utah, Vanderbilt University, University of Virginia, University of Washington, and Yale University.

\section{REFERENCES}

Abazajian, K. N., Adelman-McCarthy, J. K., Agüeros, M. A., et al. 2009, ApJS, 182, 543

Allen, J. T., Hewett, P. C., Maddox, N., Richards, G. T., \& Belokurov, V. 2011, MNRAS, 410, 860

Arav, N., Becker, R. H., Laurent-Muehleisen, S. A., et al. 1999, ApJ, 524, 566

Arav, N., Borguet, B., Chamberlain, C., Edmonds, D., \& Danforth, C. 2013, ArXiv e-prints, arXiv:1305.2181

Barlow, T. A. 1993, PhD thesis, UCSD

Barlow, T. A., Junkkarinen, V. T., Burbidge, E. M., et al. 1992, ApJ, 397, 81

Baskin, A., Laor, A., \& Hamann, F. 2013, MNRAS, 432, 1525

Becker, R. H., White, R. L., Gregg, M. D., et al. 2000, ApJ, 538, 72

Becker, R. H., White, R. L., \& Helfand, D. J. 1995, ApJ, 450, 559

Bentz, M. C., Peterson, B. M., Netzer, H., Pogge, R. W., \& Vestergaard, M. 2009, ApJ, 697, 160

Borguet, B. C. J., Arav, N., Edmonds, D., Chamberlain, C., \& Benn, C. 2013, ApJ, 762, 49

Busca, N. G., Delubac, T., Rich, J., et al. 2013, A\&A, 552, A96

Capellupo, D. M., Hamann, F., Shields, J. C., Halpern, J. P., \& Barlow, T. A. 2013, MNRAS, 429, 1872

22 The current planning for SDSS-IV is briefly described at http://www.sdss3.org/future/
Capellupo, D. M., Hamann, F., Shields, J. C., Rodríguez Hidalgo, P., \& Barlow, T. A. 2011, MNRAS, 413, 908

一. 2012, MNRAS, 422, 3249

Chartas, G., Saez, C., Brandt, W. N., Giustini, M., \& Garmire, G. P. 2009, ApJ, 706, 644

Collin, S., Kawaguchi, T., Peterson, B. M., \& Vestergaard, M. 2006, A\&A, 456, 75

Dawson, K. S., Schlegel, D. J., Ahn, C. P., et al. 2013, AJ, 145, 10

Di Matteo, T., Springel, V., \& Hernquist, L. 2005, Nature, 433, 604

Eisenstein, D. J., Weinberg, D. H., Agol, E., et al. 2011, AJ, 142, 72

Emmering, R. T., Blandford, R. D., \& Shlosman, I. 1992, ApJ, 385,460

Faucher-Giguère, C.-A., Quataert, E., \& Murray, N. 2012, MNRAS, 420, 1347

Filiz Ak, N., Brandt, W. N., Hall, P. B., et al. 2012, ApJ, 757, 114

Gallagher, S. C., Brandt, W. N., Chartas, G., \& Garmire, G. P. 2002, ApJ, 567, 37

Gallagher, S. C., Brandt, W. N., Chartas, G., et al. 2006, ApJ, 644, 709

Gallagher, S. C., Brandt, W. N., Wills, B. J., et al. 2004, ApJ, 603, 425

Ganguly, R., \& Brotherton, M. S. 2008, ApJ, 672, 102 
Ganguly, R., Brotherton, M. S., Cales, S., et al. 2007, ApJ, 665, 990

Gehrels, N. 1986, ApJ, 303, 336

Gibson, R. R., Brandt, W. N., Gallagher, S. C., Hewett, P. C., \& Schneider, D. P. 2010, ApJ, 713, 220

Gibson, R. R., Brandt, W. N., Schneider, D. P., \& Gallagher, S. C. 2008, ApJ, 675, 985

Gibson, R. R., Jiang, L., Brandt, W. N., et al. 2009, ApJ, 692, 758

Gunn, J. E., Carr, M., Rockosi, C., et al. 1998, AJ, 116, 3040

Gunn, J. E., Siegmund, W. A., Mannery, E. J., et al. 2006, AJ, 131,2332

Haggard, D., Arraki, K. S., Green, P. J., Aldcroft, T., \& Anderson, S. F. 2012, in Astronomical Society of the Pacific Conference Series, Vol. 460, AGN Winds in Charleston, ed. G. Chartas, F. Hamann, \& K. M. Leighly, 98

Hall, P. B., Anosov, K., White, R. L., et al. 2011, MNRAS, 411, 2653

Hamann, F. 1998, ApJ, 500, 798

Hamann, F., \& Sabra, B. 2004, in Astronomical Society of the Pacific Conference Series, Vol. 311, AGN Physics with the Sloan Digital Sky Survey, ed. G. T. Richards \& P. B. Hall, 203

Hamann, F. W., Netzer, H., \& Shields, J. C. 2000, ApJ, 536, 101

Hewett, P. C., \& Wild, V. 2010, MNRAS, 405, 2302

Kaspi, S., Brandt, W. N., Maoz, D., et al. 2007, ApJ, 659, 997

Kaspi, S., Maoz, D., Netzer, H., et al. 2005, ApJ, 629, 61

Kaspi, S., Brandt, W. N., George, I. M., et al. 2002, ApJ, 574, 643

Kelly, B. C. 2007, ApJ, 665, 1489

Konigl, A., \& Kartje, J. F. 1994, ApJ, 434, 446

Laor, A., \& Brandt, W. N. 2002, ApJ, 569, 641

Leighly, K. M. 2004, ApJ, 611, 125

Lilliefors, H. 1967, Journal of the American Statistical Association, 62, 399

Lundgren, B. F., Wilhite, B. C., Brunner, R. J., et al. 2007, ApJ, 656, 73

Margala, D., \& Kirkby, D. 2011, in SDSS BOSS QSO Workshop, Princeton, NJ, USA

Merloni, A., Predehl, P., Becker, W., et al. 2012, ArXiv e-prints, arXiv:1209.3114

Miller, B. P., Brandt, W. N., Gibson, R. R., Garmire, G. P., \& Shemmer, O. 2009, ApJ, 702, 911

Miller, B. P., Welling, C. A., Brandt, W. N., \& Gibson, R. R. 2012, in Astronomical Society of the Pacific Conference Series, Vol. 460, AGN Winds in Charleston, ed. G. Chartas, F. Hamann, \& K. M. Leighly, 118

Misawa, T., Eracleous, M., Charlton, J. C., \& Kashikawa, N. 2007, ApJ, 660, 152

Murray, N., Chiang, J., Grossman, S. A., \& Voit, G. M. 1995, ApJ, 451, 498

Pâris, I., Petitjean, P., Aubourg, É., et al. 2012, A\&A, 548, A66 -. 2013, In press

Press, W. H., Teukolsky, S. A., Vetterling, W. T., \& Flannery,

B. P. 1992, Numerical recipes. The art of scientific computing

Proga, D. 2000, ApJ, 538, 684

Proga, D., \& Kallman, T. R. 2004, ApJ, 616, 688
Randles, R. H., Fligner, M. A., Policello, G. E., \& Wolfe, D. A. 1980, Journal of American Statistic Association, 75, 168

Richards, G. T., Fan, X., Newberg, H. J., et al. 2002, AJ, 123, 2945

Richards, G. T., Kruczek, N. E., Gallagher, S. C., et al. 2011, AJ, 141,167

Ross, N. P., Myers, A. D., Sheldon, E. S., et al. 2012, ApJS, 199, 3

Rupke, D. S. N., \& Veilleux, S. 2011, ApJ, 729, L27

Saez, C., Brandt, W. N., Gallagher, S. C., Bauer, F. E., \& Garmire, G. P. 2012, ApJ, 759, 42

Schlegel, D. J., Finkbeiner, D. P., \& Davis, M. 1998, ApJ, 500, 525

Schneider, D. P., Hall, P. B., Richards, G. T., et al. 2007, AJ, 134,102

Schneider, D. P., Richards, G. T., Hall, P. B., et al. 2010, AJ, 139, 2360

Shankar, F., Dai, X., \& Sivakoff, G. R. 2008, ApJ, 687, 859

Shen, Y. 2013, Bulletin of the Astronomical Society of India, 41, 61

Shen, Y., Greene, J. E., Strauss, M. A., Richards, G. T., \& Schneider, D. P. 2008, ApJ, 680, 169

Shen, Y., Richards, G. T., Strauss, M. A., et al. 2011, ApJS, 194, 45

Sim, S. A., Proga, D., Kurosawa, R., et al. 2012, MNRAS, 426, 2859

Sim, S. A., Proga, D., Miller, L., Long, K. S., \& Turner, T. J. 2010, MNRAS, 408, 1396

Slosar, A., Iršič, V., Kirkby, D., et al. 2013, Journal of Cosmology and Astroparticle Physics, 4, 26

Smee, S. A., Gunn, J. E., Uomoto, A., et al. 2013, AJ, 146, 32

Smith, L. J., \& Penston, M. V. 1988, MNRAS, 235, 551

Sturm, E., González-Alfonso, E., Veilleux, S., et al. 2011, ApJ, 733, L16

Suzuki, N. 2013, in SDSS-III Collaboration Meeting, Baltimore, MD, USA

Trump, J. R., Hall, P. B., Reichard, T. A., et al. 2006, ApJS, 165,

Turnshek, D. A. 1988, in QSO Absorption Lines: Probing the Universe, ed. J. C. Blades, D. A. Turnshek, \& C. A. Norman, 17

Turnshek, D. A., Grillmair, C. J., Foltz, C. B., \& Weymann, R. J. 1988, ApJ, 325, 651

Vanden Berk, D. E., Richards, G. T., Bauer, A., et al. 2001, AJ, 122,549

Vanden Berk, D. E., Wilhite, B. C., Kron, R. G., et al. 2004, ApJ, 601, 692

Vilkoviskij, E. Y., \& Irwin, M. J. 2001, MNRAS, 321, 4

Vivek, M., Srianand, R., Petitjean, P., et al. 2012, MNRAS, 423 , 2879

Welling, C. A., Miller, B. P., Brandt, W. N., Capellupo, D. M., \& Gibson, R. R. 2013, MNRAS, submitted

Weymann, R. J., Carswell, R. F., \& Smith, M. G. 1981, ARA\&A, 19,41

Weymann, R. J., Morris, S. L., Foltz, C. B., \& Hewett, P. C. 1991, ApJ, 373, 23

York, D. G., Adelman, J., Anderson, Jr., J. E., et al. 2000, AJ, 120,1579 
TABLE 1

Sample-Based Studies of BAL Quasar Variability

\begin{tabular}{|c|c|c|c|}
\hline Reference & \# of Quasars & $\begin{array}{c}\Delta t \text { Range } \\
(\mathrm{yr})\end{array}$ & \# of Epochs \\
\hline Barlow (1993) & 23 & $0.2-1.2$ & $2-6$ \\
\hline Lundgren et al. (2007) & 29 & $0.05-0.3$ & 2 \\
\hline Gibson et al. (2008) & 13 & $3.0-6.1$ & 2 \\
\hline Gibson et al. (2010) & 14 & $0.04-6.8$ & $2-4$ \\
\hline Capellupo et al. $(2011,2012,2013)$ & 24 & $0.02-8.7$ & $2-13$ \\
\hline Vivek et al. (2012) & 5 & $0.01-5$ & $4-14$ \\
\hline Haggard et al. (2012) & 17 & $0.001-0.9$ & 6 \\
\hline Filiz Ak et al. $(2012)^{\mathrm{b}}$ & 19 & $1.1-3.9$ & $2-4$ \\
\hline Welling et al. $(2013)^{\mathrm{c}}$ & 46 & $0.2-16.4$ & $2-6$ \\
\hline This study & 291 & $0.0006-3.7$ & $2-12$ \\
\hline Full BOSS Ancillary & 2105 & $0.0006-6$ & $2-12$ \\
\hline
\end{tabular}

${ }^{a}$ Fe low-ionization BAL quasars

b Quasars with disappearing BAL troughs

c Radio-loud BAL quasars 
TABLE 2

Civ BAL Troughs

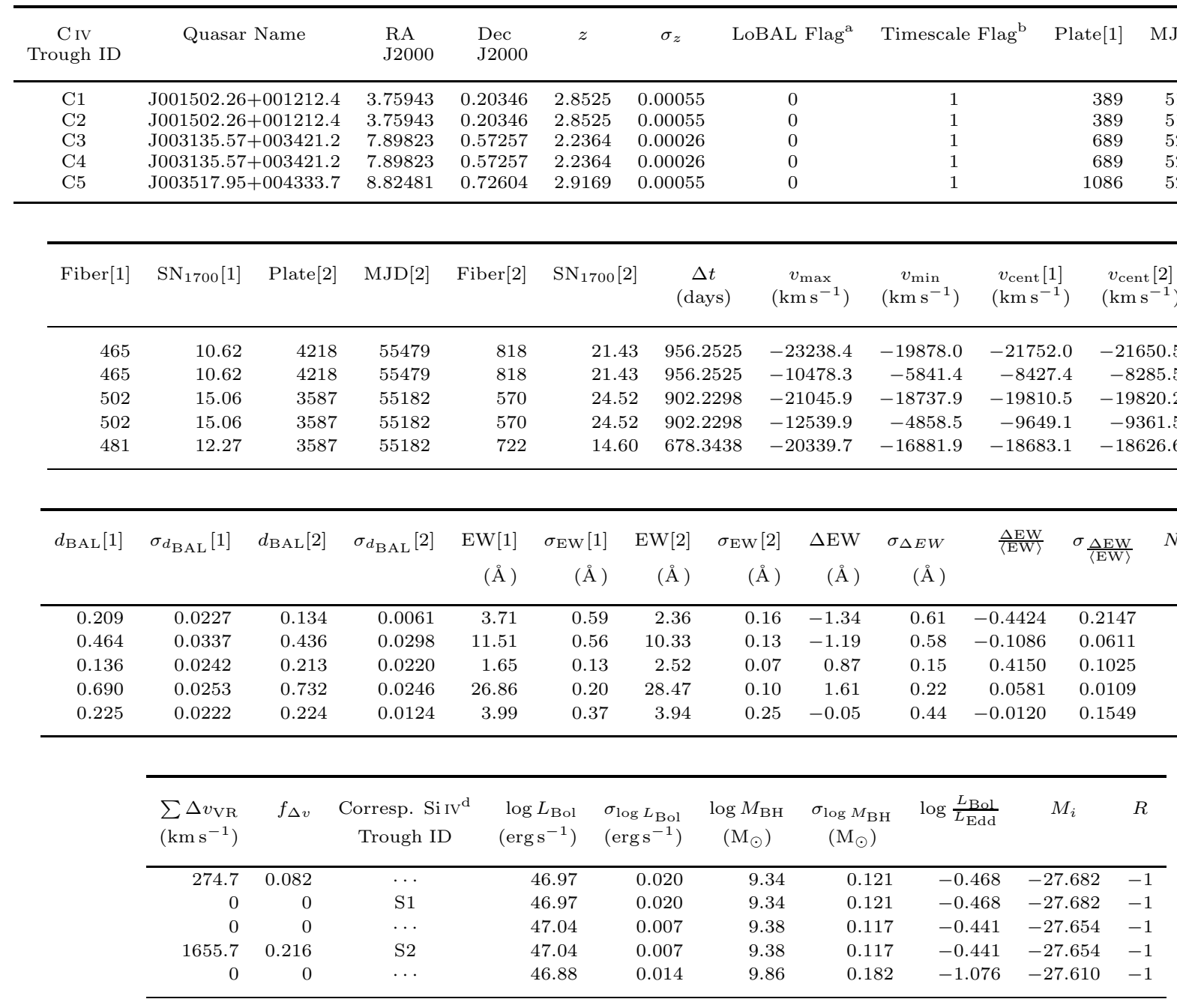

Note. - (This table is available in its entirety in a machine-readable form in the online journal. A portion is shown here for guidance regarding its form and content.)

Note. - Throughout this table [1] indicates the first-epoch spectra and [2] indicates the second-epoch spectra.

a 1 for low-ionization BAL quasars, 0 otherwise

${ }^{\mathrm{b}} 1$ for timescales of more than $1 \mathrm{yr}\left(\Delta t_{\min , 1}\right)$, 0 otherwise $\left(\Delta t_{\min }\right)$

${ }^{\mathrm{c}}$ Number of variable regions found in the BAL trough

${ }^{\mathrm{d}}$ Corresponding Si IV BAL trough ID as given in Table 3 
TABLE 3

Si IV BAL Troughs

\begin{tabular}{|c|c|c|c|c|c|c|c|c|c|}
\hline $\begin{array}{c}\text { Si IV } \\
\text { Trough ID }\end{array}$ & Quasar Name & $\begin{array}{l}\text { RA } \\
\text { J2000 }\end{array}$ & $\begin{array}{l}\text { Dec } \\
\text { J2000 }\end{array}$ & $z$ & $\sigma_{z}$ & LoBAL Flag ${ }^{\mathrm{a}}$ & Timescale Flag ${ }^{\mathrm{b}}$ & Plate[1] & MJD[1] \\
\hline S1 & J001502.26+001212.4 & 3.75943 & 0.20346 & 2.8525 & 0.00055 & 0 & 1 & 389 & 51795 \\
\hline S2 & J003135.57+003421.2 & 7.89821 & 0.57260 & 2.2364 & 0.00026 & 0 & 1 & 689 & 52262 \\
\hline S3 & J003517.95+004333.7 & 8.82481 & 0.72604 & 2.9169 & 0.00055 & 0 & 1 & 1086 & 52525 \\
\hline $\mathrm{S} 4$ & J004613.54+010425.7 & 11.55643 & 1.07381 & 2.1492 & 0.00028 & 0 & 1 & 691 & 52199 \\
\hline S5 & J005419.99+002727.9 & 13.58329 & 0.45776 & 2.5143 & 0.00025 & 0 & 1 & 394 & 51913 \\
\hline
\end{tabular}

\begin{tabular}{|c|c|c|c|c|c|c|c|c|c|c|c|c|c|}
\hline Fiber [1] & $\mathrm{SN}_{1700}[1]$ & Plate[2] & MJD[2] & Fiber[2] & $\mathrm{SN}_{1700}[2]$ & \multicolumn{2}{|c|}{$\begin{array}{c}\Delta t \\
\text { (days) }\end{array}$} & $\begin{array}{c}v_{\max } \\
\left(\mathrm{km} \mathrm{s}^{-1}\right)\end{array}$ & $\begin{array}{c}v_{\min } \\
\left(\mathrm{km} \mathrm{s}^{-1}\right)\end{array}$ & $\begin{array}{c}v_{\text {cent }}[1] \\
\left(\mathrm{km} \mathrm{s}^{-1}\right)\end{array}$ & \multicolumn{2}{|c|}{$\begin{array}{c}v_{\text {cent }}[2] \\
\left(\mathrm{km} \mathrm{s}^{-1}\right)\end{array}$} & . \\
\hline 465 & 10.62 & 4218 & 55479 & 818 & 21.43 & \multicolumn{2}{|c|}{956.2525} & -12074.1 & -5872.0 & -8972.6 & \multicolumn{2}{|c|}{-8908.4} & \\
\hline 502 & 15.06 & 3587 & 55182 & 570 & 24.52 & \multicolumn{2}{|c|}{902.2298} & -12521.9 & -4933.6 & -8905.7 & \multicolumn{2}{|c|}{-8831.1} & \\
\hline 481 & 12.27 & 3587 & 55182 & 722 & 14.60 & \multicolumn{2}{|c|}{678.3438} & -5224.5 & -3000.0 & -4286.6 & \multicolumn{2}{|c|}{-4296.7} & \\
\hline 460 & 23.70 & 3589 & 55186 & 866 & 28.94 & \multicolumn{2}{|c|}{948.4810} & -20051.0 & -9612.2 & -14741.9 & \multicolumn{2}{|c|}{-14841.3} & \\
\hline 511 & 31.12 & 4224 & 55481 & 726 & 25.32 & \multicolumn{2}{|c|}{1015.2949} & -11686.2 & -5045.8 & -8284.2 & \multicolumn{2}{|c|}{-8293.7} & \\
\hline \multirow[t]{2}{*}{$d_{\mathrm{BAL}}[1]$} & \multirow[t]{2}{*}{$\sigma_{d_{\mathrm{BAL}}}[1]$} & \multirow[t]{2}{*}{$d_{\mathrm{BAL}}[2]$} & \multirow[t]{2}{*}{$\sigma_{d_{\mathrm{BAL}}}[2]$} & $\mathrm{EW}[1]$ & $\sigma_{\mathrm{EW}}[1]$ & \multirow{2}{*}{$\begin{array}{r}\mathrm{EW}[2] \\
(\AA)\end{array}$} & \multirow{2}{*}{$\begin{array}{r}\sigma_{\mathrm{EW}}[2] \\
(\AA)\end{array}$} & \multirow{2}{*}{$\begin{array}{r}\Delta \mathrm{EW} \\
(\AA)\end{array}$} & $\sigma_{\Delta E W}$ & \multirow[t]{2}{*}{$\frac{\Delta \mathrm{EW}}{\langle\mathrm{EW}\rangle}$} & \multirow{2}{*}{$\sigma_{\frac{\Delta \mathrm{EW}}{\langle\mathrm{EW}\rangle}}$} & \multirow[t]{2}{*}{$N_{\mathrm{VR}}{ }^{\mathrm{c}}$} & $\cdots$ \\
\hline & & & & $(\AA)$ & $(\AA)$ & & & & $(\AA)$ & & & & \\
\hline 0.221 & 0.0159 & 0.139 & 0.0059 & 6.41 & 0.68 & 3.97 & 0.12 & -2.44 & 0.69 & -0.4707 & 0.1292 & 1 & \\
\hline 0.311 & 0.0158 & 0.361 & 0.0175 & 10.84 & 0.24 & 12.60 & 0.12 & 1.76 & 0.27 & 0.1499 & 0.0315 & 1 & \\
\hline 0.439 & 0.0456 & 0.432 & 0.0437 & 4.76 & 0.21 & 4.69 & 0.13 & -0.07 & 0.25 & -0.0144 & 0.0731 & 0 & \\
\hline 0.223 & 0.0058 & 0.171 & 0.0082 & 10.46 & 0.29 & 8.16 & 0.17 & -2.30 & 0.34 & -0.2467 & 0.0484 & 3 & \\
\hline 0.200 & 0.0087 & 0.174 & 0.0107 & 6.11 & 0.16 & 5.33 & 0.12 & -0.78 & 0.20 & -0.1371 & 0.0475 & 3 & \\
\hline
\end{tabular}

\begin{tabular}{rrrrrrrrrr}
\hline $\begin{array}{r}\sum \Delta v_{\mathrm{VR}} \\
\left(\mathrm{km} \mathrm{s}^{-1}\right)\end{array}$ & $f_{\Delta v}$ & $\begin{array}{c}\text { Corresp. C IV } \\
\text { Trough ID }\end{array}$ & $\begin{array}{c}\log L_{\mathrm{Bol}} \\
\left(\mathrm{erg} \mathrm{s}^{-1}\right)\end{array}$ & $\begin{array}{c}\sigma_{\log L_{\mathrm{Bol}}} \\
\left(\mathrm{erg} \mathrm{s}^{-1}\right)\end{array}$ & $\begin{array}{c}\log M_{\mathrm{BH}} \\
\left(\mathrm{M}_{\odot}\right)\end{array}$ & $\begin{array}{c}\sigma_{\log M_{\mathrm{BH}}} \\
\left(\mathrm{M}_{\odot}\right)\end{array}$ & $\log \frac{L_{\mathrm{Bol}}}{L_{\mathrm{Edd}}}$ & $M_{i}$ & $R$ \\
\hline 276.1 & 0.045 & $\mathrm{C} 2$ & 46.97 & 0.020 & 9.342 & 0.121 & -0.468 & -27.682 & -1 \\
345.1 & 0.046 & $\mathrm{C} 4$ & 47.04 & 0.007 & 9.380 & 0.117 & -0.441 & -27.654 & -1 \\
0 & 0 & $\ldots$ & 46.88 & 0.014 & 9.855 & 0.182 & -1.076 & -27.610 & -1 \\
1858.4 & 0.178 & $\ldots$ & 47.08 & 0.009 & 9.220 & 0.220 & -0.245 & -28.404 & 11.4 \\
1311.2 & 0.198 & $\mathrm{C} 10$ & 47.07 & 0.005 & 9.652 & 0.043 & -0.681 & -28.209 & -1 \\
\hline
\end{tabular}

Note. - (This table is available in its entirety in a machine-readable form in the online journal. A portion is shown here for guidance regarding its form and content.)

NoтE. - Throughout this table [1] indicates the first-epoch spectra and [2] indicates the second-epoch spectra.

${ }^{a} 1$ for low-ionization BAL quasars, 0 otherwise

$\mathrm{b}_{1}$ for timescales of more than $1 \mathrm{yr}\left(\Delta t_{\min , 1}\right), 0$ otherwise $\left(\Delta t_{\min }\right)$

${ }^{\mathrm{c}}$ Number of variable regions found in the BAL trough

${ }^{\mathrm{d}}$ Corresponding $\mathrm{C}$ IV BAL trough ID as given in Table 2 As explained in Section 4.6, we search for accompanying C IV BAL troughs for $\mathrm{Si}$ IV troughs with $-3000>v_{\text {cent }}>-13000 \mathrm{~km} \mathrm{~s}^{-1}$. We found that five Si IV troughs, S3, S13, S201, S202, and S237, have an accompanying $\mathrm{C}$ IV trough that does not satisfy our BAL-trough identification criteria. 
TABLE 4

Civ BAL-Trough Variable Regions

\begin{tabular}{cccccccccrr}
\hline $\begin{array}{c}\text { C IV } \\
\text { VR ID }\end{array}$ & Quasar Name & MJD[1] & MJD[2] & $\begin{array}{c}\mathrm{CIV}_{\mathrm{IV}}^{\mathrm{a}} \\
\text { Trough ID }\end{array}$ & $\begin{array}{c}v_{\max } \\
\left(\mathrm{km} \mathrm{s}^{-1}\right)\end{array}$ & $\begin{array}{c}v_{\min } \\
\left(\mathrm{km} \mathrm{s}^{-1}\right)\end{array}$ & $\begin{array}{r}v_{\max , \mathrm{VR}} \\
\left(\mathrm{km} \mathrm{s}^{-1}\right)\end{array}$ & $\begin{array}{c}v_{\min , \mathrm{VR}} \\
\left(\mathrm{km} \mathrm{s}^{-1}\right)\end{array}$ & $\begin{array}{c}\Delta v_{\mathrm{VR}} \\
\left(\mathrm{km} \mathrm{s}^{-1}\right)\end{array}$ & \multicolumn{1}{c}{$v_{\mathrm{nrt}}$} \\
\hline CV1 & J001502.26+001212.4 & 51795 & 55479 & $\mathrm{C} 1$ & -23238.4 & -19878.0 & -23160.0 & -22885.4 & 274.7 & -0.85 \\
CV2 & J003135.57+003421.2 & 52262 & 55182 & $\mathrm{C} 4$ & -12539.9 & -4858.5 & -11608.3 & -10642.5 & 965.8 & -0.51 \\
CV3 & J003135.57+003421.2 & 52262 & 55182 & $\mathrm{C} 4$ & -12539.9 & -4858.5 & -10504.5 & -9814.5 & 690.0 & -0.18 \\
CV4 & J004323.43-001552.4 & 52261 & 55184 & $\mathrm{C} 6$ & -21405.5 & -10137.6 & -16564.3 & -15186.6 & 1377.7 & 0 \\
CV5 & J004323.43-001552.4 & 52261 & 55184 & $\mathrm{C} 6$ & -21405.5 & -10137.6 & -15048.8 & -12153.5 & 2895.3 & 0.40 \\
\hline
\end{tabular}

Note. - (This table is available in its entirety in a machine-readable form in the online journal. A portion is shown here for guidance regarding its form and content.)

Note. - Throughout this table [1] indicates the first-epoch spectra and [2] indicates the second-epoch spectra.

${ }^{a}$ BAL trough IDs as given in Table 2

TABLE 5

Si iv BAL-Trough Variable Regions

\begin{tabular}{|c|c|c|c|c|c|c|c|c|c|c|}
\hline $\begin{array}{l}\text { Si IV } \\
\text { VR ID }\end{array}$ & Quasar Name & $\mathrm{MJD}[1]$ & $\mathrm{MJD}[2]$ & $\begin{array}{c}\mathrm{Si}_{\mathrm{IV}}^{\mathrm{a}} \\
\text { Trough ID }\end{array}$ & $\begin{array}{c}v_{\max } \\
\left(\mathrm{km} \mathrm{s}^{-1}\right)\end{array}$ & $\begin{array}{c}v_{\min } \\
\left(\mathrm{km} \mathrm{s}^{-1}\right)\end{array}$ & $\begin{array}{l}v_{\max , \mathrm{VR}} \\
\left(\mathrm{km} \mathrm{s}^{-1}\right)\end{array}$ & $\begin{array}{c}v_{\min , \mathrm{VR}} \\
\left(\mathrm{km} \mathrm{s}^{-1}\right)\end{array}$ & $\begin{array}{c}\Delta v_{\mathrm{VR}} \\
\left(\mathrm{km} \mathrm{s}^{-1}\right)\end{array}$ & $v_{\text {nrt }}$ \\
\hline SV1 & J001502.26+001212.4 & 51795 & 55479 & S1 & -12074.1 & -5872.0 & -8365.8 & -8089.7 & 276.1 & -0.24 \\
\hline SV2 & J003135.57+003421.2 & 52262 & 55182 & S2 & -12521.9 & -4933.6 & -9137.9 & -8792.8 & 345.1 & 0.02 \\
\hline SV3 & J004613.54+010425.7 & 52199 & 55186 & $\mathrm{~S} 4$ & -20051.0 & -9612.2 & -19915.7 & -18952.7 & 963.0 & 0.91 \\
\hline SV4 & J004613.54+010425.7 & 52199 & 55186 & $\mathrm{~S} 4$ & -20051.0 & -9612.2 & -18126.9 & -17507.4 & 619.5 & 0.60 \\
\hline SV5 & J004613.54+010425.7 & 52199 & 55186 & $\mathrm{~S} 4$ & -20051.0 & -9612.2 & -11649.9 & -11374.0 & 275.9 & -0.61 \\
\hline
\end{tabular}

Note. - (This table is available in its entirety in a machine-readable form in the online journal. A portion is shown here for guidance regarding its form and content.)

Note. - Throughout this table [1] indicates the first-epoch spectra and [2] indicates the second-epoch spectra.

${ }^{a}$ BAL trough IDs as given in Table 3

TABLE 6

VARIABILITY COMPARISON BETWEen THE LOWEST

Velocity BAL Trough and AdDitional BAL

TROUGHS

\begin{tabular}{lcc}
\hline \hline & \multicolumn{2}{c}{ Number of Additional C IV Troughs } \\
\cline { 2 - 3 } & $\Delta E W<0$ & $\Delta E W>0$ \\
\hline$\Delta \mathrm{EW}_{\text {low vel }}<0$ & 57 & 13 \\
$\Delta \mathrm{EW}_{\text {low vel }}>0$ & 17 & 50 \\
\hline
\end{tabular}


TABLE 7

Spearman Rank Correlation Test Probabilities for Quasar Properties vs. BAL-Trough EW VARiations

\begin{tabular}{|c|c|c|c|c|c|c|}
\hline & \multicolumn{3}{|c|}{ C IV } & \multicolumn{3}{|c|}{ Si IV } \\
\hline & $<1 \mathrm{yr}$ & $1-2.5 \mathrm{yr}$ & $>2.5 \mathrm{yr}$ & $<1 \mathrm{yr}$ & $1-2.5 \mathrm{yr}$ & $>2.5 \mathrm{yr}$ \\
\hline \multicolumn{7}{|c|}{$L_{\mathrm{Bol}}$} \\
\hline$|\Delta E W|$ & 78.4 & 0.2 & 5.2 & 65.3 & 5.5 & 79.8 \\
\hline$\left|\frac{\Delta E W}{\langle E W\rangle}\right|$ & 31.5 & 83.2 & 67.1 & 44.3 & 90.6 & 11.1 \\
\hline \multicolumn{7}{|c|}{$L_{\mathrm{Bol}} / L_{\mathrm{Edd}}$} \\
\hline$|\Delta E W|$ & 54.6 & 0.1 & 35.1 & 65.6 & 81.5 & 96.2 \\
\hline$\left|\frac{\Delta E W}{\langle E W\rangle}\right|$ & 26.3 & 2.4 & 25.1 & 39.5 & 92.5 & 25.4 \\
\hline \multicolumn{7}{|c|}{$M_{\mathrm{BH}}$} \\
\hline$|\Delta E W|$ & 32.9 & 11.4 & 66.4 & 56.1 & 85.1 & 66.6 \\
\hline$\left|\frac{\Delta E W}{\langle E W\rangle}\right|$ & 45.3 & 1.2 & 32.7 & 38.5 & 85.1 & 7.8 \\
\hline \multicolumn{7}{|c|}{$z$} \\
\hline$|\Delta E W|$ & 70.4 & 1.2 & 12.5 & 49.9 & 31.1 & 6.8 \\
\hline$\left|\frac{\Delta E W}{\langle E W\rangle}\right|$ & 8.4 & 3.4 & 48.3 & 32.9 & 16.9 & 30.9 \\
\hline Number of data points & 82 & 220 & 126 & 56 & 124 & 55 \\
\hline
\end{tabular}

